\title{
Characterization of Gas-Oil Flow in Cyclic Solvent Injection (CSI) for Heavy Oil Recovery
}

\author{
A Thesis \\ Submitted to the Faculty of Graduate Studies and Research \\ In Partial Fulfillment of the Requirements \\ For the Degree of \\ Master of Applied Science \\ in \\ Petroleum Systems Engineering \\ University of Regina
}

By

Sam Yeol Hong

Regina, Saskatchewan

August 3, 2016

Copyright 2016: S. Hong 


\section{UNIVERSITY OF REGINA}

\section{FACULTY OF GRADUATE STUDIES AND RESEARCH SUPERVISORY AND EXAMINING COMMITTEE}

Sam Yeol Hong, candidate for the degree of Master of Applied Science in Petroleum Systems Engineering, has presented a thesis titled, Characterization of Gas-Oil Flow in Cyclic Solvent Injection (CSI) for Heavy Oil Recovery, in an oral examination held on July 28,2016 . The following committee members have found the thesis acceptable in form and content, and that the candidate demonstrated satisfactory knowledge of the subject material.

External Examiner:

Supervisor:

Committee Member:

Committee Member:

Chair of Defense:
Mr. Muhammad Imran, Saskatchewan Research Council

Dr. Fanhua Zeng, Petroleum Systems Engineering

Dr. Yee-Chung Jin, Environmental Systems Engineering

Dr. Ezeddin Shirif, Petroleum Systems Engineering

Dr. Mohamed El-Darieby, Software Systems Engineering 


\section{Abstract}

Cyclic Solvent Injection (CSI) has emerged as an effective post-CHOPS recovery method. It has shown a great potential with the aid of the solvent injection under the huff-n-puff operation. The use of solvent that maintains a strong nature of gas results in the solvent chamber which under the huff-n-puff operation locates at an inner region towards the well. The CSI process is therefore governed by the gas-oil flow as the solvent chamber is dominated by the free gas-oil flow and the heavy oil zone by the dispersed gas-oil flow referred to as the foamy oil flow. The gas-oil flow in CSI eventually appears as the combined flow of free gas and foamy oil across the solvent chamber.

The gas-oil flow in heavy oil systems has been widely investigated based on heavy oil solution gas drive. However, the combined flow of free gas and foamy oil in CSI considerably differs from that in heavy oil solution gas drive and therefore needs to be investigated separately. The differences mainly arise as in CSI the free gas originates from the solvent chamber whereas in heavy oil solution gas drive it evolves from solution gas. Consequently, the combined flow of free gas and foamy oil in CSI yields the characteristics that strongly depend on the pressure depletion rate and the growing solvent chamber.

This study is aimed at characterizing the gas-oil flow in CSI for heavy oil recovery at different pressure depletion rates under the effect of the growing 
solvent chamber. To fulfill the objective, the gas-liquid relative permeability curves are inferred with the use of numerical simulations by history-matching seven lab-scale CSI tests performed at varying pressure depletion rates. The foamy oil behavior is taken into account by applying the modified-fractional flow model. This study therefore not only demonstrates the distinct properties of the gas-oil relative permeability curves in CSI process but also the applicability of the modified-fractional flow model. The sensitivity analysis is performed to examine the phenomena responsible for the distinct behavior of the gas-oil flow in CSI. 


\section{Acknowledgements}

First and foremost, I would like to express my sincere appreciation to my supervisor, Dr. Fanhua Zeng. Dr. Zeng has always been my role model as a professional since the time he taught one of my undergraduate courses at University of Regina. He is the initial person to inspire me to purse my master's degree, and continuously supported and encouraged my work during the master's program. I am truly thankful for his patience and the numerous hours he put in to deliver his knowledge to me.

In addition, I wish to thank all members and fellow students of the Department of Petroleum System Engineering for making my past years so enjoyable. In particular, the individuals of Dr. Zeng's research group have provided great support that I needed. Without their help, it could have been a lot more difficult to complete this work.

Lastly, I would like to thank FGSR for its financial support through International Graduate Student Scholarship, Faculty of Graduate Studies and Research Graduate Scholarship, and Faculty of Graduate Studies and Research Graduate Teaching Assistantship. I am also grateful for the research funding from PTRC. It is their support which enabled this work to happen. 


\section{Post Defense Acknowledgement}

I must thank Mr. Muhammad Imran for the valuable comments and questions that he made as the external examiner for my thesis defense. He has contributed so much to the improvement of my thesis. The value of this work could have been significantly undermined without his contribution. Also, I must mention that the contributions of Dr. Ezeddin Shirif and Dr. Yee-Chung Jin as the committee members were greatly appreciated. 


\section{Dedication}

First of all, I want to thank God for guiding me though the years of my master's program. He always gave me the confidence that I am guided to a righteous way.

I would like to extend thanks to my wife, Sun Hee Bang, and my son, Sangki Hong for all their love and support through this long journey. Sun Hee chose to be away from her parents and other family members in South Korea to support my dream as a professional. Especially, the little boy, Sangki, has been the energy source of my daily life. With all my heart, I promise that I would devote rest of my life to my family and dedicate this work with love to them. 


\section{Table of Contents}

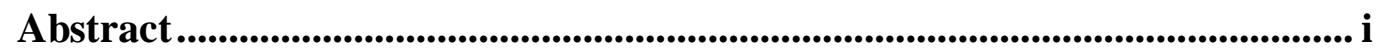

Acknowledgements................................................................................................ iii

Post Defense Acknowledgement .......................................................................... iv

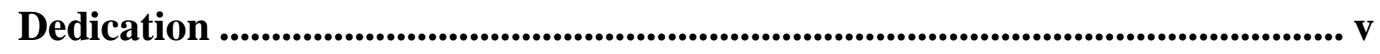

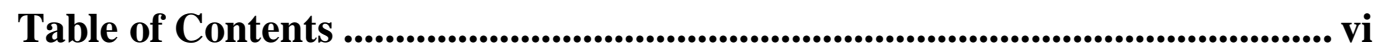

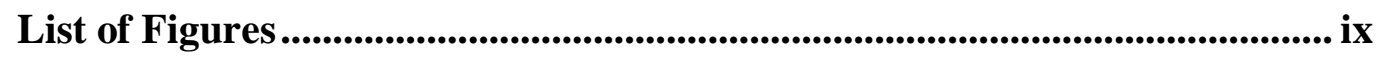

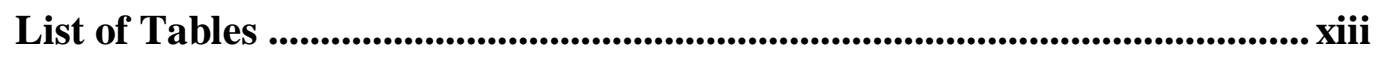

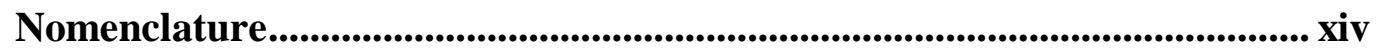

Chapter 1. Introduction ..................................................................................................... 1

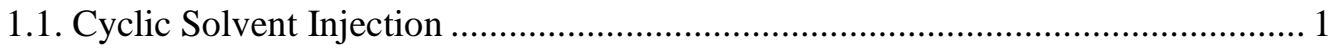

1.2. Field Design for Heavy Oil Recovery …...................................................... 3

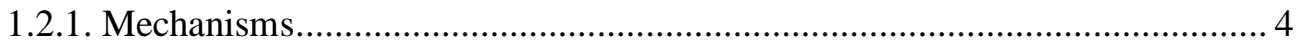

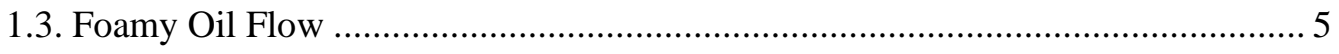

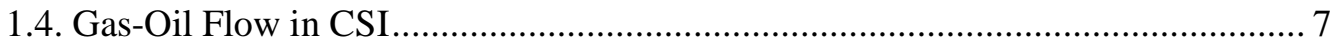

1.5. Gas-Oil Flow in CSI versus in Heavy Oil Solution Gas Drive …........................ 9

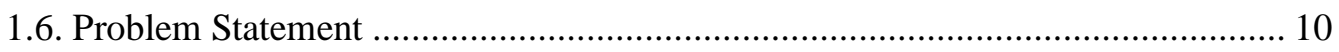

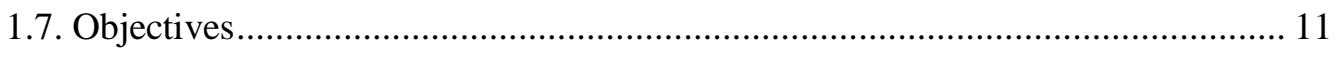

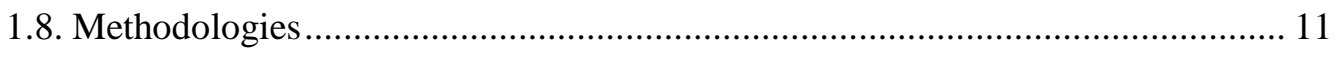

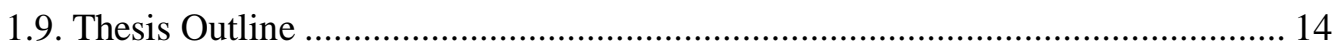

Chapter 2. Literature Review ..................................................................................... 17

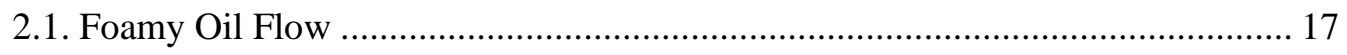

2.2. Gas-Liquid Relative Permeability Curves in Heavy Oil Solution Gas Drive ....... 19

2.3. Gas-Liquid Relative Permeability Curves in CSI .......................................... 21 


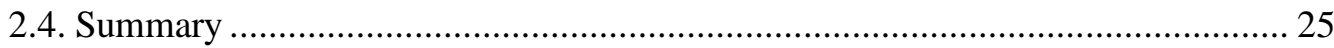

Chapter 3. Experiments .............................................................................. 27

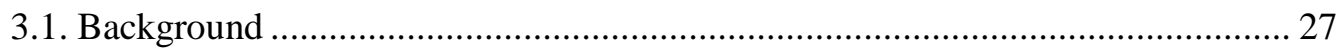

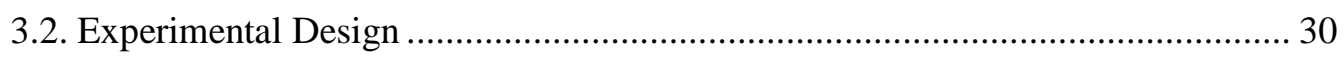

3.2.1. Sandpacking and Sandpack Dimensions ................................................ 30

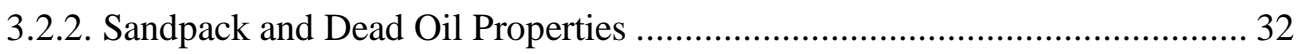

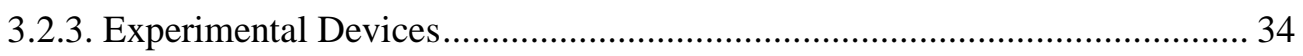

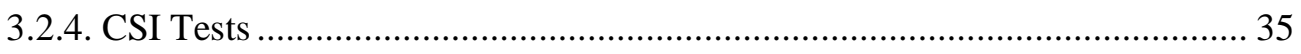

Chapter 4. Numerical Simulations ............................................................................ 38

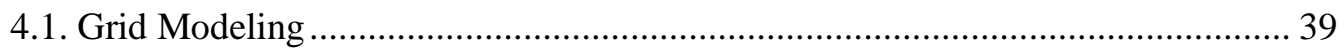

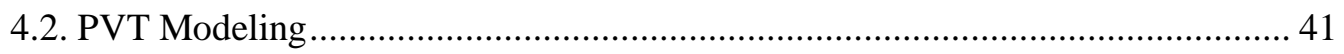

4.3. Capillary Pressure and Dispersion Coefficients ............................................ 44

4.4. Tuning of Gas-Liquid Relative Permeability Curves....................................... 46

Chapter 5. Results ................................................................................................................... 49

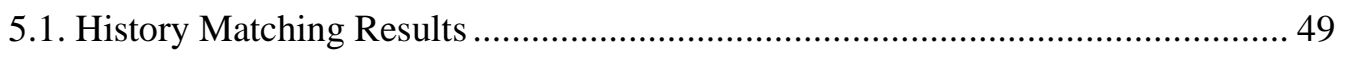

5.2. Tuned Gas-Liquid Relative Permeability Curves ............................................. 58

Chapter 6. Discussions ..................................................................................................... 63

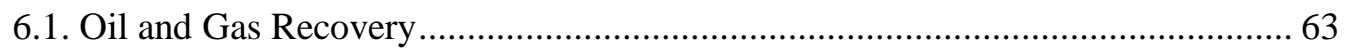

6.2. Gas-Liquid Relative Permeability Curves for Production Stages ...................... 70

6.2.1. Effect of Pressure Depletion Rate ............................................................. 72

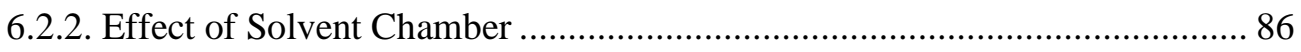

6.3. Gas-Liquid Relative Permeability Curves in Injection Stages ........................... 93

Chapter 7. Conclusions and Future Works .......................................................... 101

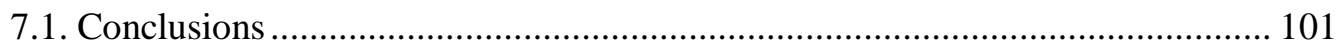

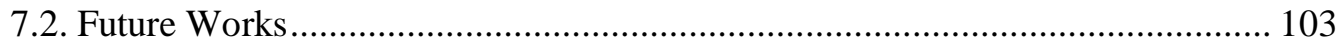


List of References ............................................................................................................. 104

Appendix I: Grid Blocks Coordinates..................................................... A-1

Appendix II: PVT Model ........................................................................ A-10

Appendix III: Gas-Liquid Relative Permeability Tables ........................... A-13 


\section{List of Figures}

Figure 3-1 Diagram of sandpack models with a mimic wormhole at (a) center; (b) top; and (c) bottom 31

Figure 4-1 Grid system with the well (mimic wormhole) located at center....... 40

Figure 4-2 Dead oil viscosity matching at 1 atm 42

Figure 4-3 Dead oil \& propane mixture viscosity matching at $15.4{ }^{\circ} \mathrm{C}$ and $75{ }^{\circ} \mathrm{C}$

Figure 4-4 Capillary pressure curve between gas and oil phases

Figure 5-1 History matching results (a) production profiles (b) model pressure of Test $1(1 \mathrm{kPa} / \mathrm{min}$, center wormhole $)$ 50

Figure 5-2 History matching results (a) production profiles (b) model pressure of Test $2(3 \mathrm{kPa} / \mathrm{min}$, center wormhole) 51

Figure 5-3 History matching results (a) production profiles (b) model pressure of Test $3(5 \mathrm{kPa} / \mathrm{min}$, center wormhole $)$ 52

Figure 5-4 History matching results (a) production profiles (b) model pressure of Test $4(12.5 \mathrm{kPa} / \mathrm{min}$, center wormhole $)$ 53

Figure 5-5 History matching results (a) production profiles (b) model pressure of Test $5(50 \mathrm{kPa} / \mathrm{min}$, center wormhole $)$ 54

Figure 5-6 History matching results (a) production profiles (b) model pressure of Test $6(100 \mathrm{kPa} / \mathrm{min}$, center wormhole $)$ 55 
Figure 5-7 History matching results of Test 7 (500 kPa/5 min, center wormhole)

Figure 5-8 History matching results of Test $8(500 \mathrm{kPa} / 5 \mathrm{~min}$, bottom wormhole)

Figure 5-9 History matching results of Test 9 (500 kPa/5 min, top wormhole)

Figure 5-10 Tuned gas-liquid relative permeability curves of Test $1(1 \mathrm{kPa} / \mathrm{min})$

Figure 5-11 Tuned gas-liquid relative permeability curves of Test $2(3 \mathrm{kPa} / \mathrm{min})$

Figure 5-12 Tuned gas-liquid relative permeability curves of Test 3 (5 kPa/min)

Figure 5-13 Tuned gas-liquid relative permeability curves of Test 4 (12.5 $\mathrm{kPa} / \mathrm{min})$ 60

Figure 5-14 Tuned gas-liquid relative permeability curves of Test $5(50 \mathrm{kPa} / 4$ $\min )$ 61

Figure 5-15 Tuned gas-liquid relative permeability curves of Test $6(100 \mathrm{kPa} / 4$ $\min )$ 61

Figure 5-16 Tuned gas-liquid relative permeability curves of Test 7 (500 kPa/5 $\min )$ 62 
Figure 6-1 Additional run with increased $S_{\text {gcrit }}$ (a) cumulative oil and gas productions (b) cumulative producing GOR (Test 4)

Figure 6-2 Additional run with increased $S_{\text {gcrit }}$ (a) cumulative oil and gas productions (b) cumulative producing GOR (Test 5) 75

Figure 6-3 Additional run with increased $S_{\text {gcrit }}$ (a) cumulative oil and gas productions (b) cumulative producing GOR (Test 6) 76

Figure 6-4 Additional run with increased $S_{\text {gcrit }}$ (a) cumulative oil and gas productions (b) cumulative producing GOR (Test 7)

Figure 6-5 Critical gas saturation and low mobility gas saturation.... 82

Figure 6-6 Simulation results of Test 3 (a) oil and gas production profiles (b) tuned gas-liquid relative permeability curves in production stages 88

Figure 6-7 Additional run of Test 3 by replacing the $\mathrm{k}_{\text {rog_prd }}$ and $\mathrm{k}_{\mathrm{rg} \_p r d}$ curves with the ones of Test 4 (a) cumulative oil and gas productions (b) cumulative producing GOR 91

Figure 6-8 Tuned gas-liquid relative permeability curves for (a) Test 5 (50 kPa/4 min) and (b) Test $6(100 \mathrm{kPa} / 4 \mathrm{~min})$ 94

Figure 6-9 Tuned gas-liquid relative permeability curves in injection stages of Test 7

Figure 6-10 Additional run of Test 5 by replacing the $\mathrm{k}_{\text {rog_inj }}$ and $\mathrm{k}_{\mathrm{rg} \_ \text {inj }}$ curves with the ones of Test 7 (a) cumulative oil and gas productions (b) cumulative producing GOR 97 
Figure 6-11 Additional run of Test 5 by replacing the krog_inj and krg_inj curves with the ones of Test 7: cumulative gas injection................................. 98 


\section{List of Tables}

Table 3-1 Distinguishing experimental set-up and type of data generated ........ 28

Table 3-2 Porosity, permeability, and connate water saturation of sandpack

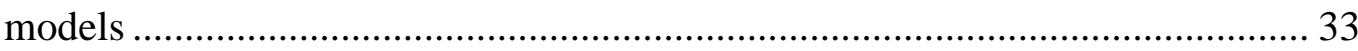

Table 3-3 Dead oil properties at $101.325 \mathrm{kPaa}$............................................ 33

Table 4-1 PVT data of dead heavy oil-propane mixture (provided by SRC)..... 42

Table 6-1 Cumulative producing GOR and recovery factor .......................... 68

Table 6-2 Summary of tuned gas relative permeability curves in production

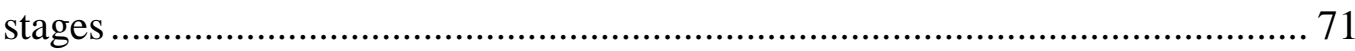

Table 6-3 Dominating differences between this study and Ostos et al. (2004) 


\section{Nomenclature}

\begin{tabular}{|c|c|}
\hline $\mathrm{K}_{\mathrm{rg}}$ & gas relative permeability \\
\hline $\mathrm{K}_{\mathrm{rgcl}}$ & $\mathrm{K}_{\mathrm{rg}}$ at connate liquid saturation \\
\hline $\mathrm{K}_{\mathrm{rog}}$ & gas-oil oil relative permeability \\
\hline $\mathrm{K}_{\mathrm{rogcg}}$ & $\mathrm{K}_{\mathrm{rog}}$ at connate gas saturation \\
\hline $\mathrm{N}_{\mathrm{g}}$ & gas relative permeability curve exponent \\
\hline $\mathrm{N}_{\mathrm{og}}$ & gas-oil oil relative permeability curve exponent \\
\hline $\mathrm{P}_{\mathrm{cgo}}$ & capillary pressure between oil and gas phases \\
\hline $\mathrm{P}_{\text {cgo, } \max }$ & $\mathrm{P}_{\mathrm{cgo}}$ at connate liquid saturation \\
\hline $\mathrm{S}_{\mathrm{g}}$ & gas saturation \\
\hline$S_{\text {gcrit }}$ & critical gas saturation \\
\hline $\mathrm{S}_{\mathrm{gcon}}$ & connate gas saturation \\
\hline $\mathrm{S}_{1}$ & liquid saturation \\
\hline $\mathrm{S}_{\mathrm{l}}^{*}$ & normalized liquid saturation \\
\hline$S_{\text {org }}$ & gas-oil residual oil saturation \\
\hline
\end{tabular}


$\mathrm{S}_{\mathrm{wcon}}$

$\lambda$ connate water saturation

capillary pressure curve constant 


\section{Chapter 1. Introduction}

\subsection{Cyclic Solvent Injection}

It is reported that Saskatchewan occupies approximately $62 \%$ of Canada's total heavy oil resources, which includes 1.7 billion $\mathrm{m}^{3}$ of proved reserves and 3.7 billion $\mathrm{m}^{3}$ of probable reserves (Bowers et al., 1997). Among the Saskatchewan's proven initial heavy oil in place, $97 \%$ resides in reservoirs with a pay zone less than $10 \mathrm{~m}$ and $55 \%$ in reservoirs with a pay zone less than $5 \mathrm{~m}$ (Saskatchewan Energy and Mines, 2000). These heavy oil reserves in thin formations are primarily produced under heavy oil solution gas drive referred to as Cold Heavy Oil Production with Sands (CHOPS). However, it has been suggested that CHOPS only recovers only $5 \sim 10 \%$ of the original oil in place (Chang et al., 2013). As a result, a tremendous amount of untapped heavy oil deposits still remains to be exploited by an effective post-CHOPS recovery method.

The post-CHOPS reservoirs are characterized as thin and unconsolidated formations with a strong wormhole network as well as highly viscous heavy oil. The wormhole networks represent open channels in communication with each other created since the unconsolidated formation has led to sand productions during the CHOPS process. The highly viscous heavy oil corresponds to a high mobility ratio between an injectant (gas or water) and in-situ heavy oil. 
Such post-CHOPS conditions ultimately benefit the solvent-based processes while being unfavorable for the steam-based processes (Dong et al., 2006). The solvent-based processes are desired due to the increased solvent injectivity, resulting in a greater contact area between the solvent and the heavy oil. The steam-based processes, however, are unsuitable due to the considerable heat loss to overburden and underburden. Among the solvent-based processes, the gravity drainage and continuous injection processes are further excluded. The gravity drainage process is deemed inefficient because the thin formations yield low gravity head. The continuous injection process is also ineffective as the high mobility ratio causes a high degree of viscous fingering and therefore an early breakthrough.

Cyclic Solvent Injection (CSI) is a solvent-based process with relatively weak dependency on the gravity drainage. Moreover, it produces the heavy oil solely by a pressure drawdown without any concurrent injection into the reservoir. Consequently, CSI has emerged as an effective post-CHOPS recovery method. A full understanding of the CSI process is immediately required as it will enable exploiting the vast amount of the heavy oil deposits left underground. It is noteworthy that such potential of CSI has led to the field tests on heavy oil reservoirs in Saskatchewan, piloted by Nexen at Plover Lake (Saskatchewan Petroleum Research Incentive, 2006), and Husky at Mervin (Saskatchewan Petroleum Research Incentive, 2011). 


\subsection{Field Design for Heavy Oil Recovery}

CSI is a huff-n-puff process operated cyclically by a single well with the use of solvent as the injectant. When this process is applied in a field scale for heavy oil recovery, the primary design considerations are the areal dispersal of the solvent, volumetric mixing between the oil and the solvent, and retrieval of the solvent. An improvement in these factors advantages the economic viability of the recovery process. Therefore, the design of a CSI cycle and the solvent aims for a quantitative increase in one or more of these factors.

A CSI cycle consists of three sequential stages: injection, soaking, and production, in the order of precedence. In the injection stage, the solvent is forced into a heavy oil reservoir until the porous medium is pressurized to a desired level. The pressure as high as initial reservoir pressure is preferred for a sufficient degree of solvent solubility. Upon the cessation of the injection, the heavy oil-solvent structure enters the soaking stage. This quasi-static period allows the in-situ pressure to stabilize, and the solvent to further dissolve into neighboring oil. In the production stage, a drawdown pressure is imposed at approximately $50 \sim 500 \mathrm{kPaa}$ (Chang et al., 2013). The large pressure difference, which is common in heavy oil recovery, induces the inflow of the solvent-soaked heavy oil. The solvent in the form of free gas flows back simultaneously. The retrieved solvent is recycled in the following cycles. 
In general, light hydrocarbons (e.g. methane, propane, etc.) and carbon dioxide are employed as a pure or mixed solvent. The mixture composition is designed in a manner that the gaseous compound at atmosphere is put slightly below dew point at the end of the injection stages. In this phase equilibria, the injectant is equipped with a tendency to liquefy, and yet the nature of gas is preserved to the utmost. As a result, the solvent attains a high solubility while its inventory in the oil phase is minimized apart from the dissolved portion. The solvent consequently dilutes heavy oil without being excessively liquefied. At the same time, the dissolved quantity is effectively retrieved with the aid of the high gas mobility. When a strong wormhole network is present, the injection may not achieve the initial reservoir pressure. However, CSI can still perform at a lower pressure by designing the solvent to suit the expected condition (Chang et al., 2013).

\subsubsection{Mechanisms}

The mechanisms of CSI involve those of solvent-based processes and heavy oil solution gas drive. The mechanisms of heavy oil solution gas drive take place because the recovery process of CSI is absent of an external driving source in the production stages, which in turn leads to in-situ pressure depletion. Therefore, the solvent dissolved in heavy oil not only functions to add light components in oil phase but also to restore solution gas oil ratio (GOR). Consequently, the CSI process includes the solvent-based as well as the solution gas-based mechanisms, 
which are summarized into: oil viscosity reduction, surface tension reduction, oil swelling, and foamy oil flow.

The oil viscosity is reduced primarily as the molar density of the heavy oil decreases with increasing light solvent components in the oil phase. It then decreases dramatically further through deasphalting process if the solvent concentration increases above a critical point (Luo et al., 2007). The surface tension is reduced since the solvent miscible in oil exists in the gas phase interfacing the oil. A consequence is that the capillary imbibition of the oil is weakened against gravity force, particularly aiding the gravity drainage process. The oil swelling refers to the expansion in the oil volume due to the addition of solvent molecules. The expanded volume mobilizes the residual oil trapped in inaccessible pore spaces. The foamy oil flow is a unique characteristic of heavy oil solution gas drive. The mechanisms associated with this nonequilibrium gasoil flow are explained in the following section.

\subsection{Foamy Oil Flow}

The foamy oil flow appears as the dispersed gas-oil flow in which the gas in the form of microbubbles migrates in line with the oil rather than flowing independently as a continuous phase. Maini et al. (2010) delineated the generation of the foamy oil flow based on the evolution process of the gas bubbles, divided into: bubble nucleation, bubble growth, bubble trappingmobilization, and bubble coalescence/breakup. 
The bubbles nucleation takes place in oil phase when the local pressure declines below the thermodynamic bubblepoint pressure of the oil. The underlying phenomenon is that the oil becomes supersaturated at this pressure level. The bubbles then grow in size following the increase in mass as well as expansion in volume. The mass increases as the gas still dissolved in the adjacent oil phase diffuses into the bubbles. The volume expands in compliance with the pressure decrement. In heavy oil systems, the growing bubbles commence migrating once reaching to a certain size. The bubbles flow takes place within the oil phase in a trapped form. The entrained bubbles keep growing by coalescence and simultaneously breakup into smaller ones. The bubbles eventually develop a continuous gas phase at a local pressure below the pseudobubblepoint pressure of oil. Kraus et al. (1993) introduced the concept of the pseudobubblepoint pressure of oil below which the supersaturated oil releases the free gas.

The foamy oil flow that renders the fluid distribution incompliant with the conventional theories yields the abnormally productive heavy oil wells during the primary cold production. In a reservoir scale, the nonequilibrium between free gas and solution gas results in an unusually low producing GOR and also a significant delay in the reservoir pressure dissipation. The low free gas saturation leads to low gas fractional flow, and moreover, the entrapped gas bubbles innately have very low mobility. Therefore, the foamy oil flow leads to inferior gas phase mobility, preserving the gas and thus reservoir energy in the porous medium. The foamy oil flow consequently improves the heavy oil production in 
a manner not observed in conventional oil wells. In a pore scale, the gas bubbles grow in size in the pore bodies without vacating the spaces initially filled with the heavy oil. The growing gas bubbles by that means displace the heavy oil compounds and advance the heavy oil displacement.

Overall, the foamy oil flow has been found to be a function of oil viscosity, asphaltene content, saturation pressure (or solution GOR), and pressure depletion rate (Maini et al., 2010). Many relevant studies contain more or less conflicting findings. However, a general consensus is that the properties of the foamy oil flow are strongly dependent on the pressure depletion rate. The other factors apart from the pressure depletion rate have shown the effects that are either minor or inconsistent between studies.

\subsection{Gas-Oil Flow in CSI}

In CSI, the injection of solvent that maintains gas state at in-situ conditions spatially divides the porous medium into two zones of differing fluids properties, which are the gas zone - also called solvent chamber, and the heavy oil zone. The solvent chamber mainly contains the gaseous solvent in the form of free gas and under the huff-n-puff operation locates at inner region towards the well. The heavy oil zone represents the bulk oil bordering the outer face of the solvent chamber. The solvent chamber grows nearly as much volume as the oil recovered during a production period. The heavy oil zone shrinks proportionally to the portion that has migrated out from the bulk volume. 
The recovery process of CSI is governed by the gas-oil flow as the solvent chamber is predominated by the free gas-oil flow and the heavy oil zone by the dispersed gas-oil flow (i.e. foamy oil flow). The gas-oil flow appears as the combined flow of free gas and foamy oil which have originated at the solvent chamber and the heavy oil zone, respectively. The free gas and foamy oil travel through the solvent chamber alongside of each other in both injection and production stages. In the injection stages, the free gas injected travels across the solvent chamber along with the foamy oil that has remained as unproduced in the gas zone during a previous production period. In the production stages, while the free gas is discharged from the solvent chamber, the foamy oil that has generated from the solvent-enriched heavy oil travels through the gas zone towards the well. The flow paths are formed across the solvent chamber in accordance with the potential gradients that traverse the inner-located gas zone in both injection and production stages.

The gas-oil flow in CSI results in the characteristics that depend on the properties of the solvent chamber, such as its volume and geometry (i.e. dimensions and location compared to the wellbore), as well as the pressure depletion rate. In general aspects, the solvent chamber serves as the free gas storage in the porous medium and the porous paths that the moving fluids encounter. Therefore, its volume and geometry determine to a large extent the quantity of the free gas and the configuration of the flow paths, respectively. Consequently, the properties of the solvent chamber affect the gas-oil flow by 
involving the effects on the relative quantity of a fluid to another and flow resistance along the flow paths. The pressure depletion rate also influences the gas-oil flow in which the oil phase is mainly the foamy oil. The pressure depletion rate largely determines the nature of the foamy oil flow, as previously discussed.

\subsection{Gas-Oil Flow in CSI versus in Heavy Oil Solution Gas Drive}

The gas-oil flow in heavy oil systems has been widely studied based on heavy oil solution gas drive (Kumar et al., 2002; Ostos et al., 2004; Maini et al., 2010). However, the gas-oil flow in CSI considerably differs from that in heavy oil solution gas drive. The differences mainly arise from the origins of the free gas. In CSI, the free gas originates from the solvent chamber, while in heavy oil solution gas drive, it evolves from solution gas. The origin of the free gas then determines the dependency of the occurrence time and quantity of the free gas on the pseudobubblepoint pressure of oil. As a consequence, the free gas with the distinct characteristics completely differentiates the gas-oil flow in the combined form of free gas and foamy oil.

In CSI, the free gas originates at the solvent chamber of which the gas is supplied from an external source. The combined flow of free gas and foamy oil therefore occurs from the beginning at the in-situ pressure higher than the pseudobubblepoint pressure of the oil. Consequently, the free gas always amounts in addition to the continuous gas phase that develops from the dispersed 
gas bubbles. While the free gas is present, the foamy oil undergoes when the dispersed gas bubbles nucleate, grow, and sustain strong nonequilibrium nature against thermodynamic phase behavior.

In heavy oil solution gas drive, on the other hand, the free gas evolves from solution gas. The combined flow of free gas and foamy oil therefore takes place relatively late at the in-situ pressure lower than the pseudobubblepoint pressure of the oil. As a consequence, the free gas amounts virtually equivalent to the continuous gas phase that develops from the dispersed gas bubbles. While the free gas is present, the foamy oil only undergoes when the dispersed gas bubbles have diminishing nonequilibrium nature in favor of thermodynamic phase behavior.

In summary, the gas-oil flow in CSI considerably differs from that in heavy oil solution gas drive with regard to the combined flow of free gas and foamy oil. The differences appear in the significant aspects of occurrence time, quantity of the free gas, and behavior of the foamy oil. Overall, the gas-oil flow in CSI always results in the combined flow of free gas and foamy oil that is far more susceptible to the quantity of the free gas and the nonequilibrium nature of the dispersed gas bubbles.

\subsection{Problem Statement}

The successful application of CSI greatly depends on the understanding of the gas-oil flow in the combined form of free gas and foamy oil which have 
originated from different sources. The gas-oil flow in CSI governs the recovery process in both injection and production stages and is completely distinguished from that in heavy oil solution gas drive. In particular, the gas-liquid relative permeability curves need to be thoroughly understood under the effect of the growing solvent chamber and the pressure depletion rate. Until now, there has been no study specifically examining the two-phase fractional flow curves in CSI process.

\subsection{Objectives}

This study is aimed at investigating the characteristics of the gas-oil flow in CSI. The particular objective is:

- to investigate the gas-liquid relative permeability curves and understand the effects of the pressure depletion rate on these fractional flow curves; and

- to investigate the gas-liquid relative permeability curves and understand the effects of the solvent chamber on these fractional flow curves.

\subsection{Methodologies}

In this study, numerical simulations were utilized to infer the gas-liquid relative permeability curves. The modified fractional flow model was applied to take into account the foamy oil flow. These methodologies were selected to be more suitable than the others for fulfilling the objectives of this study. The 
methodologies in existence are explained as follows to provide the reasonings for the selections.

When inferring two-phase relative permeability curves for heavy oil systems, a selection is needed between two methodological tools: numerical simulations and analytical two-phase flow equations derived from Darcy's Law. In case CSI is the objective recovery process, the application of numerical simulations is more appropriate and currently a sole approach. The main reason is that the numerical simulations are comprehensive in solvent mass transfer mechanisms, which is intrinsic in CSI process, whereas the analytical flow equations are not. Until now, to the author's knowledge, there has been no attempt to analytically solve the two-phase relative permeability curves in CSI process.

The numerical simulations of CSI process unavoidably necessitate the incorporation of a foamy oil flow model. The existing models are summarized into two broad categories: equilibrium and kinetic (Maini, 2001) based on the time dependency of the resulting phase behavior. These models are built upon the empirical adjustments to the conventional theories. A reliable theoretical solution for the foamy oil flow is not yet available.

The equilibrium models assume the complete and instantaneous equilibrium between phases. The nonequilibrium behavior of the foamy oil flow is incorporated through the adjustments to the fluid or rock-fluid properties. Under this category, the pseudobubblepoint and modified-fractional flow models have 
been widely accepted for reservoir simulation studies. The modified properties in these models are, in the order listed: thermodynamic bubblepoint pressure of oil, and relative permeability to gas, respectively. The oil bubblepoint pressure is decreased to a hypothetical pseudobubblepoint to account for the supersaturation of oil. The gas relative permeabilities are reduced to reflect the low mobility of the discontinuous gas phase.

The kinetic models incorporate the chemical equations to account for the timedependent changes in gas dispersion. The oil phase is redefined with a plural number of non-volatile components including the dispersed gas bubbles. A typical example is the dead oil, dissolved gas, and dispersed gas bubbles. The stoichiometry and reaction-rate constants are then adjusted to control the transformation frequencies between these components.

Among the existing foamy oil flow models, the modified-fractional flow model favors over the others in regard to the evaluation of the gas-liquid relative permeability curves in CSI process. The numerical simulations of CSI process inevitably require the tuning of two-phase relative permeability curves. These curves cannot be obtained from laboratory experiments since the analytical flow equations are currently deficient in describing the solvent mass transfer in CSI process. Therefore, all of the existing foamy oil flow models necessarily involve the fractional flow parameters as variables to be tuned. In the modified-fractional flow model, however, these parameters exist as the only variables, whereas in the 
rest models, the additional variables like the thermodynamic bubblepoint pressure of oil are collectively involved in the tuning process. Eventually, in the models other than the modified-fractional flow model, the additional variables yield a higher dimension of the numerical matrix. The complicated numerical matrix consequently hinders the uniqueness of the tuned parameters including the relative permeability curves. As a result, the modified-fractional flow model yields more reliable gas-liquid relative permeability curves than the other models by containing the fractional flow parameters as the only variables to be tuned.

\subsection{Thesis Outline}

Chapter 2 summarizes the literature reviews on the foamy oil flow and the gasliquid relative permeability curves in heavy oil solution gas drive as well as in CSI. In particular, this chapter focuses on the effect of the pressure depletion rate on the foamy oil flow and the gas-liquid relative permeability curves in heavy oil solution gas drive. The numerical simulation studies of CSI are also introduced with respect to the fractional flow model adopted in the simulations. The methodologies taken in the reviewed studies are briefly presented to show the experimental and/or numerical simulations set-up. The important findings are summarized at the end of each section as well as the chapter.

Chapter 3 describes the experimental design of the CSI tests adopted in this study, including sandpacking, sandpack dimensions, sandpack and fluid properties, experimental devices, and procedure of the CSI tests. Such 
experimental design shows the details of the lab-scale CSI process utilized in this study.

Chapter 4 introduces the design of the numerical simulations performed in this study to infer the gas-liquid relative permeability curves. The design of the numerical simulations involves the modeling of grid system, PVT properties, and uncertain parameters apart from the gas-liquid relative permeability curves. It also includes the methodology applied to tune the gas-liquid relative permeability curves. The uncertain parameters in this study other than the fractional flow model are capillary pressure between oil and gas phases and dispersion coefficients of propane in oil phase.

Chapter 5 presents the history-matching results of the numerical simulations, as well as the tuned gas-liquid relative permeability curves. As a consequence, the results of the modified fractional flow models can be found in this chapter.

Chapter 6 provides the discussions on the history-matching results as well as the tuned gas-liquid relative permeability curves. Firstly, the oil and gas production profiles are examined, which raises the need of explaining the solvent mass transfer mechanisms in CSI process. The tuned gas-liquid relative permeability curves are then analyzed at different pressure depletion rates and to identify the effect of the solvent chamber. Such analysis is performed in company with the sensitivity analysis on the tuned gas-liquid relative permeability curves. The results are further compared to the other studies introduced in the literature 
review. As a consequence, this chapter clearly demonstrates the unique characteristics of the gas-oil flow in CSI along with the explanations on the phenomena responsible for the distinct behavior of the gas-oil flow. The core points of the analysis and results are summarized at the end of each section.

Chapter 7 presents the conclusions and the recommendations for the future works. This chapter therefore summarizes the core findings in Chapter 6 .

Lastly, the appendices include the details of the numerical models in the form as incorporated in the STARS data files. 


\section{Chapter 2. Literature Review}

Chapter 2 summarizes the literature reviews on the foamy oil flow and the gasliquid relative permeability curves in heavy oil solution gas drive as well as in CSI. Specifically, this chapter focuses on the effect of the pressure depletion rate on the foamy oil flow and the gas-liquid relative permeability curves in heavy oil solution gas drive. The numerical simulation studies of CSI are also introduced in regard of the fractional flow model adopted in the simulations. The methodologies in the reviewed studies are presented to demonstrate the experimental and/or numerical simulations set-up. The important findings are summarized at the end of each section as well as the chapter.

\subsection{Foamy Oil Flow}

The foamy oil flow exhibits the nonequilibrium nature as a function of pressure depletion rate. Here, a clarification is needed on the methodologies applied when the relevant studies are performed in a sandpack-scale. In these studies, the primary depletion experiments were performed in sandpack models saturated with live heavy oil. The analysis of the experimental data was then carried out by comparing the recovery factors at different pressure depletion rates. A variance in the oil recovery is deemed to reflect that the foamy oil flow has changed its properties under the effect of the pressure depletion rate. 
Maini et al. (2010) investigated the foamy oil flow at different pressure depletion rates in a sandpack scale. The primary depletion experiments were performed in sandpack models. The sandpack models were $2 \mathrm{~m}$ long. The pressure depletion rates approximately ranged from $7 \mathrm{kPa} / \mathrm{min}$ to $170 \mathrm{kPa} / \mathrm{min}$. Methane, ethane, and carbon dioxide were separately recombined with the dead heavy oil as the solution gases. Irrespective of the solution gas, more oil was recovered at a higher depletion rate.

Zhou et al. (2016) also examined the foamy oil flow under the effect of pressure depletion rate in a sandpack scale. Their study also accompanied the primary depletion experiments in sandpack models. The dimension of the sandpack models was $1 \mathrm{~m}$ in length and $3.8 \mathrm{~cm}$ in diameter. The pressure depletion rates ranged from 0.34 to $6.66 \mathrm{kPa} / \mathrm{min}$. In comparison to the study of Maini et al. (2010), methane and propane were utilized as solution gas in pure or mixed form (i.e. methane, propane, and methane-propane mixture). The results showed that depending on the solution gas a different critical depletion rate exists above or below which the recovery factor decreases. The propane-based and mixturebased experiments presented the lowest and the highest critical rates, respectively. The methane-based experiments yielded the trend of recovery factor reasonably in between the pure substances experiments.

Bora et al. (2000) visualized the effect of the pressure depletion rate in a pore scale with the use of transparent glass micromodel. The pressure depletion rates 
approximately ranged from 380 to $3,100 \mathrm{kPa} / \mathrm{hr}$. The solution gas compositions were not presented. However, it is most likely that methane accounted for the largest fraction of the solution gas as is in typical heavy oil solution gas drive. The behavior of the gas-oil flow in slow depletion tests was analogous to the conventional solution gas theories - the gas bubbles grew without migrating until a continuous gas phase was formed. At fast depletion rates, the dispersed gas-oil flow was observed as previously discussed.

The literature review suggests that the current understanding of the foamy oil flow has been built upon the studies of heavy oil solution gas drive (i.e. primary depletion experiments) but not on any other recovery process. Therefore, the foamy oil flow in CSI needs to be investigated to examine the effects distinct from heavy oil solution gas drive. A proper study of this subject requires the use of propane as the solvent in order to identify its unique characteristics. It has been reported that propane, which is widely applied in CSI process, yields the foamy oil flow that behaves differently.

\subsection{Gas-Liquid Relative Permeability Curves in Heavy Oil Solution Gas Drive}

The conventional studies show that, in light oil systems, two-phase fractional flow depends on saturation, saturation history, wettability, and pore structure, but not on fluid viscosities, densities, and flow rates (Ostos et al., 2004). However, in heavy oil systems, the oil viscosity has also been found to affect the gas 
relative permeability. Talabi et al., (2003) and Javadpour et al., (2004) suggested that, in heavy oil systems, the gas relative permeability decreases with increasing oil viscosity. As a similar implication, Li et al. (2016) presented that the gas relative permeability increased distinctly with temperature - the change in temperature accompanied a variation in oil viscosity. By comparison, the oil relative permeability showed only a slight change.

Ostos et al. (2004) presented the changing trend of the gas-liquid relative permeability curves at different pressure depletion rates in a sandpack-scale. The primary depletion experiments were conducted in sandpack models. The sandpack models were $2 \mathrm{~m}$ long in a horizontal layout. Methane was employed as the solution gas. The pressure depletion rates ranged from 0.2827 to 2.2545 $\mathrm{kPa} / \mathrm{min}$. The gas-liquid relative permeability curves were inferred with the use of numerical simulations. It was found that the gas-liquid relative permeability curves vary at the different pressure depletion rates. The results showed that the critical gas saturation increased up to a maximum attainable value (9\%) and remained at the maximum value even if the depletion rate further increased. In addition, at a higher depletion rate, the end-point of the gas curve decreased, as well as the residual oil saturation. In the gas relative permeability curves, the critical gas saturation appeared between 3 and $9 \%$ while the end point varied from 0.08 to 0.8 . The oil relative permeability curves consisted of the residual oil saturation from 40 to $78 \%$ and the end point fixed at 1.0 . 
Kumar et al. (2002) successfully history-matched a field-scale primary cold production with the application of the modified-fractional flow model. In the history-matching process, they first increased the rock permeability in an attempt to account for the sand production. It was then vitally necessary to reduce the gas fractional flow in order to describe the pressure maintenance mechanism. In the tuned gas fractional flow curve, the critical gas saturation was $2 \%$. The gas relative permeability increased abnormally slowly and eventuated in the very low end point of 0.001 ..

In summary, it has been found that the gas relative permeability curve in heavy oil solution gas drive is a strong function of the pressure depletion rate. The critical gas saturation increases with the pressure depletion rate until a maximum value is attained and plateaus at the maximum value regardless of a further increase in the depletion rate. In general, the gas relative permeability develops a lower curve at a higher pressure depletion rate. The modified-fractional flow model has been applicable since the early investigation of Kumar et al. (2002). Overall, the gas relative permeability in heavy oil systems is anomalously low and eventuates in a very low end point.

\subsection{Gas-Liquid Relative Permeability Curves in CSI}

Ostos et al. (2004) suggested that, in heavy oil systems, the relative permeability curves obtained from external gas drive and solution gas drive can be different. Similarly, Maini (1998) claimed that these fractional flow curves should be 
inferred under the driving conditions that fit the target recovery process. Although these studies did not intend to raise the discussion of CSI process, an implication is that the differing sets of two-phase relative permeability curves one set for injection stages and another for production stages - are necessary to properly simulate a CSI process.

Ivory et al. (2009) conducted the numerical simulation study on their lab-scale CSI process. Their experimental set-up consisted of a stepped cone laboratory sandpack in a vertical alignment. The physical model was $3 \mathrm{~m}$ long. The inside diameters of its bottom and top cylinders were $1 \mathrm{~cm}$ and $9.7 \mathrm{~cm}$, respectively. 28 vol. $\% \mathrm{C}_{3} \mathrm{H}_{8}$ and 72 vol. $\% \mathrm{CO}_{2}$ was employed as the solvent mixture. The primary cold production was first implemented. Six CSI cycles were followed. The pressure depletion rate in the CSI cycles was $125 \mathrm{kPa} / \mathrm{day}$. In the subsequent numerical simulations, a $300 * 1 * 1$ radial grid system was constructed. The experimental results of the cumulative oil and gas productions were historymatched. The kinetic foamy oil model was applied in the production stages. Separate sets of relative permeability curves were employed for the CSI cycles. The tuned curves for the injection stages relatively low end point $(\sim 0.6)$. The curves for the production stages noticeably deviated from the typical figures. The deviations in these curves originated from the very low endpoint of the oil curve $(\sim 0.1)$, very slowly increasing oil relative permeability $\left(\mathrm{k}_{\mathrm{rog}}<0.01\right.$ for $\left.\mathrm{S}_{1}<70 \%\right)$, low end point of the gas curve $(\sim 0.7)$, and very slowly increasing gas relative permeability $\left(\mathrm{k}_{\mathrm{rg}}<0.01\right.$ for $\left.\mathrm{S}_{\mathrm{g}}<45 \%\right)$. 
Chang et al. (2013) implemented the field-scale numerical simulations of CSI process. An actual CHOPS well in Lloydminster area was utilized. The oil, water, and sand production profiles of the well during the primary recovery period were history-matched. The post-CHOPS properties obtained from the history-match established the initial conditions for the CSI process. The fine $\left(36^{*} 36^{* 7}\right)$ and coarse $\left(16^{*} 16^{* 7}\right)$ grid systems were built in Cartesian coordinates. $60 \%$ methane and $40 \%$ propane (the type of fraction was not specified) was used as the solvent mixture. Three CSI cycles were simulated. Each production stage continued for 180 days. During the production periods, the earliest reservoir pressure was approximately 3.5 MPaa. A constant drawdown pressure was imposed at 150 $200 \mathrm{kPaa}$. The kinetic foamy oil model was applied in both injection and production stages. For the CSI cycles, two sets of relative permeability curves were incorporated. The gas phase relative permeabilities during the production periods were designed to be very low. In the corresponding curve, the irreducible water saturation was $37 \%$, the critical gas saturation was $5 \%$, and the residual oil saturation was $15 \%$.

Du et al. (2013) conducted an extensive experimental work on lab-scale CSI processes. Zhang et al. (2014) adopted the experimental data for their numerical simulation study on CSI process. The experiments involved sandpack models in a horizontal layout. The physical models were fabricated in three different sizes. The dimensions were, in diameter*length, $3.81 * 30.48 \mathrm{~cm}, 3.81 * 60.96 \mathrm{~cm}$, and $15.24 * 30.48 \mathrm{~cm}$. A horizontal hole was artificially composed in the interior of 
each physical model. The hole, termed "mimic wormhole," served as a wormhole as it exists in post-CHOPS reservoirs. Pure propane was adopted for the solvent. The pressure depletion rate was $500 \mathrm{kPa} / 5 \mathrm{~min}$. The modifiedfractional flow model was applied. To suit the sandpack dimensions, $24 * 10 * 10$, $48 * 10 * 10$, and $10 * 19 * 13$ corner-point grid systems were constructed. The cumulative oil production profiles of the CSI tests were history-matched. In contrast to the aforestated CSI models, only one set of relative permeability curves were utilized for both injection and production stages. The oil curve was in a typical conventional shape. The gas curve consisted of a low endpoint, 0.15 , and about $10 \%$ critical gas saturation. However, because the gas production profiles were not history-matched, the uniqueness of the gas-oil fractional flow model is relatively weak. Therefore, a stronger justification is required to explain the use of an identical relative permeability curves for both injection and production stages.

The literature review shows that in the numerical simulations of CSI process it is practical to use two sets of two-phase relative permeability curves separately for the injection and production stages. In the injection stages, the gas-liquid relative permeability curves were more or less within the range of conventional figures. In the production stages, however, it has been found that both gas and oil relative permeabilities increase very slowly with growing saturation of the corresponding phase, which then eventuate in low end points. The numerical simulation studies of CSI have been performed at a single pressure depletion rate but not at 
different depletion rates. The gas-liquid relative permeabilities, therefore, have not been investigated under the effect of varying pressure depletion rates. Chang et al. (2013) incorporated the kinetic foamy oil model in both injection and production stages, indicating the need of accounting for the nonequilibrium phase behavior in the injection stages as well as the production stages.

\subsection{Summary}

The literature review on the foamy oil flow and gas-liquid relative permeability curves in heavy oil solution gas drive and in CSI are summarized as follows.

- A proper study of the gas-oil flow in CSI necessitates the use of propane as the solvent which results in unique characteristics of the foamy oil flow not observed in heavy oil solution gas drive.

- In heavy oil solution gas drive, the critical gas saturation increases with pressure depletion rate until a maximum value is attained and plateaus at the maximum value regardless of a further increase in the depletion rate. In general, the gas relative permeability develops a lower curve at a higher pressure depletion rate. Overall, the gas relative permeability in heavy oil systems is anomalously low and eventuates in a very low end point.

- In the numerical simulations of CSI process, it is practical to use two sets of two-phase relative permeability curves separately for the injection and production stages. In the injection stages, it has been reported that the gas- 
liquid relative permeability curves are within the range of conventional figures. In the production stages, however, it has been found that both gas and oil relative permeabilities increase very slowly and eventuate in low end points. Lastly, some numerical simulations of CSI process incorporated the kinetic foamy oil model to account for the nonequilibrium phase behavior in the injection stages. 


\section{Chapter 3. Experiments}

This chapter provides the experimental design of the CSI tests applied in this study, including sandpacking, sandpack dimensions, sandpack and fluid properties, experimental devices, and procedure of the CSI tests. Such experimental design reflects the details of the lab-scale CSI process utilized in this study.

\subsection{Background}

This study was founded on the data obtained from the previous CSI experiments (Du et al., 2013, 2014), as well as the recent experiments by Du, which have not been published yet. Nine CSI tests with the same sandpack dimensions were adopted. Propane was utilized as the solvent. Each of the tests physically simulated a lab-scale CSI process until the end of its productivity life. As previously mentioned, the use of propane as the solvent particularly advantages the research studies, including this study, in need of a strong argument that its findings are unique for CSI. Also, the extended exploratory range strongly justifies that the experimental result is unique for that specific recovery process, which then further justifies the history-matching result.

The nine CSI tests were differentiated in two main design considerations: wormhole location and pressure depletion rate, as tabulated in Table 3-1. 
Table 3-1 Distinguishing experimental set-up and type of data generated

\begin{tabular}{|c|c|c|c|}
\hline $\begin{array}{c}\text { Test } \\
\#\end{array}$ & $\begin{array}{c}\text { Wormhole } \\
\text { Location }\end{array}$ & $\begin{array}{c}\text { Pressure Depletion } \\
\text { Rate }\end{array}$ & $\begin{array}{c}\text { Pressure Depletion Rate } \\
\text { Classification }\end{array}$ \\
\hline 1 & Center & $1 \mathrm{kPa} / \mathrm{min}$ & Lowest \\
\hline 2 & Center & $3 \mathrm{kPa} / \mathrm{min}$ & Very Low \\
\hline 3 & Center & $5 \mathrm{kPa} / \mathrm{min}$ & Low \\
\hline 4 & Center & $12.5 \mathrm{kPa} / \mathrm{min}$ & Middle \\
\hline 5 & Center & $50 \mathrm{kPa} / 4$ min & High \\
\hline 6 & Center & $100 \mathrm{kPa} / 4$ min & Very High \\
\hline 7 & Center & $500 \mathrm{kPa} / 5$ min & Highest \\
\hline 8 & Bottom & $500 \mathrm{kPa} / 5$ min & Highest \\
\hline 9 & Top & $500 \mathrm{kPa} / 5 \mathrm{~min}$ & Highest \\
\hline
\end{tabular}


The pressure depletion rates are classified from lowest to highest in comparison between the numerators in the given fraction forms without being reduced by the denominators.

Test 1 to 7 in common were carried out under the center mimic wormhole at the different pressure depletion rates. These tests were utilized to analyze the trends of the gas-liquid relative permeability curves changing with the pressure depletion rate. In these tests, all other experimental conditions apart from the pressure depletion rate were deemed identical. Therefore, any differences in the experimental results were considered to indicate the effect of the pressure depletion rate. Consequently, the gas-liquid relative permeability curves, which are in strong connection with the pressure depletion rate, were tuned separately for the history-matching of these tests.

In contrast, Test 7 to 9 were conducted under the different wormhole locations at the identical pressure depletion rate. These tests were employed to determine and validate the uncertain parameters incorporated as constants in the numerical simulations of all CSI tests. These uncertain parameters were capillary pressure between oil and gas phases and dispersion coefficients of propane in oil phase. In these tests, except for the wormhole location, all other experimental conditions were regarded as the same. Any differences in the experimental results were therefore considered to be solely due to the changes in the wormhole location. In other words, these tests experimentally involved the identical effects of the 
abovelisted uncertain parameters as well as the gas-liquid relative permeability curves. Consequently, the capillary pressure and dispersion coefficients were determined with validation by history-matching the three different experimental results with the same variable matrix.

\subsection{Experimental Design}

This section illustrates the experimental design of the nine CSI tests. The key information is extracted from the previously provided description (Du et al. 2013, 2014) and supplemented if necessary. The recent experiments, which have not been published yet, were also performed under the same design principles except for the wormhole location and pressure depletion rate. The following illustration, therefore, is not limited to a particular test unless otherwise stated.

\subsubsection{Sandpacking and Sandpack Dimensions}

The CSI experiments were performed individually in a cylindrical sandpack model in a horizontal layout with a mimic wormhole located at center, top, or bottom, also positioned horizontally. Figure 3-1 displays the dimensions of the sandpack models as well as the wormholes set at different locations. 
(a)

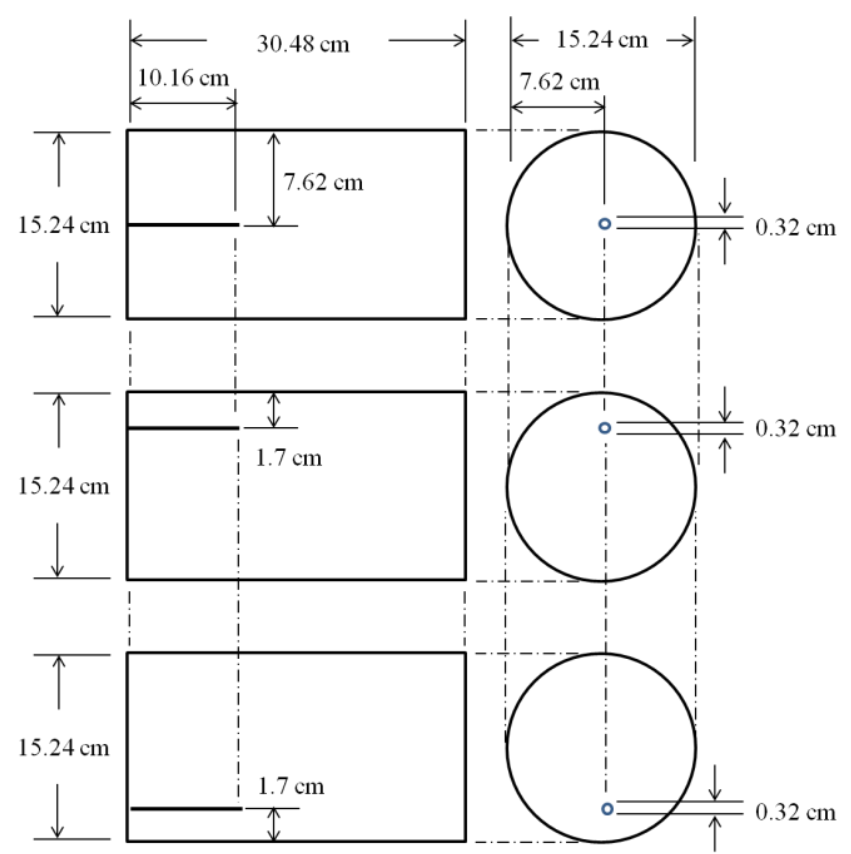

Figure 3-1 Diagram of sandpack models with a mimic wormhole at (a) center; (b) top; and (c) bottom 
The cylindrical sandpack in a horizontal alignment was fabricated by drypacking potters glass beads with an average pore size of $90 \sim 150 \mathrm{~m}$. A spring wrapped by filter gauzes was assembled together. The inner cavity of the spring was devoid of solids during both pre- and post-sandpacking operations. The resulting round hole, defined as a mimic wormhole, started from one of the circular surfaces of the physical model and extended inwards horizontally. The diameter*length of the sandpack and the mimic wormhole were $15.24 * 30.48 \mathrm{~cm}$ and $0.32 * 10.16 \mathrm{~cm}$ (one third of the model length), respectively. The mimic wormhole was located at the center, top, or bottom of the physical model. In the view of the adhering circular base, the center wormhole was set at the origin. The top or bottom wormhole was placed on the vertical centerline such that it was $1.7 \mathrm{~cm}$ away from the neighboring horizontal tangent plane.

\subsubsection{Sandpack and Dead Oil Properties}

After the physical model was sandpacked, it was first completely saturated with water. Then the heavy oil was gently introduced into the porous sandpack until the connate water saturation was achieved. The rock properties and connate water saturations obtained during this period are tabulated in Table 3-2. The properties of the dead oil at different temperatures are presented in Table 3-3. 
Table 3-2 Porosity, permeability, and connate water saturation of sandpack models

\begin{tabular}{|c|c|c|c|}
\hline Test \# & Porosity (\%) & Permeability (D) & Connate Water Saturation (\%) \\
\hline 1 & 34.15 & 5.38 & 2.38 \\
\hline 2 & 35.05 & 5.68 & 8.51 \\
\hline 3 & 33.42 & 5.29 & 4.05 \\
\hline 4 & 34.15 & 5.59 & 3.17 \\
\hline 5 & 33.51 & 5.27 & 7.61 \\
\hline 6 & 33.51 & 5.29 & 4.1 \\
\hline 7 & 33.24 & 5.27 & 5.98 \\
\hline 8 & 33.33 & 5.27 & 5.69 \\
\hline 9 & 33.09 & 5.62 & 6.25 \\
\hline
\end{tabular}

Table 3-3 Dead oil properties at $101.325 \mathrm{kPaa}$

\begin{tabular}{|c|c|c|}
\hline Temperature $\left({ }^{\circ} \mathrm{C}\right)$ & Density $\left(\mathrm{kg} / \mathrm{m}^{3}\right)$ & Viscosity $(\mathrm{cp})$ \\
\hline 15 & 967.9 & 4,330 \\
\hline 25 & 961.8 & 1,830 \\
\hline 75 & 929.5 & 72.3 \\
\hline
\end{tabular}


The physical model, once being sandpacked, was put under vacuum and filled with fresh water. The porosity was derived from quantifying the water that fully saturated the pore volume. Continually, the injection proceeded at varying steady-state rates. The pressure gradient and flow rate measured at a steady-state was substituted into Darcy's Law. The flow equation was then solved for absolute permeability. The computed permeability was compared and verified with the values obtained at the other flow rates. Provided that the acquired rock properties were within the tolerable deviations, the pre-evaluated dead heavy oil was gently introduced into the water-filled pores. The oil imbibition lasted until the connate water saturation was achieved. The porosity and permeability ranged between $33.24 \sim 35.05 \%$, and $5.17 \sim 5.68 \mathrm{D}$, respectively. The high values successfully imitated the characteristics of unconsolidated sediments.

\subsubsection{Experimental Devices}

As the initial conditions of a post-CHOPS system were established, the experimental de-vices for CSI cycles were assembled. A connection port was coupled with the outer tip of the mimic wormhole. A pressure transducer was installed at the port to transmit the inlet or outlet pressure. Another one was located at the center of the opposite surface from the port for the model pressure. The transducers were wired to a computerized system. A programmable back pressure regulator (BPR) was set at a flow line downstream of the port. A conical flask was placed at the end of the flow line. A wet gas flow meter was joined at 
the top of the liquid storage apparatus. All experimental equipments were leakfree. The port linked to the mimic wormhole is termed as the inlet or outlet port, hereafter. The pressure at the adjoining upstream of the BPR is referred to as the drawdown pressure. A note should be made that for Test 7 and 8 the gas flow meter was not available, and for Test 7,8, and 9 the pressure transducer for the model pressure.

\subsubsection{CSI Tests}

Once the experimental devices were set-up, the CSI test was performed in the sandpack model filled with the dead heavy oil and connate water with the use of propane as the solvent. From the start to the end of the test, the temperature was reasonably unchanging at $22{ }^{\circ} \mathrm{C}$ (ambient temperature). The electronic instruments automatically gauged and recorded the pressure specifics into a local computer as frequent as 15 seconds.

In an injection stage, pure propane was introduced into the physical model through the inlet port. The inlet pressure was maintained at $800 \mathrm{kPaa}$. The porous medium was pressurized to the topmost level (i.e. $800 \mathrm{kPa}$ ). The injection lasted for 45 minutes. The physical model was sealed off subsequently. The insitu pressure stabilized at a few kilopascals lower. The soaking stage continued for 10 minutes. The injection pressure was intended to be slightly below the vapor pressure of propane. The time periods for the injection and soaking states were selected to assure the complete pressurization and pressure stabilization. 
A production stage was followed. It was initiated by unlocking the physical model from the outlet port. The physical model was depleted in a stepwise manner. A depletion step consisted of the immediate decrease of the drawdown pressure at the outset, and the subsequent span over which the pressure was fixed. The given fraction expression of the pressure depletion rate quantifies these components. For example, the numerator specifies the degree of the pressure drop, and the denominator the step duration. These values were preprogrammed into the BPR. The drawdown pressure was declined in agreement with the pressure depletion rate. In the event that the oil flux continued, the pressure was reduced as far as the ambient level. The further recovery proceeded at the same conditions. The cycle was ended at any depletion step when the oil ceased to flow. In contrast to the injection and soaking phases, the period of the production stage was unconstrained. It was rather determined by the productivity of the cycle.

The highest (i.e. $500 \mathrm{kPa} / 5 \mathrm{~min}$ ) depletion process was exceptionally operated. The drawdown was maintained at the ambient pressure. The BPR was not applied. This strategy was necessary to prompt the release of a large portion of the initial pressure (i.e. $\sim 800 \mathrm{kPaa}$ ) at the outlet port. The rapid and large pressure drop resulted in the short duration of the oil flow. To ensure the enough exhaustion of the porous medium, the production period was fixed for 5 minutes. The time also allowed the experimenter to perform unhurried hand operations when the cycle transferred to another. 
The oil collected at the bottom of the conical flask was gauged in mass and converted to volume. The exaggerated portion due to the foamy bubbles was excluded by this means. The gas production was measured in volume and read off from the flow meter. The production data were logged once at the end of the cycle. The ensuing cycle was carried out in the identical manner as above. The test was terminated if the oil recovery was trivial for two consecutive cycles. 


\section{Chapter 4. Numerical Simulations}

This chapter introduces the design of the numerical simulations conducted in this study to evaluate the gas-liquid relative permeability curves. The design of the numerical simulations involves the modeling of grid system, PVT properties, and uncertain parameters apart from the gas-liquid relative permeability curves. This chapter also introduces the methodology applied to tune the gas-liquid relative permeability curves. The uncertain parameters in this study other than the fractional flow model are capillary pressure between oil and gas phases and dispersion coefficients of propane in oil phase.

In this study, the commercial numerical simulator, STARS (CMG, 2013), was employed to history-match the experimental results of the CSI tests (i.e. cumulative oil and gas production profiles, and model pressure). The mimic wormhole was defined as a well for both injector and producer. The experimental data of the inlet and outlet pressures at the port are used as the well constraints for both injection and production stags. The bottomhole pressure of the well in the production stages were designed in accordance with the pressure depletion rate controlled by the BPR.

Test 7, 8, and 9 were history-matched preliminarily to determine and validate the capillary pressure and dispersion coefficients as well as to infer the gas-liquid relative permeability curves for these tests. The rest CSI tests (Test 1 to 6 ) were 
then history-matched by applying the validated uncertain parameters and tuning the gas-liquid relative permeability curves.

\subsection{Grid Modeling}

The grid modeling was performed on the basis of the orthogonal corner-point grid system to properly describe the cylindrical sandpack and mimic wormhole in horizontal positions and resulting fluids flow directions. The mimic wormhole was defined as injector and producer. Another well named observer was added for the tests accompanying the model pressure measurements. Figure 4-1 displays the grid system with the mimic wormhole at center as an example. Appendix I presents the coordinates of the grid blocks as incorporated in the STARS data files. 


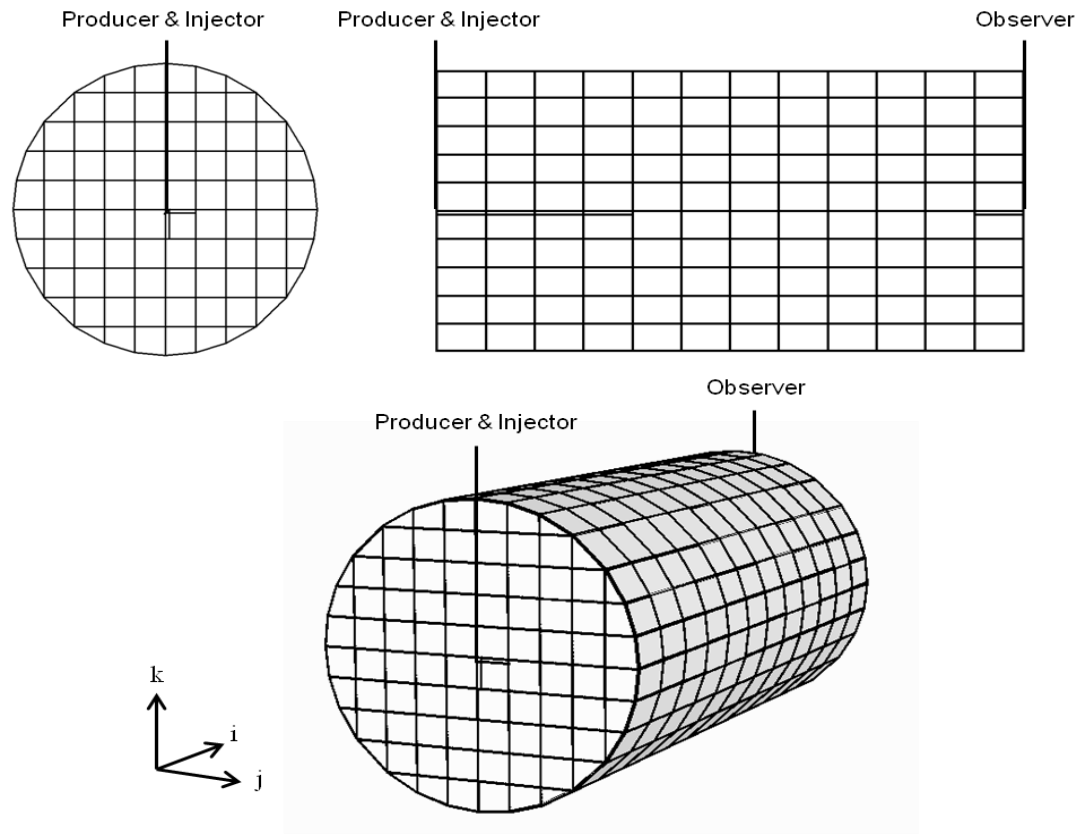

Figure 4-1 Grid system with the well (mimic wormhole) located at center 
The number of the grids was $12 * 10 * 10(2.54 * 1.524 * 1.524 \mathrm{~cm})$ in $\mathrm{i} * \mathrm{j} * \mathrm{k}$ directions. The grid blocks outside the model boundaries were given the null properties. The grid blocks accommodating the injector (or producer) were refined to conform to the location of the mimic wormhole. The dimension of the refined grid blocks containing the well was $2.54 * 0.19 * 0.19 \mathrm{~cm}$ in the Cartesian directions. The diameter of the well was $0.01 \mathrm{~cm}$. With such grid size and well diameter, the location of the mimic wormhole was respected as much as possible, while a sufficiently large Well Index was incorporated. The grid block contacting the observer was refined in the same way. The observer was a shut-in well included to gather the simulated model pressure values. The total number of the grid blocks in effect was 1,071 and 1,068 with and without the model pressure, respectively.

\subsection{PVT Modeling}

The PVT modeling was conducted by matching the dead heavy oil viscosity at different temperatures (Table 3-3) and the dead heavy oil-propane mixture viscosity at varying pressure and temperature (Table 4-1). The PVT properties were obtained from the bulk-volume laboratory experiments at equilibrium conditions. These experiments were implemented by Saskatchewan Research Council (SRC). The matching results are shown in Figure 4-2 and 4-3. Appendix II includes the tables of the K-factors and the oil viscosity as incorporated in the STARS data files. 
Table 4-1 PVT data of dead heavy oil-propane mixture (provided by SRC)

\begin{tabular}{|c|c|c|c|}
\hline $\begin{array}{c}\text { Temperature } \\
\left({ }^{\circ} \mathrm{C}\right)\end{array}$ & $\begin{array}{c}\text { Pressure } \\
(\mathrm{kPaa})\end{array}$ & $\begin{array}{c}\text { Propane Mole } \\
\text { Fraction }\end{array}$ & $\begin{array}{c}\text { Viscosity } \\
(\mathrm{cp})\end{array}$ \\
\hline 15.4 & 300 & 0.38 & 435 \\
\hline 15.4 & 400 & 0.50 & 129 \\
\hline 15.4 & 500 & 0.59 & 49.4 \\
\hline 15.4 & 625 & 0.761 & 7.24 \\
\hline 75.0 & 500 & 0.212 & 40.3 \\
\hline 75.0 & 1,000 & 0.392 & 18.7 \\
\hline 75.0 & 1,500 & 0.545 & 8.66 \\
\hline 75.0 & 2,000 & 0.681 & 3.75 \\
\hline
\end{tabular}

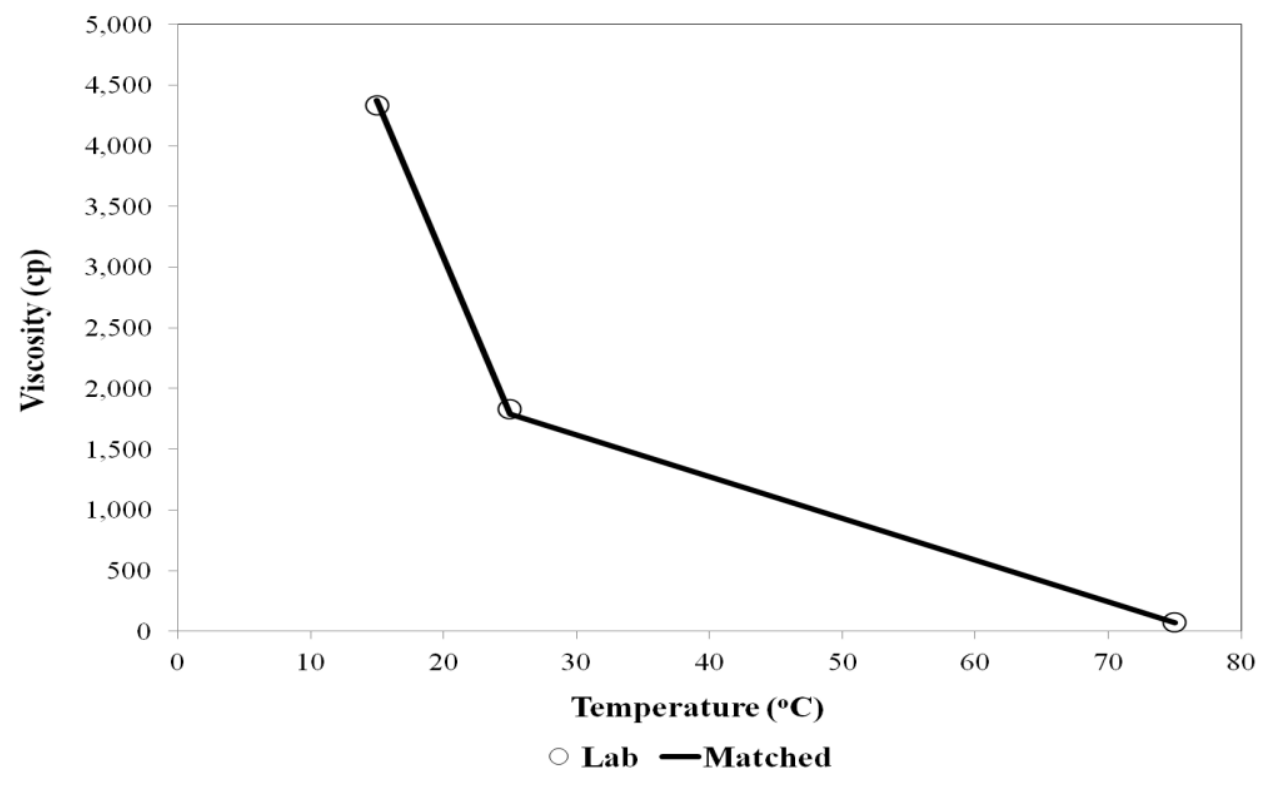

Figure 4-2 Dead oil viscosity matching at 1 atm 


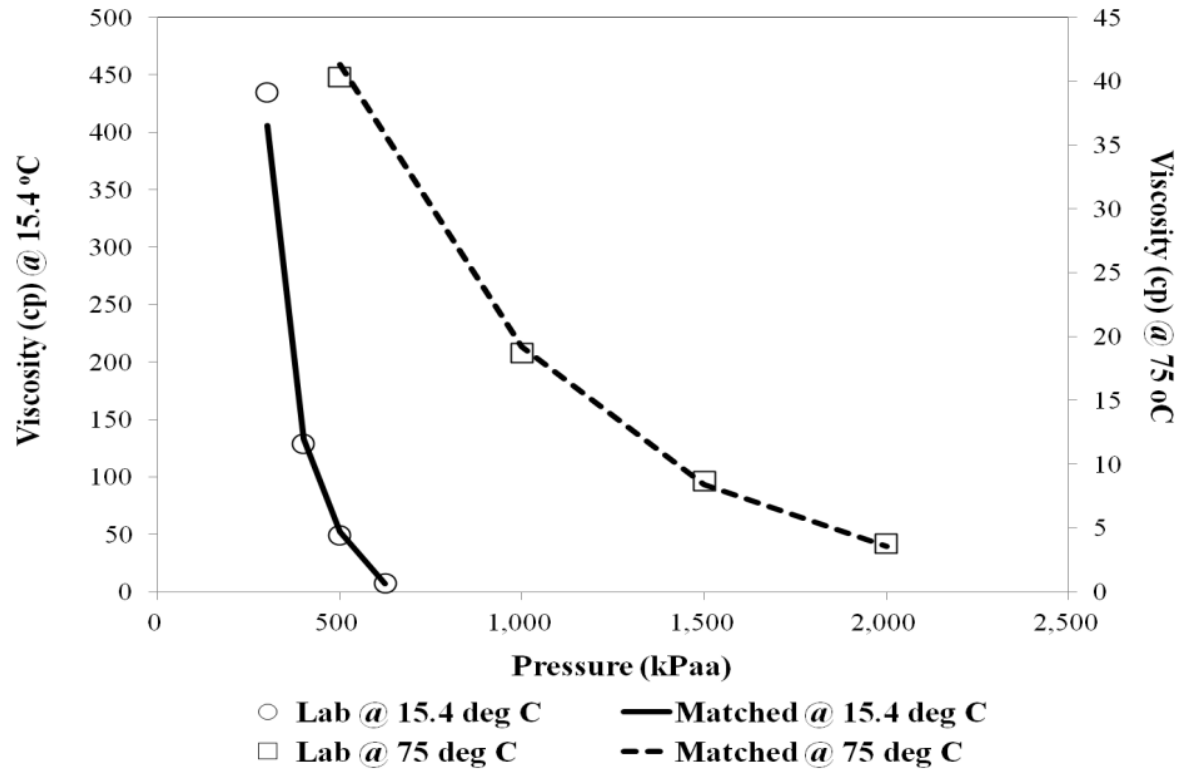

Figure 4-3 Dead oil \& propane mixture viscosity matching at $15.4{ }^{\circ} \mathrm{C}$ and $75{ }^{\circ} \mathrm{C}$ 


\subsection{Capillary Pressure and Dispersion Coefficients}

The capillary pressure curve validated by the preliminary history-matching of Test 1, 8, and 9 is shown in Figure 4-4. The dispersion coefficients of propane in oil phase, validated by the same means, were $2 * 10^{-6} \mathrm{~cm}^{2} / \mathrm{min}$ in all $\mathrm{i}, \mathrm{j}$, and $\mathrm{k}$ directions. The differences in connate water saturation resulted in slightly different capillary pressure curves between the tests. The curve shown in the

figure belongs to Test 5 . It should be noted that the formation compressibility was $4 * 10^{-5} 1 / \mathrm{kPa}$ with the porosity reference pressure at $101 \mathrm{kPa}$. 


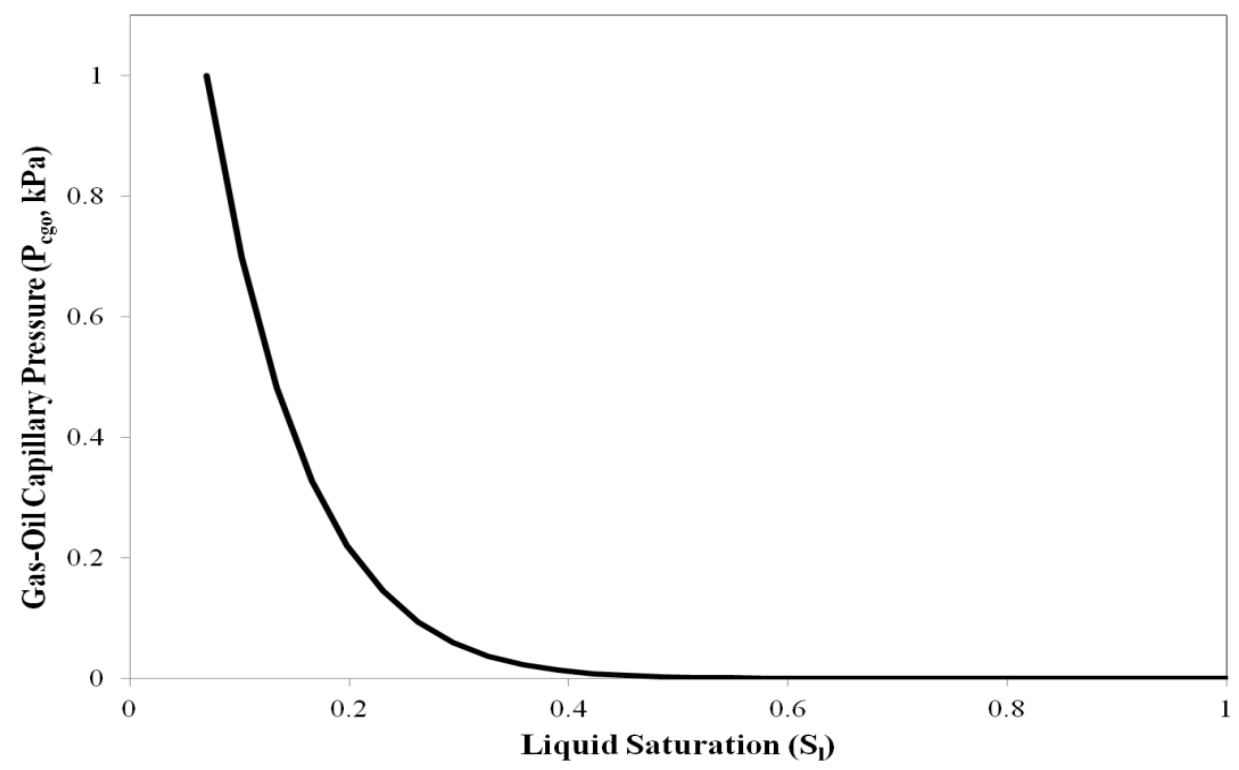

Figure 4-4 Capillary pressure curve between gas and oil phases 
The low range of capillary pressure $(0 \sim 1 \mathrm{kPa})$ was adopted from Ivory et al. (2009). The capillary pressure curves were computed with $\mathrm{Li}$ and Horne's empirical model (2001) with the use of normalized liquid saturation, $S_{l}^{*}$ (Equation 1 and 2).

$$
\begin{gathered}
P_{c g o}=P_{c g o, \max }\left(1-S_{l}^{*}\right)^{-\frac{1}{\lambda}} \\
S_{l}^{*}=\frac{S_{l}-S_{\text {oirg }}-S_{\text {wcon }}}{1-S_{0 i r g}-S_{\text {wcon }}-S_{\text {gcon }}}
\end{gathered}
$$

where $\mathrm{P}_{\text {cgo }}$ : capillary pressure between oil and gas phases; $\mathrm{P}_{\text {cgo, } \max }: \mathrm{P}_{\text {cgo }}$ at connate liquid saturation (sum of gas-oil irreducible oil saturation and connate water saturation); $\lambda$ : capillary pressure curve constant; $S_{1}^{*}:$ normalized liquid saturation; $\mathrm{S}_{1}$ : liquid saturation; $\mathrm{S}_{\mathrm{oirg}}$ : gas-oil irreducible oil saturation; $\mathrm{S}_{\mathrm{wcon}}$ : connate water saturation; and $\mathrm{S}_{\mathrm{gcon}}$ : connate gas saturation

For all tests, the $\mathrm{P}_{\text {cgo, max }}$ was $1 \mathrm{kPa}$. The capillary pressure constant $(\lambda)$ was 0.0979. The connate gas saturation and gas-oil irreducible oil saturation were 0 and 0.01 , respectively. The connate water saturation varied from test to test (Table 3-2).

\subsection{Tuning of Gas-Liquid Relative Permeability Curves}

In this study, two sets of two-phase relative permeability curves were incorporated separately for injection and production stages. To model the gas- 
liquid relative permeability curves, Corey's correlations (Equation 3 and 4) were utilized. These correlations were successful in history-matching Test $1,2,7,8$ and 9. For the rest tests (Test 3, 4, 5 and 6), however, the relative permeability curves in part had to be adjusted manually. The sole application of the correlations could not yield successful history-matching results.

$$
\begin{gathered}
K_{r g}=K_{\text {rgcl }}\left(\frac{S_{g}-S_{\text {gcrit }}}{1-S_{\text {gcrit }}-S_{\text {oirg }}-S_{\text {wcon }}}\right)^{N_{g}} \\
K_{\text {rog }}=K_{\text {rogcg }}\left(\frac{S_{l}-S_{\text {org }}-S_{\text {wcon }}}{1-S_{\text {gcon }}-S_{\text {org }}-S_{w c o n}}\right)^{N_{o g}}
\end{gathered}
$$

where $\mathrm{K}_{\mathrm{rg}}$ : gas relative permeability; $\mathrm{K}_{\mathrm{rgcl}}: \mathrm{K}_{\mathrm{rg}}$ at connate liquid saturation; $\mathrm{S}_{\mathrm{g}}$ : gas saturation; $\mathrm{S}_{\text {gcrit }}$ : critical gas saturation; $\mathrm{N}_{\mathrm{g}}$ : gas relative permeability curve exponent; $\mathrm{K}_{\text {rog: }}$ gas-oil oil relative permeability; $\mathrm{K}_{\mathrm{rogcg}}$ : $\mathrm{K}_{\mathrm{rog}}$ at connate gas saturation; $\mathrm{S}_{\mathrm{org}}$ : gas-oil residual oil saturation; and $\mathrm{N}_{\mathrm{og}}$ : gas-oil oil relative permeability curve exponent

In the application of the Corey's correlations, the gas-relative permeability curves in injection and production stages were tuned in different ways. In the injection stages, the end points, $\mathrm{K}_{\mathrm{rgcl}}$ and $\mathrm{K}_{\mathrm{rogcg}}$, were fixed at 1 . The exponents, $\mathrm{N}_{\mathrm{g}}$ and $\mathrm{N}_{\mathrm{og}}$, were adjusted prior to the critical gas saturation and residual oil saturation. The critical gas saturation and residual oil saturation were increased only if necessary. In the production stages, all fractional flow parameters were 
subject to adjustments. The exponents $\left(\mathrm{N}_{\mathrm{g}}\right.$ and $\left.\mathrm{N}_{\mathrm{og}}\right)$ and end-points $\left(\mathrm{K}_{\mathrm{rgcl}}\right.$ and $\mathrm{K}_{\text {rogcg }}$ ) were adjusted first. The critical gas saturation and residual oil saturation were increased only when imperative. 


\section{Chapter 5. Results}

This chapter presents the history-matching results of the numerical simulations, as well as the tuned gas-liquid relative permeability curves. Accordingly, the results of the modified fractional flow models can be found in this chapter.

\subsection{History Matching Results}

The experimental and history-matching results of the CSI tests are shown in Figure 5-1 to 5-9 in the order of the test number. For the production profiles, the abscissas indicate the times that the solvent cycles were ended. The primary and secondary ordinates show the cumulative oil and gas productions of the cycles, respectively. The model pressure is presented for the beginning five cycles only because a massive number of the data points make the plots undistinguishable. As previously mentioned, due to the deficiency in the measuring devices, the gas production measurements were not gathered in Test 7 and 8 as well as the model pressure data in Test 7, 8, and 9. The unreasonable values of the model pressure were excluded from the plots. 


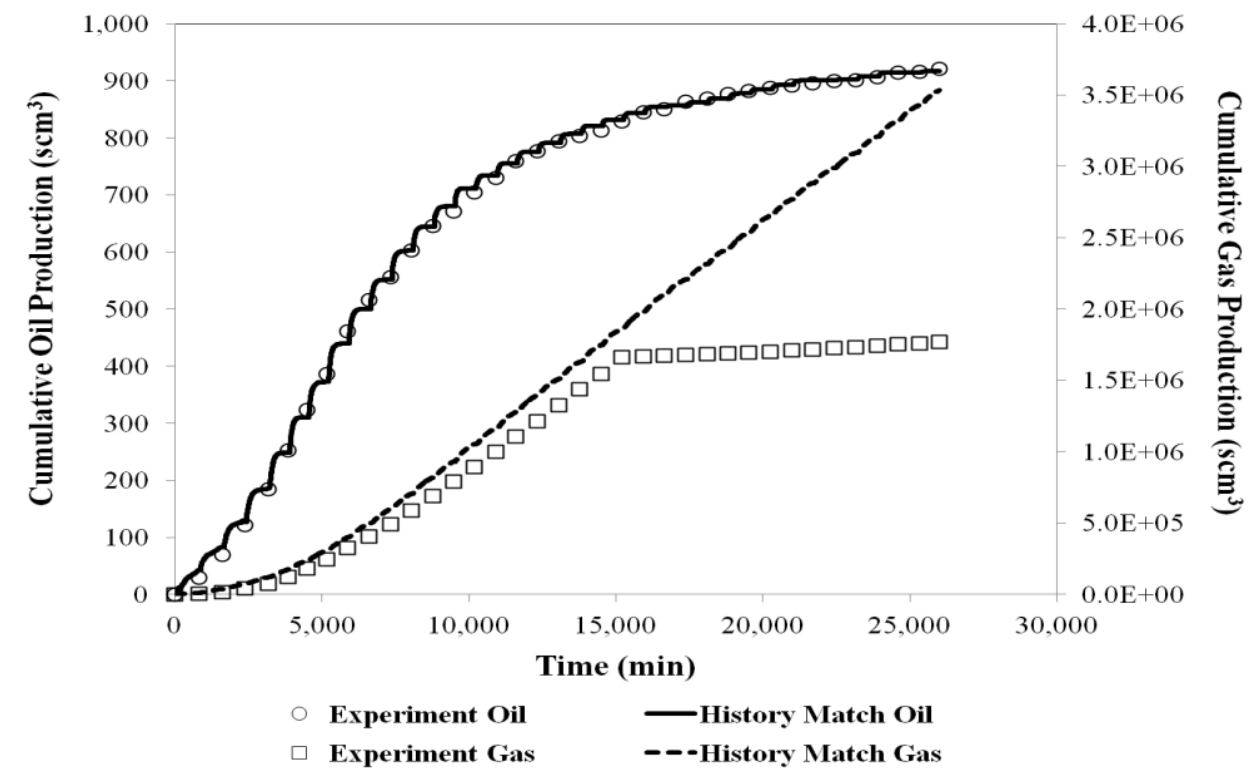

(a)

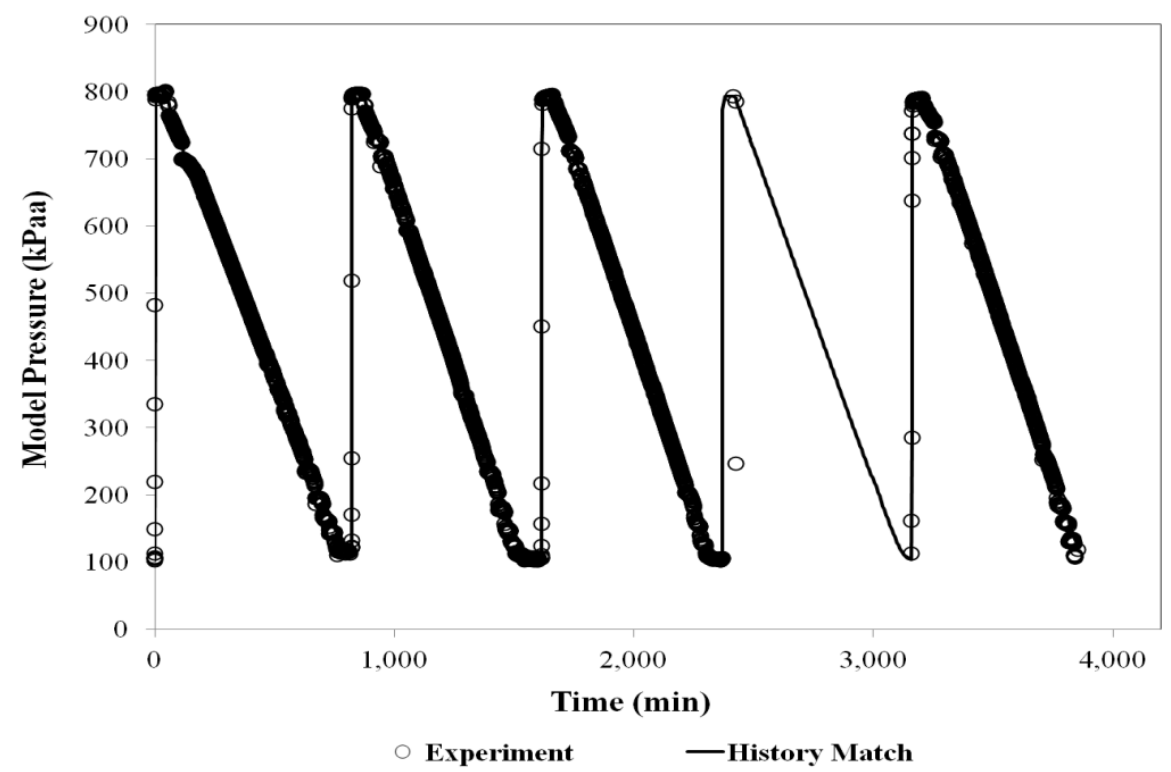

(b)

Figure 5-1 History matching results (a) production profiles (b) model pressure of Test $1(1 \mathrm{kPa} / \mathrm{min}$, center wormhole $)$ 


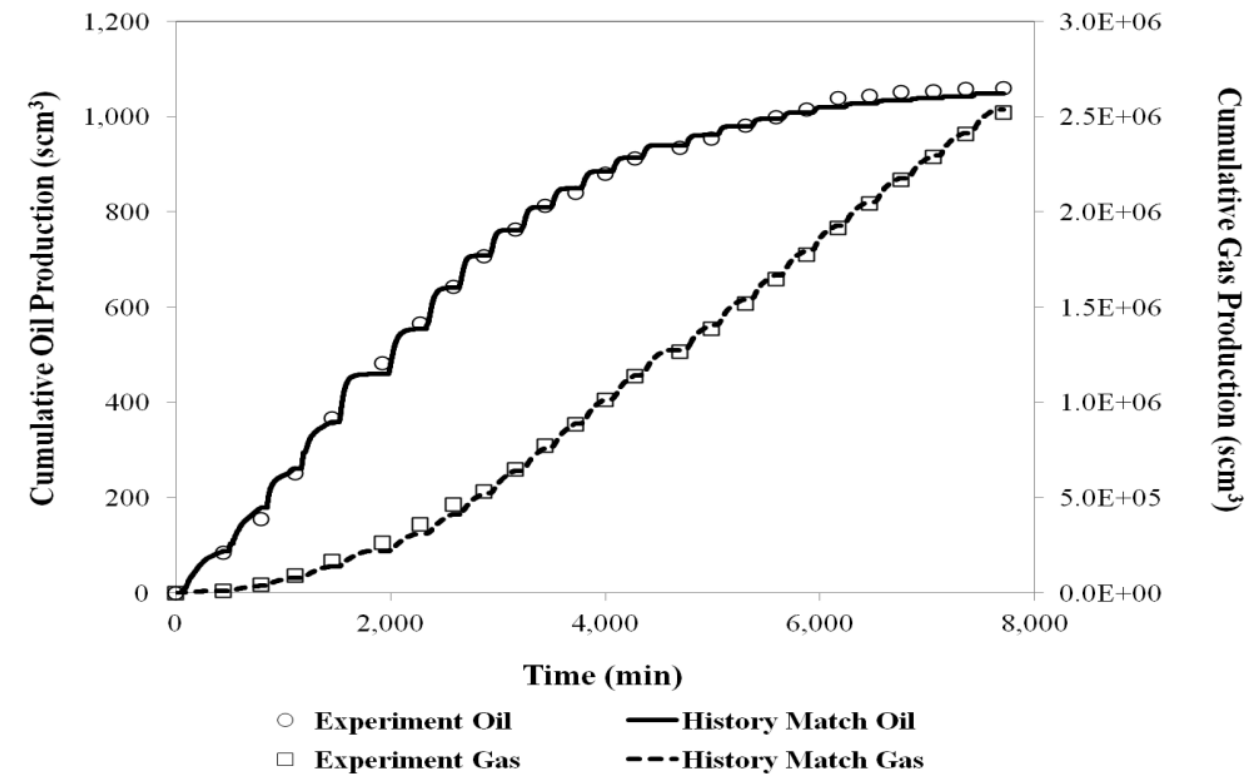

(a)

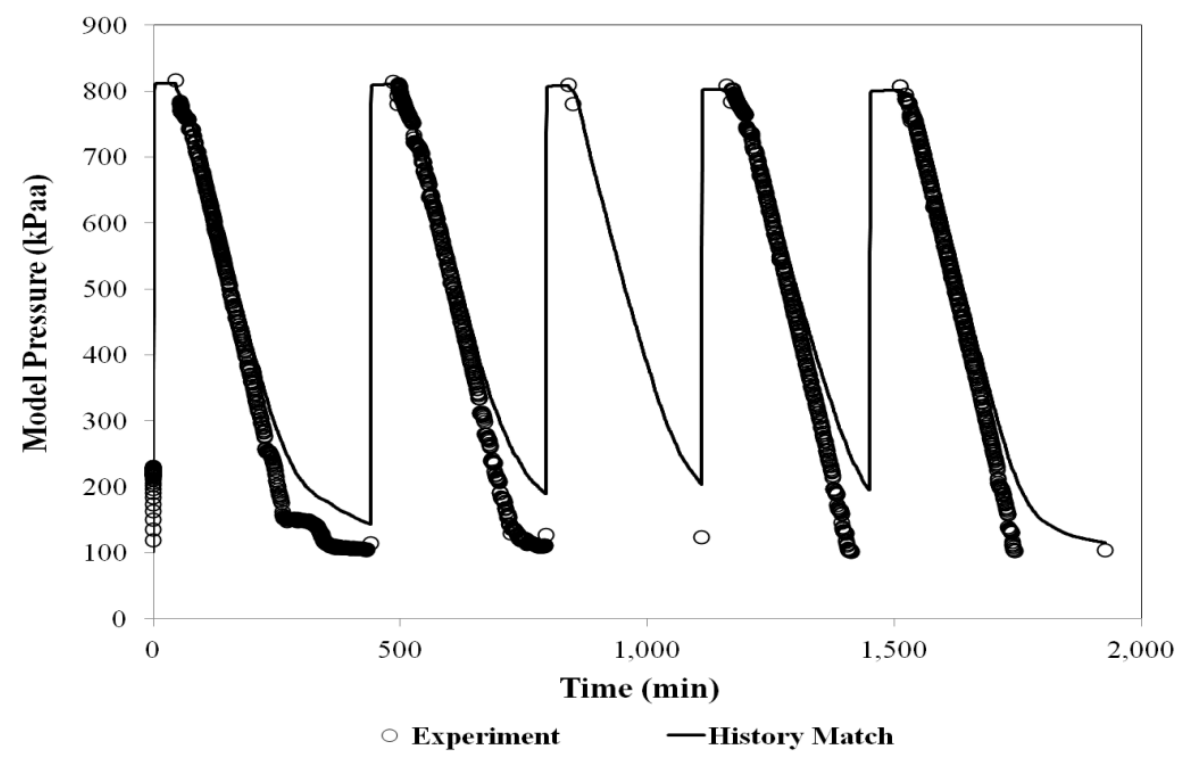

(b)

Figure 5-2 History matching results (a) production profiles (b) model pressure of Test $2(3 \mathrm{kPa} / \mathrm{min}$, center wormhole $)$ 


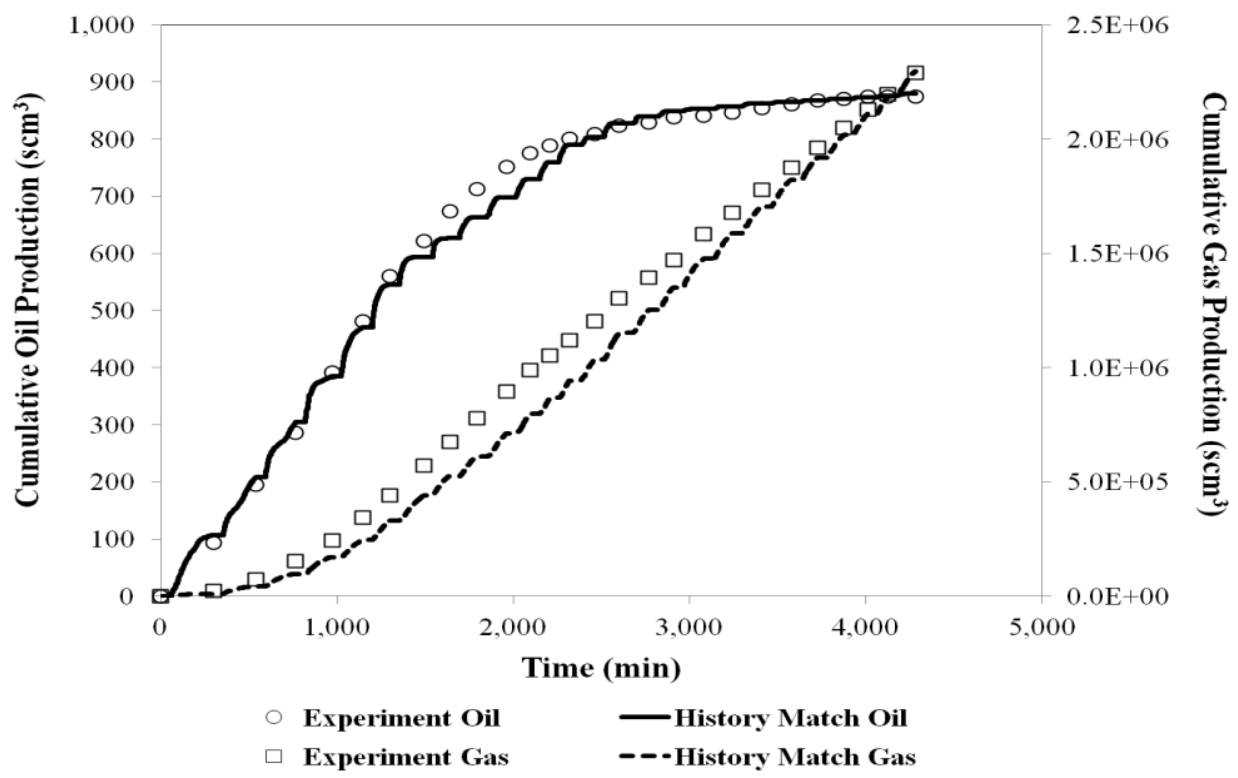

(a)

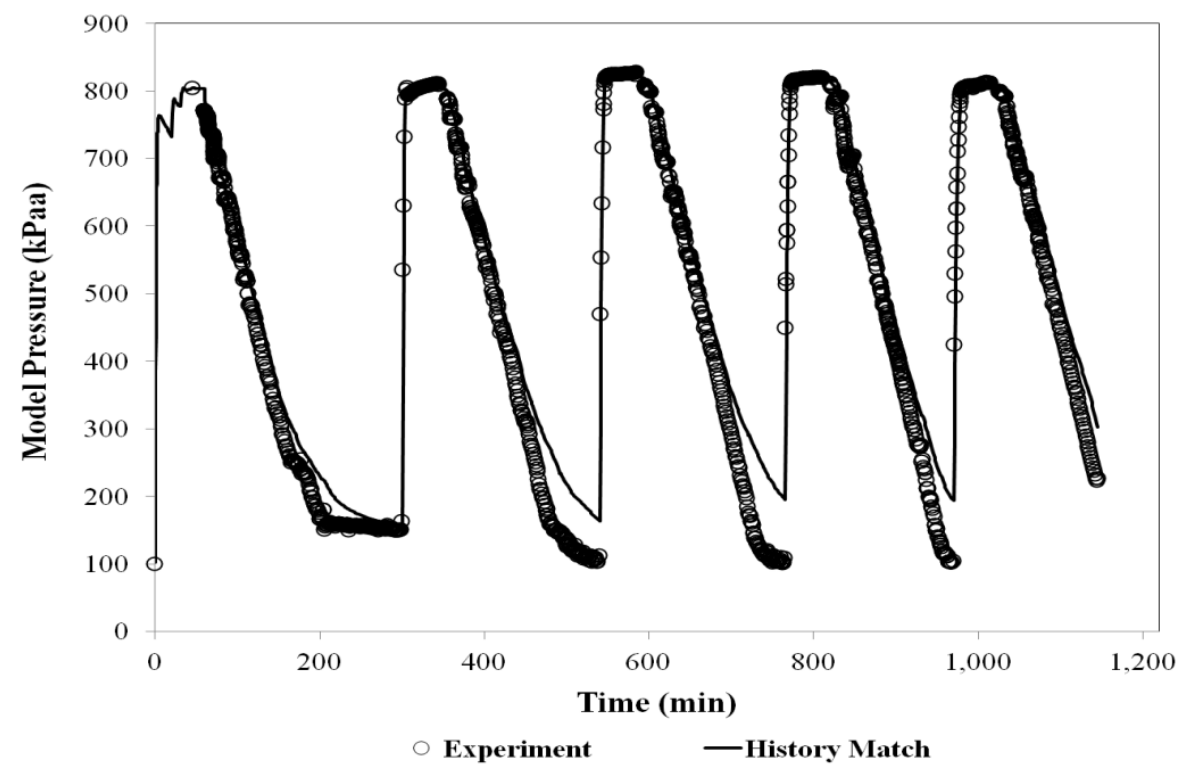

(b)

Figure 5-3 History matching results (a) production profiles (b) model pressure of Test $3(5 \mathrm{kPa} / \mathrm{min}$, center wormhole) 


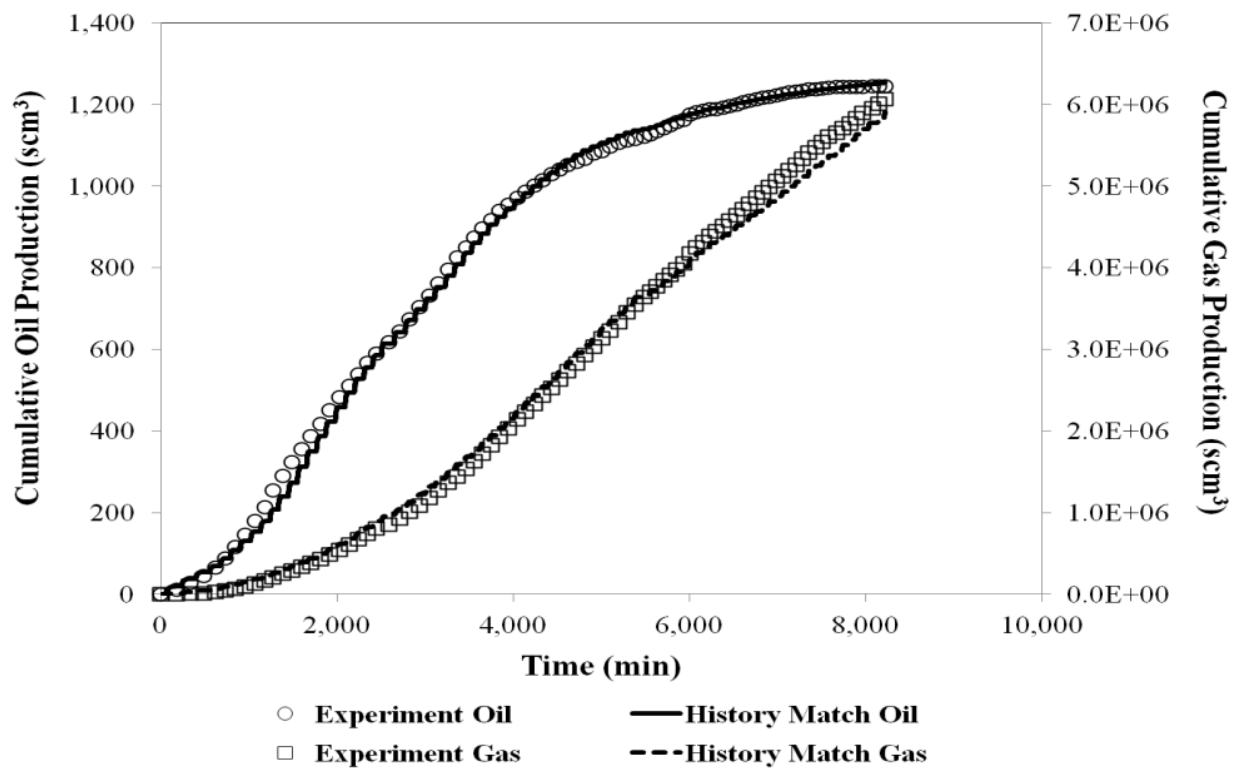

(a)

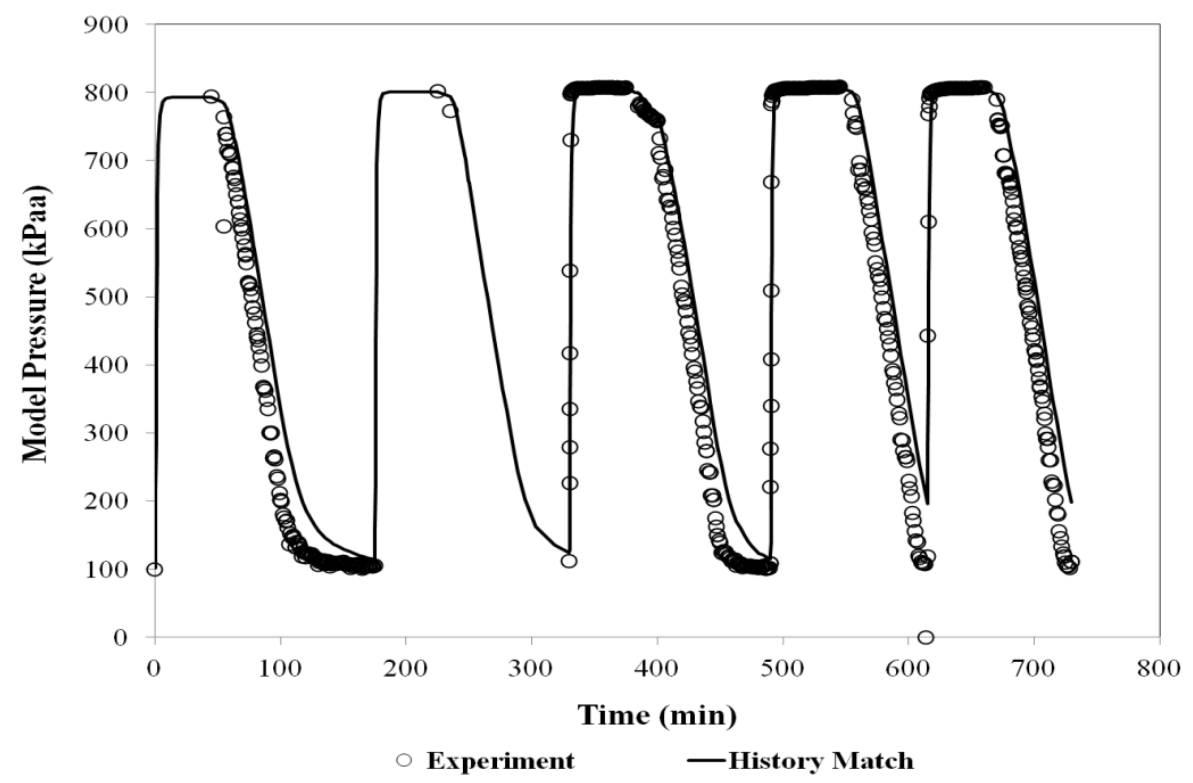

(b)

Figure 5-4 History matching results (a) production profiles (b) model pressure of Test $4(12.5 \mathrm{kPa} / \mathrm{min}$, center wormhole $)$ 


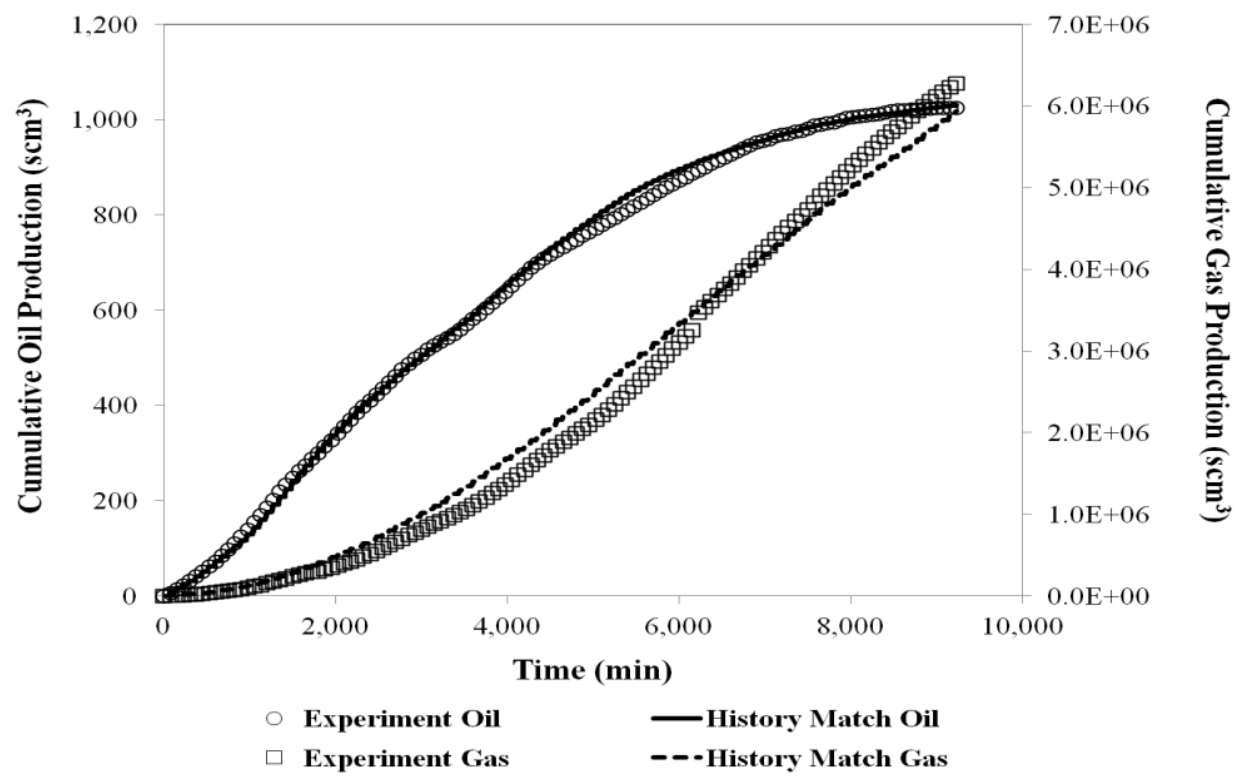

(a)

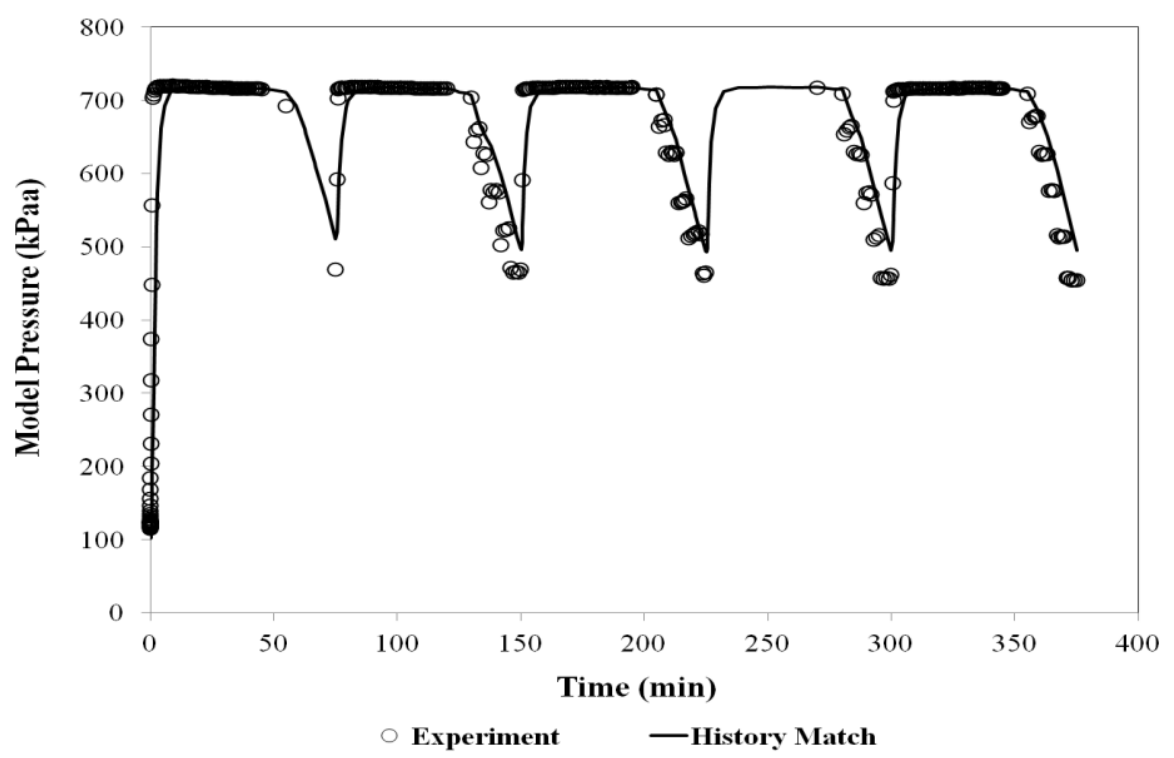

(b)

Figure 5-5 History matching results (a) production profiles (b) model pressure of Test $5(50 \mathrm{kPa} / \mathrm{min}$, center wormhole $)$ 


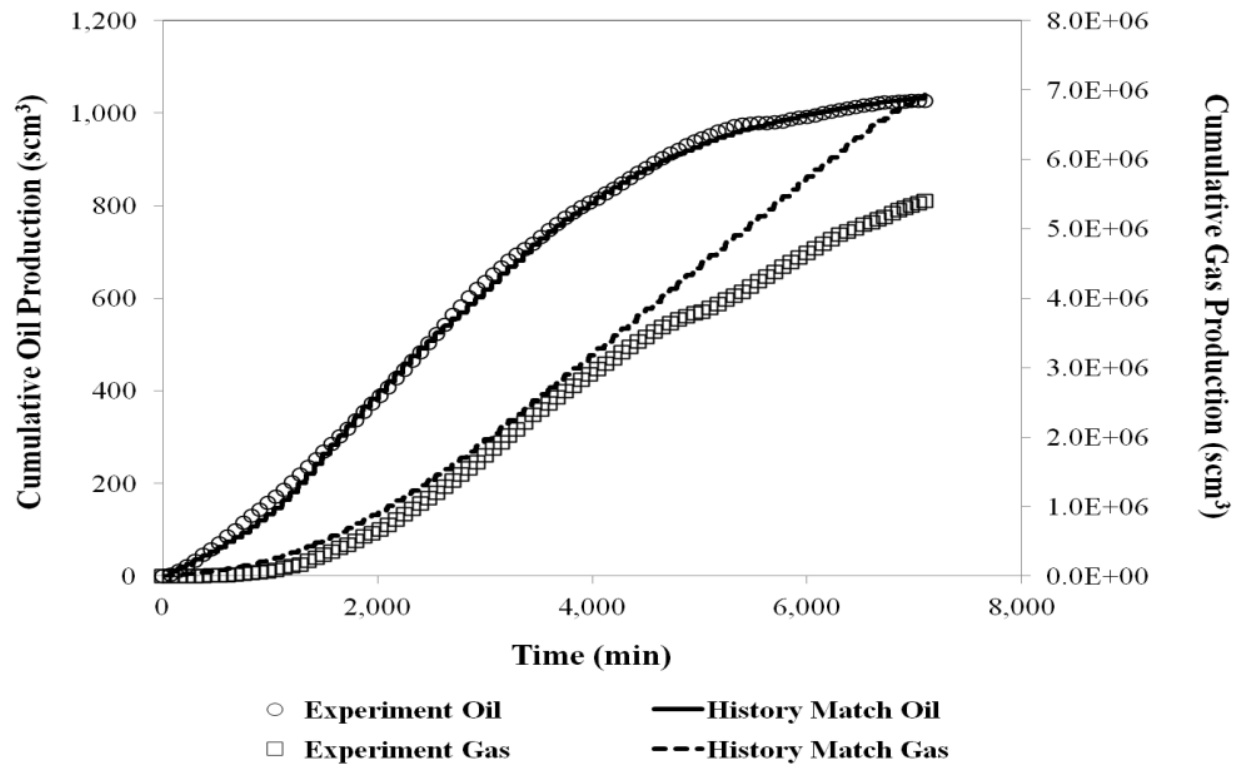

(a)

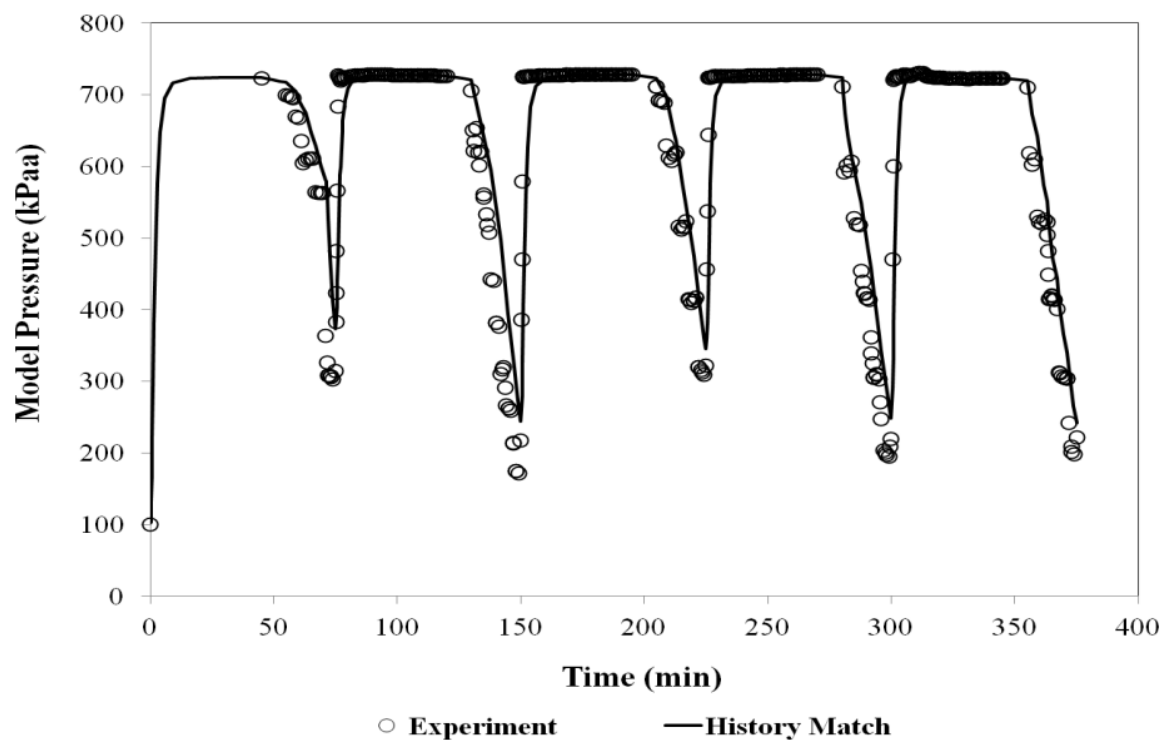

(b)

Figure 5-6 History matching results (a) production profiles (b) model pressure of Test $6(100 \mathrm{kPa} / \mathrm{min}$, center wormhole $)$ 


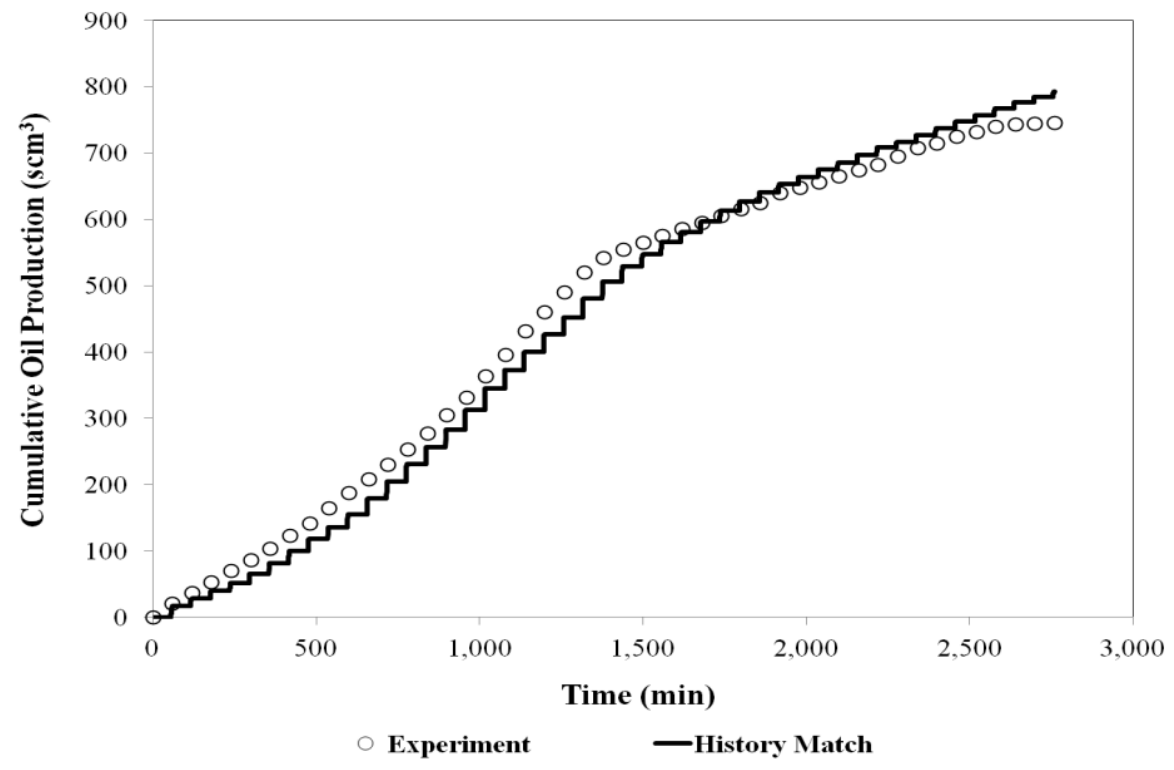

Figure 5-7 History matching results of Test 7 (500 kPa/5 min, center wormhole)

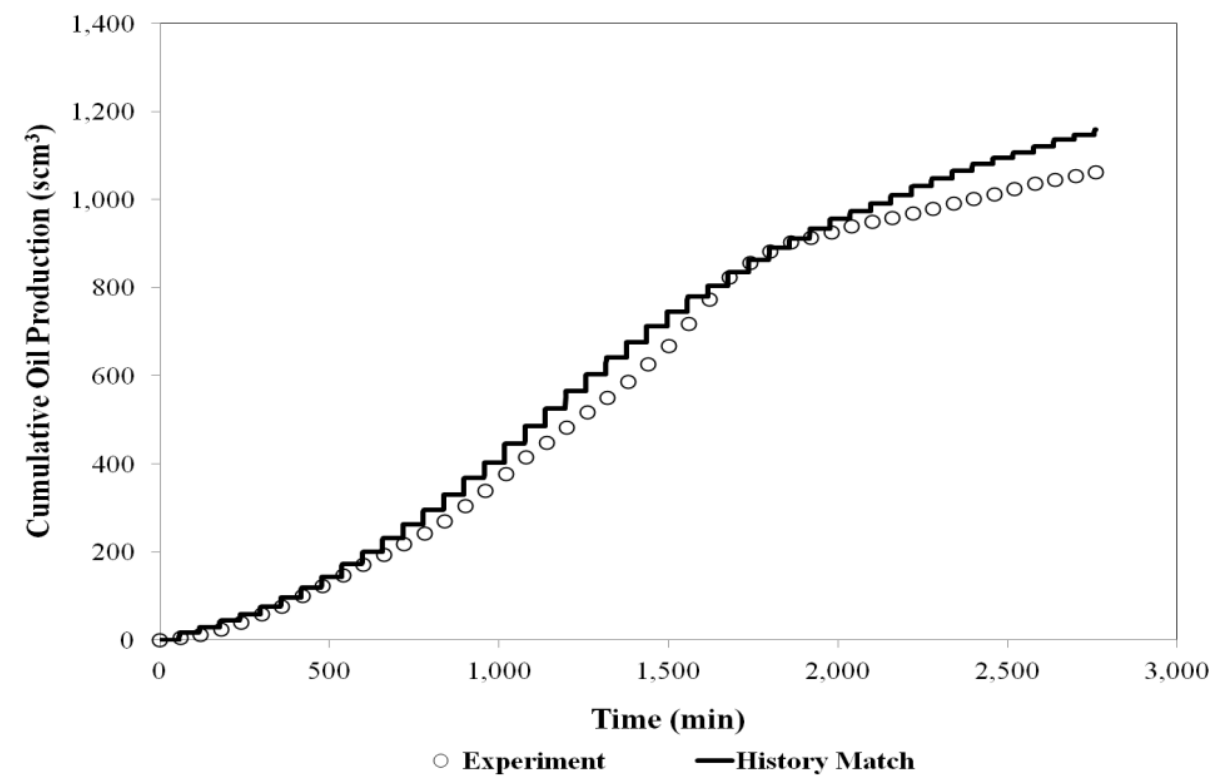

Figure 5-8 History matching results of Test $8(500 \mathrm{kPa} / 5 \mathrm{~min}$, bottom wormhole) 


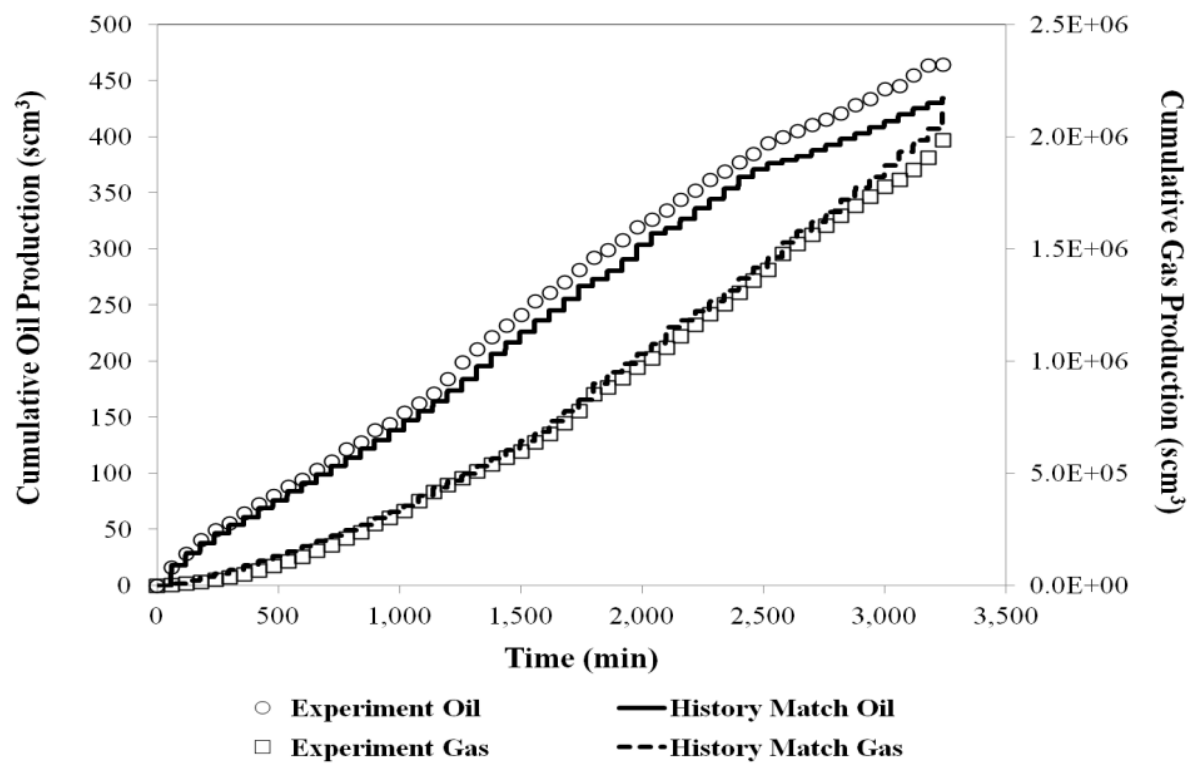

Figure 5-9 History matching results of Test $9(500 \mathrm{kPa} / 5 \mathrm{~min}$, top wormhole) 
The figures demonstrate that all CSI tests were successfully history-matched based on the modified fractional flow models. The deviations in the gas production profiles of Test 1 and 6 (Figure 5-1 and 5-6) were due to the faulty experimental measurements in the late cycles. The history-matching results of Test 7, 8, and 9 (Figure 5-7 to 5-9) are fairly reasonable in consideration of that the identical set of uncertain parameters were applied, including the gas-liquid relative permeability curves. In fact, these tests were individually evaluated, in which a very good agreement was yielded by slight changes in the gas-liquid curves in the injection stages. As a consequence, the capillary pressure curve between oil and gas phases (Figure 4-4) and dispersion coefficients of propane in oil phase $\left(0.0002 \mathrm{~cm}^{2} / \mathrm{min}\right)$ were validated as well as the gas-liquid relative permeability curves of Test 7.

\subsection{Tuned Gas-Liquid Relative Permeability Curves}

The tuned gas-liquid relative permeability curves are presented in Figure 5-10 to 5-16 in the order of the test number. For simplicity, the oil relative permeability in injection stages is denoted as $\mathrm{K}_{\text {rog_inj, }}$, the gas relative permeability in injection stages as $\mathrm{K}_{\mathrm{rg} \_ \text {inj }}$, the oil relative permeability in production stages as $\mathrm{K}_{\text {rog_prd}}$, and the gas relative permeability in production stages as $\mathrm{K}_{\mathrm{rg} \_ \text {prd. }}$ Appendix III presents the gas-liquid relative permeability tables for both injection and production stages. 


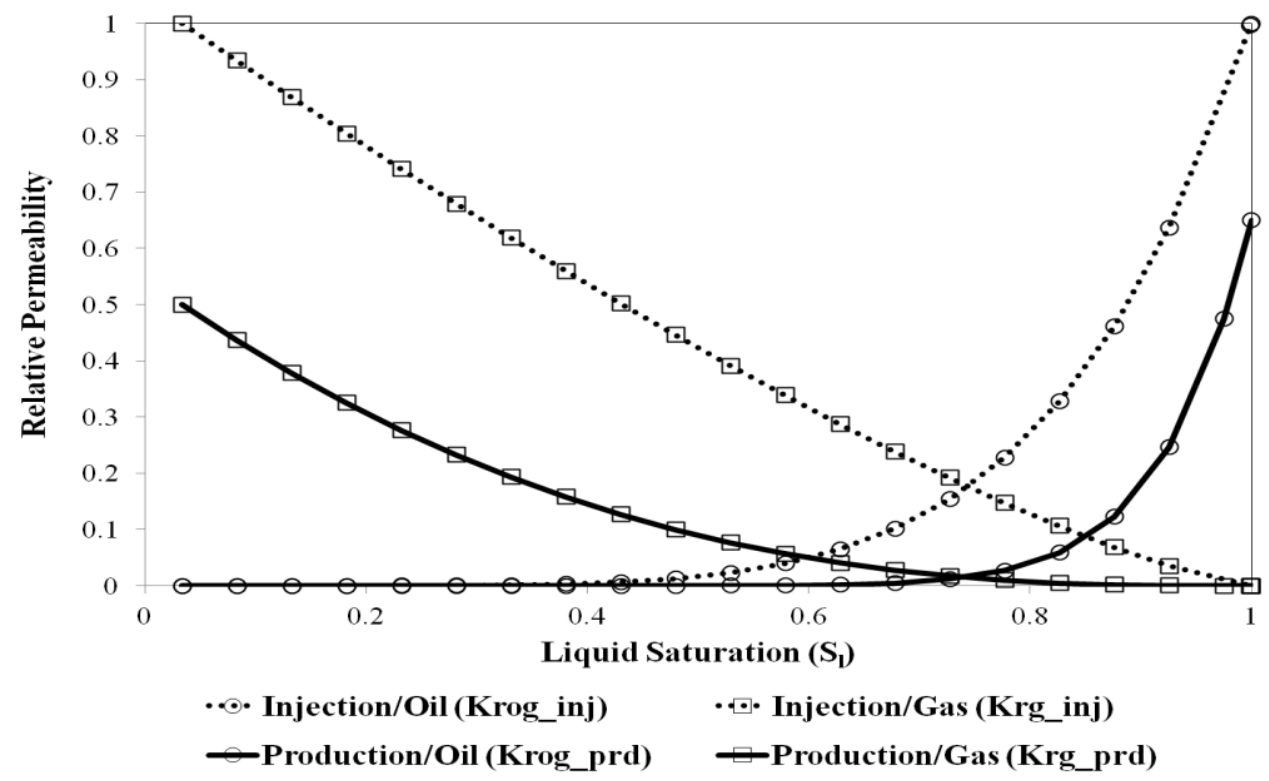

Figure 5-10 Tuned gas-liquid relative permeability curves of Test $1(1 \mathrm{kPa} / \mathrm{min})$

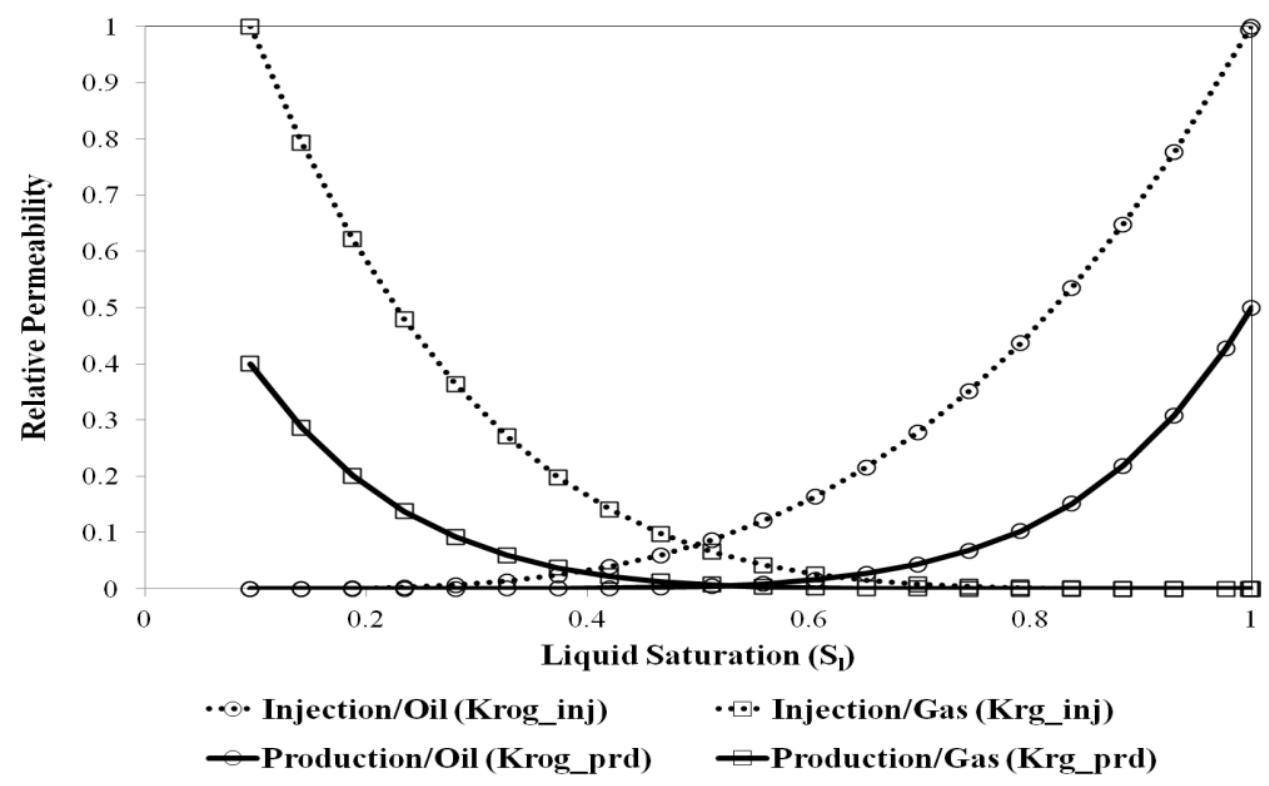

Figure 5-11 Tuned gas-liquid relative permeability curves of Test $2(3 \mathrm{kPa} / \mathrm{min})$ 


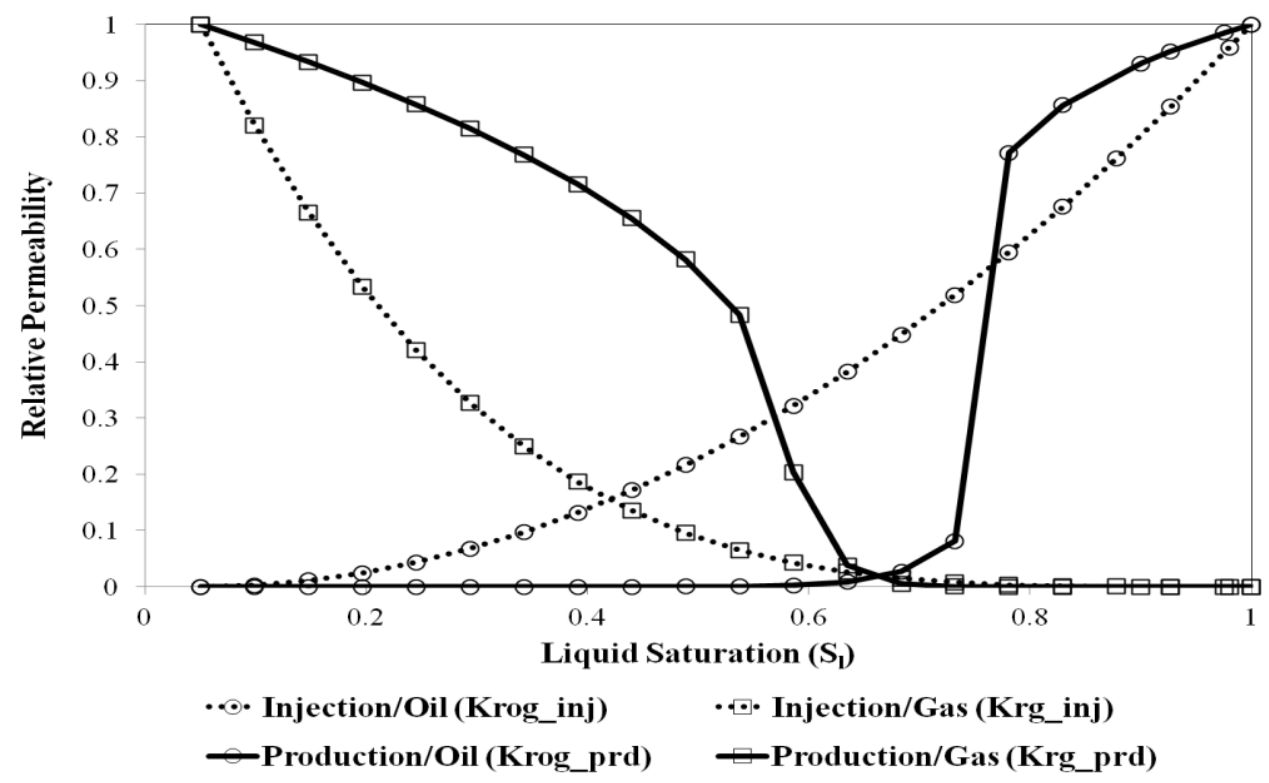

Figure 5-12 Tuned gas-liquid relative permeability curves of Test $3(5 \mathrm{kPa} / \mathrm{min})$

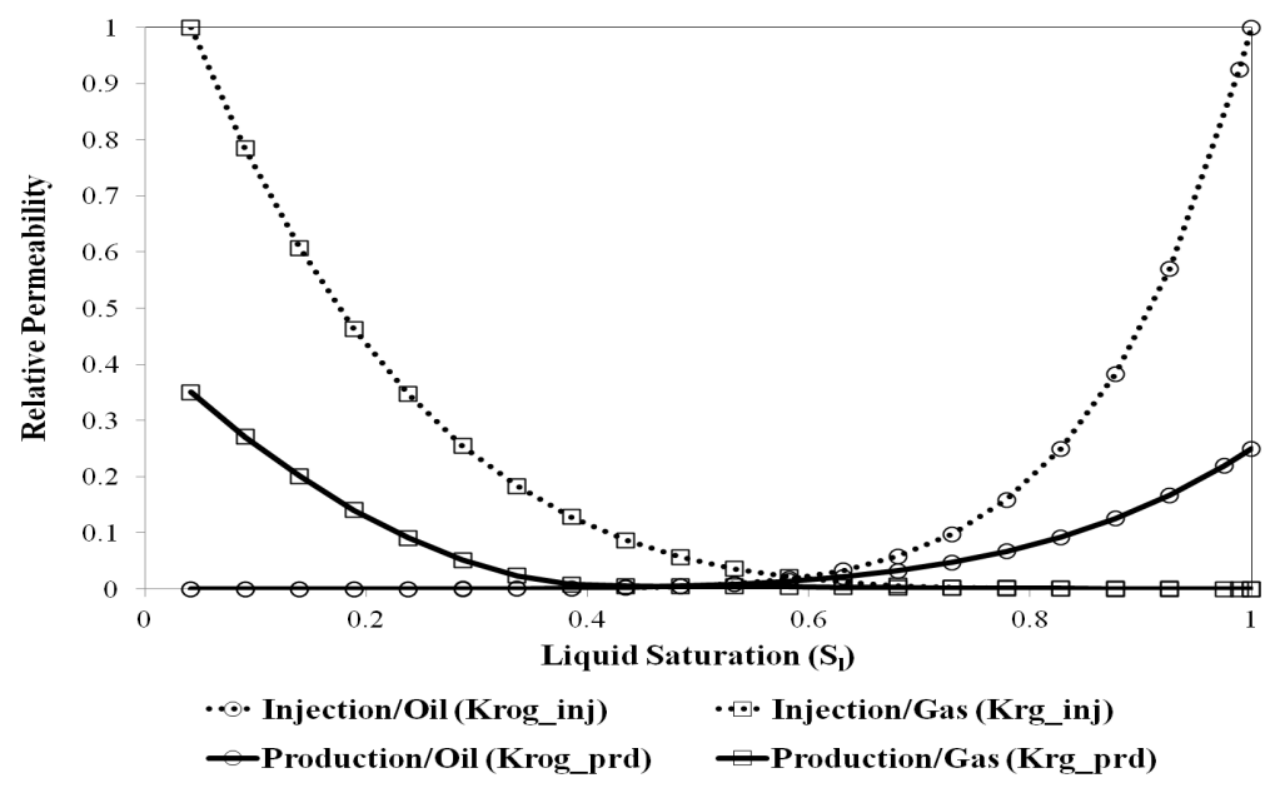

Figure 5-13 Tuned gas-liquid relative permeability curves of Test 4 (12.5

$\mathrm{kPa} / \mathrm{min})$ 


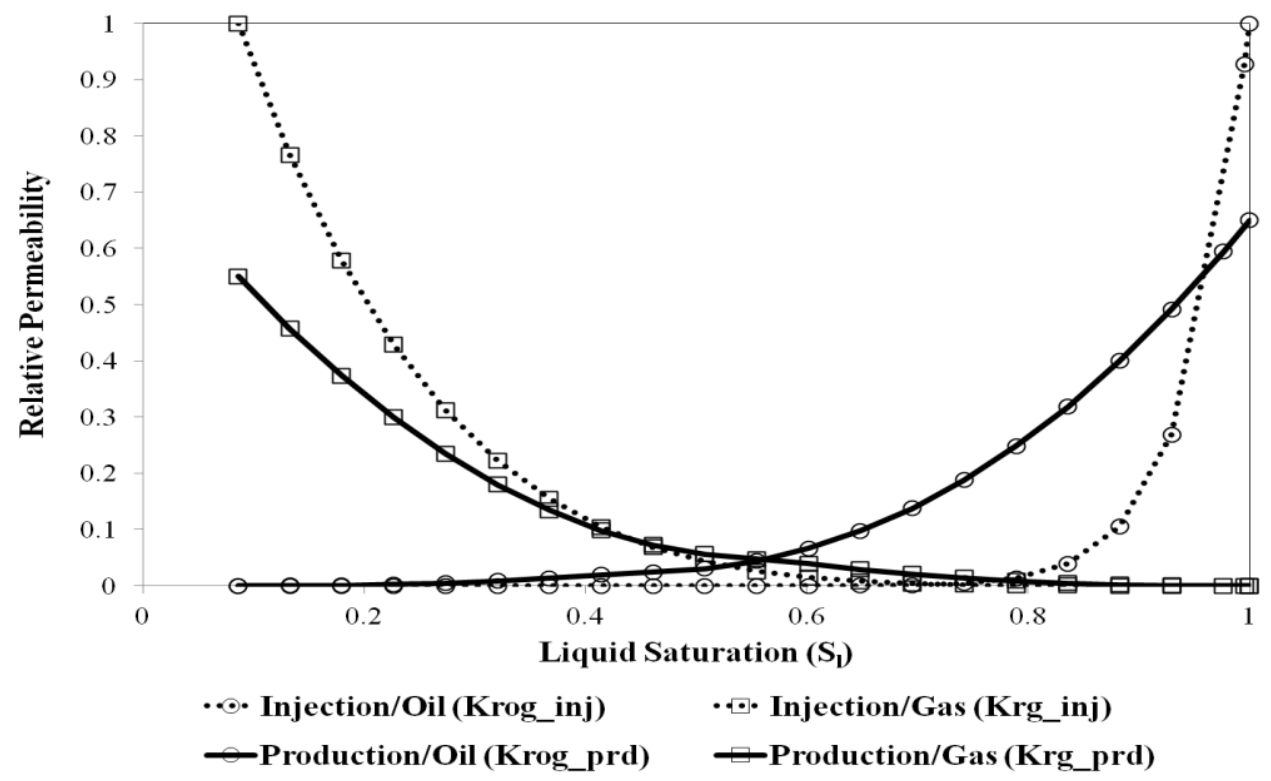

Figure 5-14 Tuned gas-liquid relative permeability curves of Test $5(50 \mathrm{kPa} / 4$ $\min )$

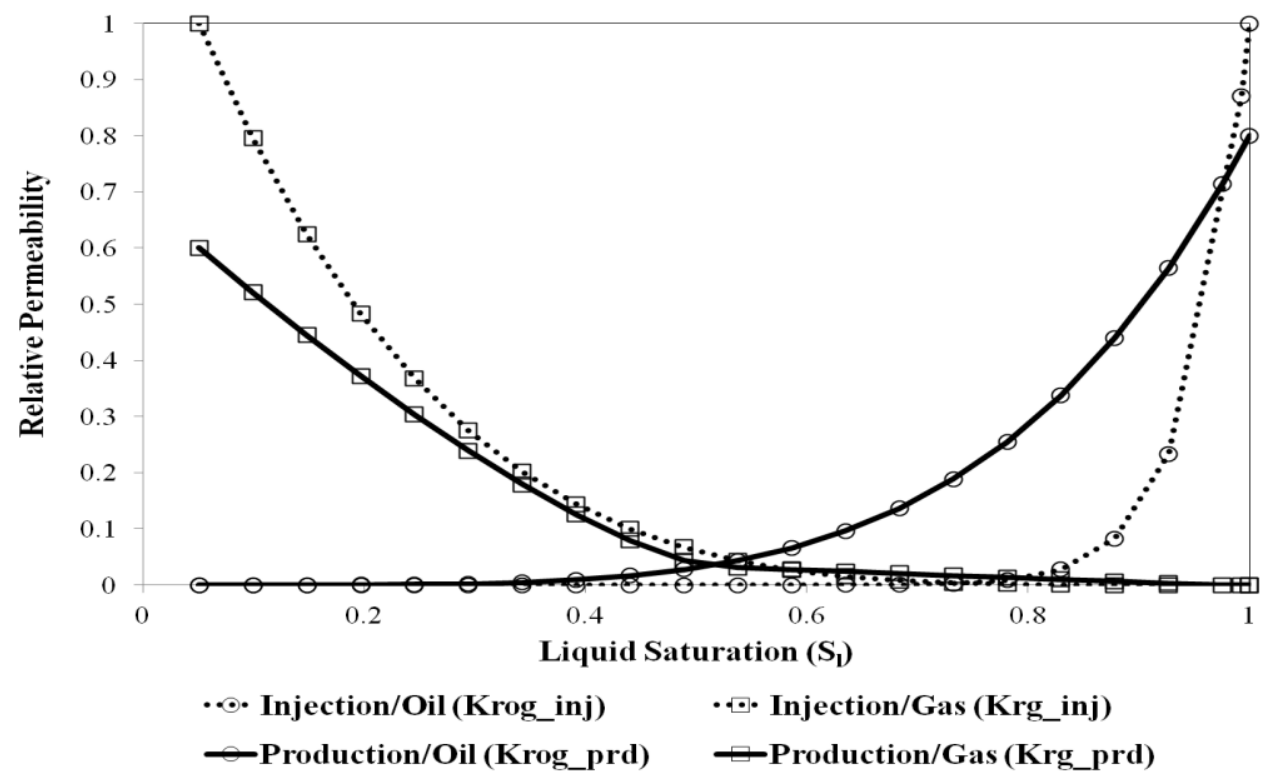

Figure 5-15 Tuned gas-liquid relative permeability curves of Test $6(100 \mathrm{kPa} / 4$ $\min )$ 


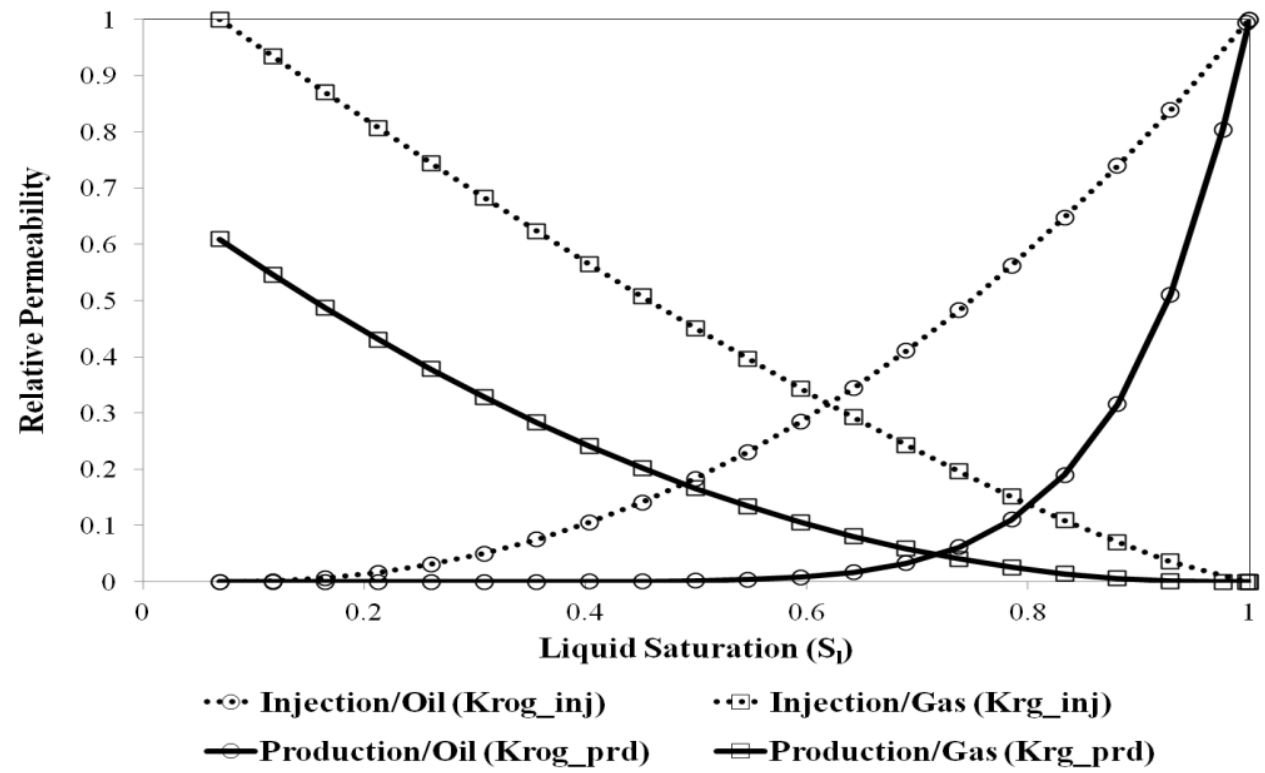

Figure 5-16 Tuned gas-liquid relative permeability curves of Test $7(500 \mathrm{kPa} / 5$ $\min )$ 


\section{Chapter 6. Discussions}

This chapter provides the discussions on the history-matching results as well as the tuned gas-liquid relative permeability curves. Firstly, the oil and gas production profiles are examined, which raises the need of explaining the solvent mass transfer mechanisms in CSI process. The tuned gas-liquid relative permeability curves are then analyzed at different pressure depletion rates and to identify the effect of the solvent chamber. Such analysis is performed in company with the sensitivity analysis on the tuned gas-liquid relative permeability curves. Furthermore, the results are compared to the other studies

introduced in the literature review. As a consequence, this chapter clearly demonstrates the unique characteristics of the gas-oil flow in CSI as well as the explanations on the phenomena responsible for the distinct behavior of the gasoil flow. The core points of the analysis and results are summarized at the end of each section.

\subsection{Oil and Gas Recovery}

The oil production profiles display that the oil recovery commences at low rates during a few or more beginning cycles, peaks thereafter, and gradually levels off. The low rates in the beginning indicate that the solvent, which facilitates the main recovery mechanisms of CSI, initially functions to a limited extent. The analysis of the oil production profiles therefore necessitates understanding of the 
solvent mass transfer mechanisms in CSI process and how these mechanisms progress with the recovery process.

In CSI, the solvent mass transfer mainly occurs through dissolution, diffusion, and convection. These mass transfer mechanisms are described as follows to clearly separate the routes through which the solvent spreads in oil phase. The dissolution takes place as the gaseous solvent penetrates into adjoining unsaturated oil. The dissolution rate is inversely determined by the solvent saturation in the oil phase. The diffusion and convection happen without phase change. The diffusion is driven solely by the concentration gradient and the convention by the potential difference. The convection refers to the mass transfer accompanied by a bulk movement of the fluid. Therefore, it is governed by the factors affecting the fluid flow in porous media. In CSI, the alternate injection and production changes the pressure gradient diametrically. Meanwhile, the concentration gradient lies in the same direction. Such tangled mixing directions exemplify the complexity in formulating the solvent distribution in a CSI process (Jia et al., 2015a). The relevant mathematical modeling still remains as a difficult challenge.

The initial conditions of CSI process are unfavorable for the solvent mass transfer mechanisms. The untapped and thus thick oil phase causes a large denominator in the concentration gradient. Furthermore, the low gas voidage only allows limited vacant spaces available for the in-situ fluids to migrate. As a 
result, the diffusion is hindered by the poor molecular driving force. The convection also feebly occurs due to the spatial restrictions. In heavy oil systems, the dissolution is decelerated in achieving an equilibrium solvent concentration (Tharanivasan et al., 2004). Such delay in saturating the oil with the solvent reflects the time and space needed for the solvent to take effect in the recovery process.

With repeated CSI cycles, the mixing between oil and solvent eventually undergoes temporal and spatial improvements. The solvent molecules are given the time to permeate into the oil phase and spread towards the untouched area. The produced heavy oil in the interim gives away vacant spaces. The widened bulk movements of the in-situ fluids then further advances the solvent dispersal under the repeated huff-n-puff operation. If the solvent possesses a high solubility, such as propane, the oil phase more likely attains the equilibrium solvent concentration towards the end of the process.

Provided that the mixing between oil and solvent progresses with repeated cycles, the oil production profiles can be explained. The low rates in the beginning cycles are due to the abysmal solvent mass transfer. The solvent mass transfer initially functions to a limited extent and thus insufficiently facilitates the main recovery mechanisms of CSI. The oil rate then accelerates to the peak after a few or more cycles in accordance with the improving solvent mass transfer. Meanwhile, the solvent chamber grows in size but not as much to decelerate the 
oil recovery. The oil production profile eventually approaches horizontality as the growing solvent chamber yields the adverse effects prevailing over the solvent benefits. The solvent chamber is disadvantageous to the oil recovery as it promotes the free gas discharge and thus the in-situ pressure dissipation. It also serves as porous resistance to the oil flow and a high gas saturation zone abating the fractional flow of the oil. These adverse effects of the solvent chamber remain dominant even if the dissolved solvent achieves the systemwide phase equilibrium.

The delayed peak production was also observed in the other studies of CSI (Ivory et al., 2009; Chang et al., 2013; Jia et al., 2015b). This phenomenon however disagrees with the recovery tendency of Vapor Extraction (VAPEX). In VAPEX, the maximum oil rate appears in the very first stage. Such dominant early production is a result of the optimized gravity drainage during the chamber rising phase (Das et al., 1998). The inconsistency between CSI and VAPEX therefore suggests that in CSI process the gravity force plays an inessential role as a drive mechanism.

The gas production profiles show rather a typical trend of solution gas drive. The cumulative gas production increases slowly in the beginning but rises after a certain period. The gas rates are low in the early cycles because the in-situ gas saturation is initially low and thus barely exceeds the critical gas saturation. 
After a certain number of cycles, the gas rates become higher and increase continuously as the in-situ gas saturation increases.

The cumulative producing GOR and recovery factor have been widely utilized for the evaluation of the changing nature of the foamy oil flow at different pressure depletion rates. Table 6-1 and 6-2 tabulate these oil and gas recovery properties obtained from the CSI tests. 
Table 6-1 Cumulative producing GOR and recovery factor

\begin{tabular}{|c|c|c|c|c|}
\hline $\begin{array}{c}\text { Test } \\
\#\end{array}$ & $\begin{array}{c}\text { Pressure } \\
\text { Depletion } \\
\text { Rate }\end{array}$ & $\begin{array}{c}\text { Pressure } \\
\text { Depletion Rate } \\
\text { Classification }\end{array}$ & $\begin{array}{c}\text { Cum. Oil } \\
\text { Produced } \\
\left(\mathrm{scm}^{3}\right)\end{array}$ & $\begin{array}{c}\text { Cum. Gas } \\
\text { Produced } \\
\left(10^{3} \mathrm{scm}^{3}\right)\end{array}$ \\
\hline 1 & $1 \mathrm{kPa} / \mathrm{min}$ & Lowest & 921 & 1,768 \\
\hline 2 & $3 \mathrm{kPa} / \mathrm{min}$ & Very Low & 1,061 & 2,522 \\
\hline 3 & $5 \mathrm{kPa} / \mathrm{min}$ & Low & 875 & 2,290 \\
\hline 4 & $12.5 \mathrm{kPa} / \mathrm{min}$ & Middle & 1,244 & 6,066 \\
\hline 5 & $50 \mathrm{kPa} / 4 \mathrm{~min}$ & High & 1,024 & 6,276 \\
\hline 6 & $100 \mathrm{kPa} / 4 \mathrm{~min}$ & Very High & 1,026 & 5,403 \\
\hline 7 & $500 \mathrm{kPa} / 5 \mathrm{~min}$ & Highest & 745 & - \\
\hline
\end{tabular}

Table 6-2 Cumulative producing GOR and recovery factor

\begin{tabular}{|c|c|c|c|c|}
\hline $\begin{array}{c}\text { Test } \\
\#\end{array}$ & $\begin{array}{c}\text { Pressure } \\
\text { Depletion } \\
\text { Rate }\end{array}$ & $\begin{array}{c}\text { Pressure } \\
\text { Depletion Rate } \\
\text { Classification }\end{array}$ & $\begin{array}{c}\text { Cum. } \\
\text { Producing GOR } \\
\left(10^{3} \mathrm{scm}^{3} / \mathrm{scm}^{3}\right)\end{array}$ & $\begin{array}{c}\text { Recovery } \\
\text { Factor } \\
(\%)\end{array}$ \\
\hline 1 & $1 \mathrm{kPa} / \mathrm{min}$ & Lowest & 1.92 & 50 \\
\hline 2 & $3 \mathrm{kPa} / \mathrm{min}$ & Very Low & 2.38 & 59 \\
\hline 3 & $5 \mathrm{kPa} / \mathrm{min}$ & Low & 2.62 & 49 \\
\hline 4 & $12.5 \mathrm{kPa} / \mathrm{min}$ & Middle & 4.88 & 68 \\
\hline 5 & $50 \mathrm{kPa} / 4 \mathrm{~min}$ & High & 6.13 & 60 \\
\hline 6 & $100 \mathrm{kPa} / 4 \mathrm{~min}$ & Very High & 5.27 & 57 \\
\hline 7 & $500 \mathrm{kPa} / 5 \mathrm{~min}$ & Highest & - & 43 \\
\hline
\end{tabular}


The tables show that the cumulative producing GOR as well as the recovery factor noticeably vary with the pressure depletion rate. The cumulative producing GOR ranges between $1.92 * 10^{3}$ and $6.13 * 10^{3} \mathrm{scm} / \mathrm{scm}$ and the recovery factor between 43 and $68 \%$. Such considerable variances indicate that the gas-oil flow is greatly affected by the pressure depletion rate.

However, a direct comparison between the tests is very problematic and thus impractical. The problems arise because the pressure depletion rate is in a pair relationship with the operational parameters that are irrelevant or indirectly related to the foamy oil flow and yet alter the recovery process. A conclusive example is the number of cycles and the duration of recovery process. These parameters are irrelevant or indirectly related to the foamy oil flow. Nonetheless, the number of cycles still affects the producing GOR since it determines the gross quantity of the free gas that merely fills and vacates the solvent chamber without phase change. Moreover, the number of cycles and the process time directly impact the recovery factor as these parameters are coupled with the solvent mass transfer mechanisms and thus the overall mixing between oil and solvent. The number of cycles corresponds to the frequency of the in-situ fluids agitation (i.e. convection). The process time represents the time given for the solvent to permeate and spread in the oil phase (i.e. dissolution and diffusion).

Consequently, in order to truly reflect the effect of the pressure depletion rate, it is necessary to remove the consequences of the operational parameters that are 
not or indirectly related to the foamy oil nature. Such analytical approach is very complicated and thus impractical.

In summary, the analysis of the oil and gas production profiles shows that the gas-oil flow in CSI greatly varies with the pressure depletion rate. However, the oil and gas production profiles do not truly reflect the effect of the pressure depletion rate on the foamy oil nature in CSI process. This is because the pressure depletion rate is in a pair relationship with the operational parameters (i.e. number of cycles and duration of recovery process) that alter the recovery process regardless of the foamy oil flow. Therefore, a thorough analysis of the gas-oil flow in CSI further requires examining the gas-liquid relative permeability curves which solely involve the characteristics of the gas-oil flow.

\subsection{Gas-Liquid Relative Permeability Curves for Production}

\section{Stages}

The gas-liquid relative permeability curves serve as a direct means of analyzing the gas-oil flow, in which the characteristics of the two-phase flow are more thoroughly reflected. The gas relative permeability curve in production stages is in particular importance as being under the greater influence of the foamy oil flow. In this study, a hypothetical property, named the low mobility gas saturation, is added to quantify the lowness of the gas relative permeability curves. It is defined as the gas saturation below which the gas relative permeability is lower than 0.01 . 
Table 6-3 Summary of tuned gas relative permeability curves in production stages

\begin{tabular}{|c|c|c|c|c|c|}
\hline $\begin{array}{c}\text { Test } \\
\#\end{array}$ & $\begin{array}{c}\text { Pressure } \\
\text { Depletion } \\
\text { Rate }\end{array}$ & $\begin{array}{c}\text { Pressure } \\
\text { Depletion Rate } \\
\text { Classification }\end{array}$ & $\begin{array}{c}\text { Critical Gas } \\
\text { Saturation } \\
(\%)\end{array}$ & $\begin{array}{c}\text { Low Mobility } \\
\text { Gas Saturation } \\
(\%)\end{array}$ & $\begin{array}{c}\text { End } \\
\text { Point }\end{array}$ \\
\hline 1 & $1 \mathrm{kPa} / \mathrm{min}$ & Lowest & 3 & 22 & 0.50 \\
\hline 2 & $3 \mathrm{kPa} / \mathrm{min}$ & Very Low & 7 & 51 & 0.40 \\
\hline 3 & $5 \mathrm{kPa} / \mathrm{min}$ & Low & 10 & 32 & 1 \\
\hline 4 & $12.5 \mathrm{kPa} / \mathrm{min}$ & Middle & 3 & 61 & 0.35 \\
\hline 5 & $50 \mathrm{kPa} / 4 \mathrm{~min}$ & High & 3 & 21 & 0.55 \\
\hline 6 & $100 \mathrm{kPa} / 4 \mathrm{~min}$ & Very High & 3 & 15 & 0.60 \\
\hline 7 & $500 \mathrm{kPa} / 5 \mathrm{~min}$ & Highest & 3 & 14 & 0.60 \\
\hline
\end{tabular}


The properties of the tuned gas relative permeability curves in the production stages $\left(\mathrm{K}_{\mathrm{rg} \_ \text {prd }}\right.$ curves) are summarized in Table 6-3.

\subsubsection{Effect of Pressure Depletion Rate}

Table 6-3 illustrates that the critical gas saturation increases with the pressure depletion rate from $3 \%$ at $1 \mathrm{kPa} / \mathrm{min}$ to $10 \%$ at $5 \mathrm{kPa} / \mathrm{min}$. It then drops abruptly to $3 \%$ at the next high depletion rate, $12.5 \mathrm{kPa} / \mathrm{min}$, and further remains constant up to $500 \mathrm{kPa} / 5 \mathrm{~min}$. The critical gas saturation therefore ranges from the lowest (3\%) to the highest value (10\%) when the depletion rate is low (Test 1 to 3 ). It however suddenly plateaus at the lowest value as the depletion rate further increases (Test 4 to 7 ).

The increasing trend of the critical gas saturation at the low pressure depletion rates agrees with the findings of Ostos et al. (2004), whereas the trend beyond that point is in conflict. In this study, the critical gas saturation drops to and plateaus at the lowest value, while according to Ostos et al. (2004) should remain constant at the highest level. Ostos et al. (2004) reported that the critical gas saturation increases with the pressure depletion rate until a maximum attainable value. However, they further claimed that the critical gas saturation plateaus at the highest level at higher depletion rates instead of appearing low.

In this study, to justify the unlike behavior of the critical gas saturation, the history-matching results of Test 4 to 7 were first validated with the additional simulation runs. The critical gas saturations in these runs were increased to $10 \%$ 
as was the maximum value obtained from Test 3 . The additional runs thereby complied with the findings of Ostos et al. (2004) and by validating the historymatching results justified the low critical gas saturations. The dominating differences between this study and the study of Ostos et al. (2004) were then analyzed to investigate the phenomena responsible for the abrupt drop and low values of the critical gas saturation.

Figure 6-1 to 6-4 present the results of the additional runs in comparison with the history-matching results. 


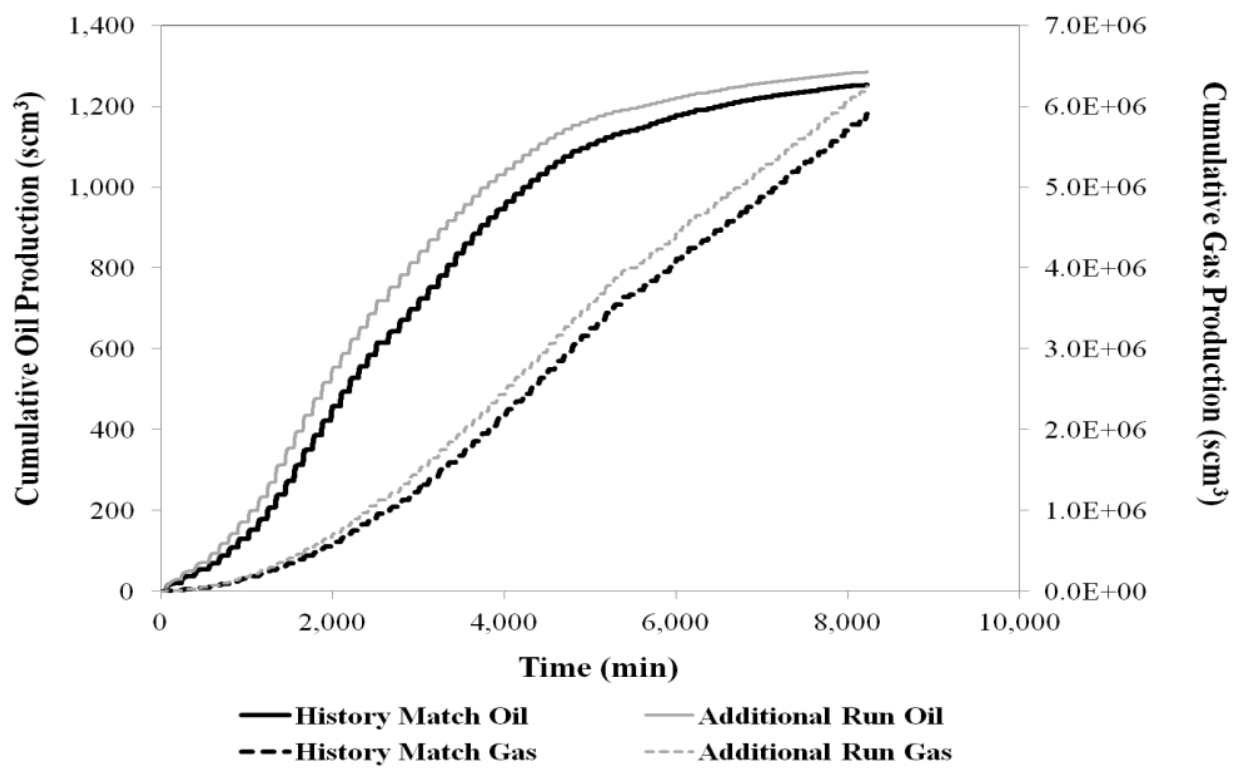

(a)

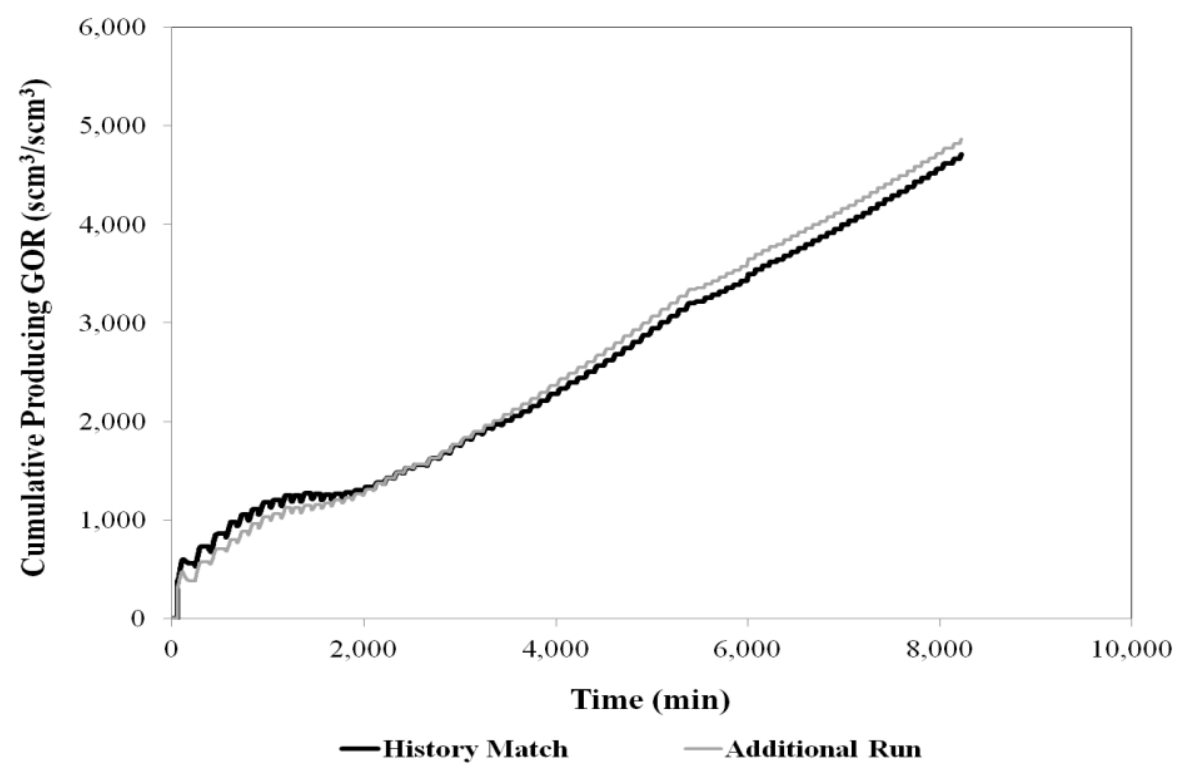

(b)

Figure 6-1 Additional run with increased $S_{\text {gcrit }}($ a) cumulative oil and gas productions (b) cumulative producing GOR (Test 4) 


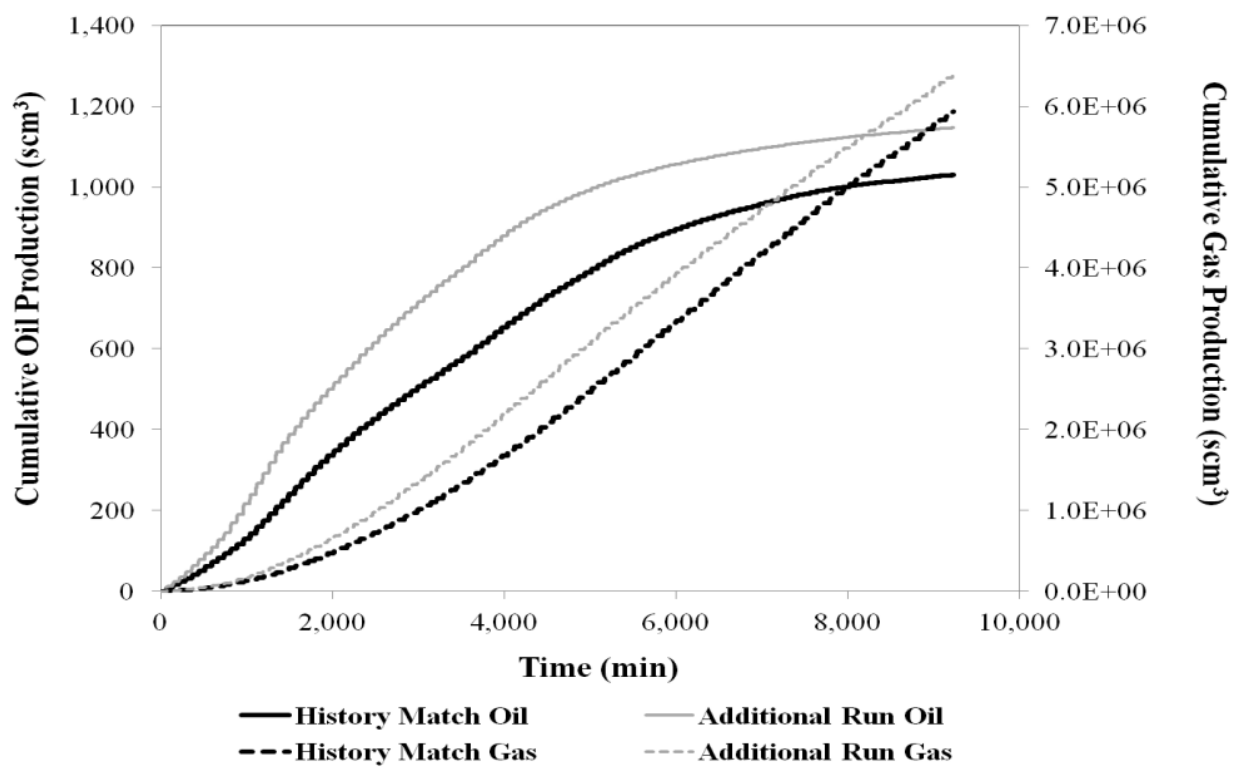

(a)

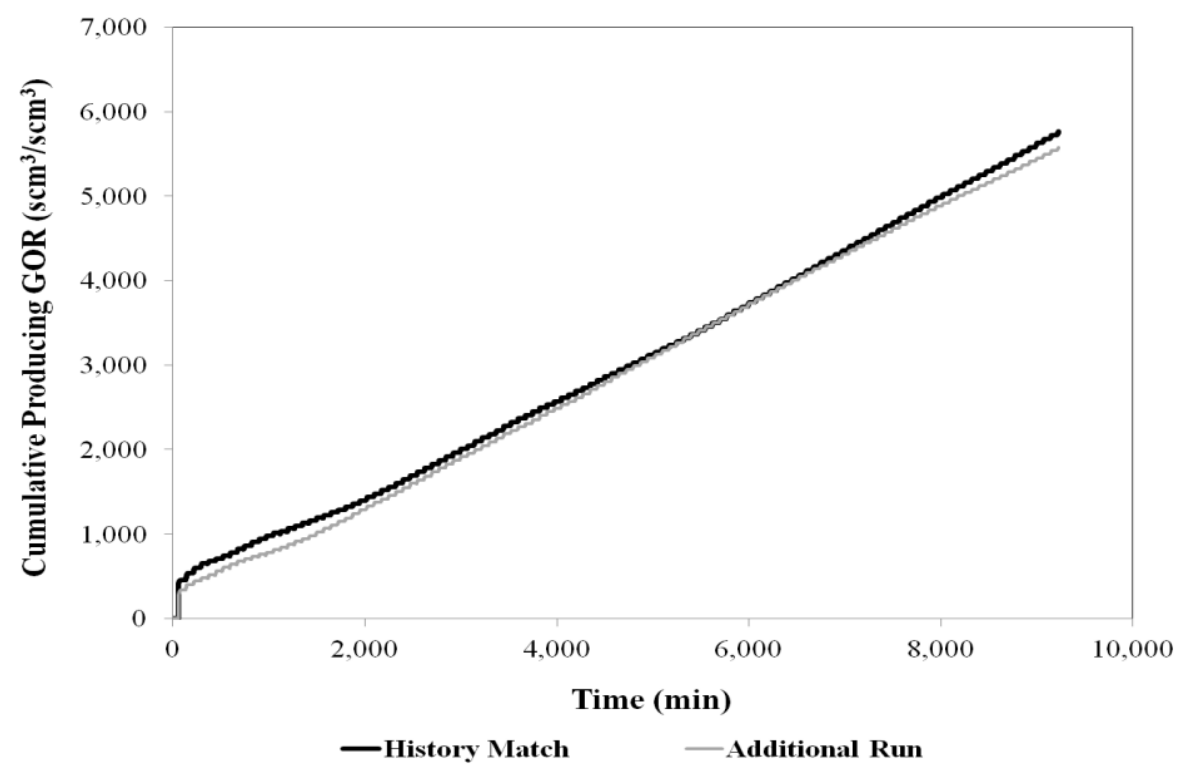

(b)

Figure 6-2 Additional run with increased $S_{\text {gcrit }}($ a) cumulative oil and gas productions (b) cumulative producing GOR (Test 5) 


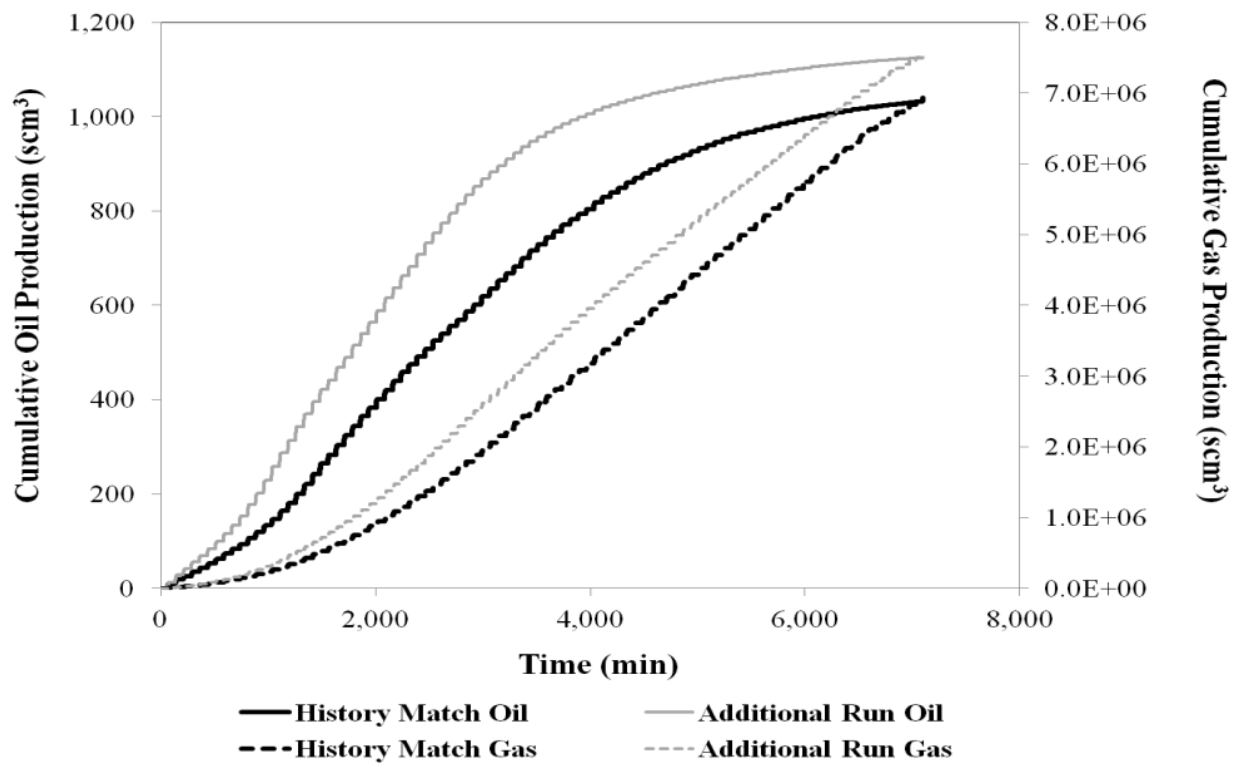

(a)

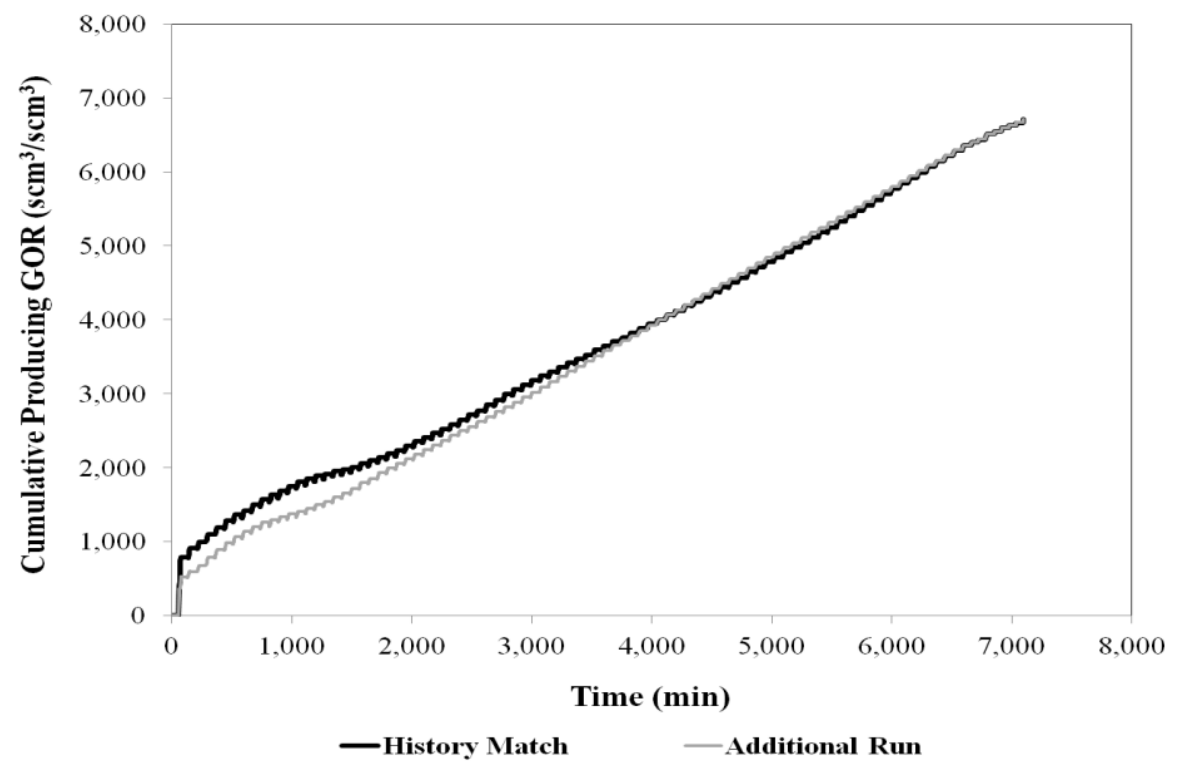

(b)

Figure 6-3 Additional run with increased $S_{\text {gcrit }}($ a) cumulative oil and gas productions (b) cumulative producing GOR (Test 6) 


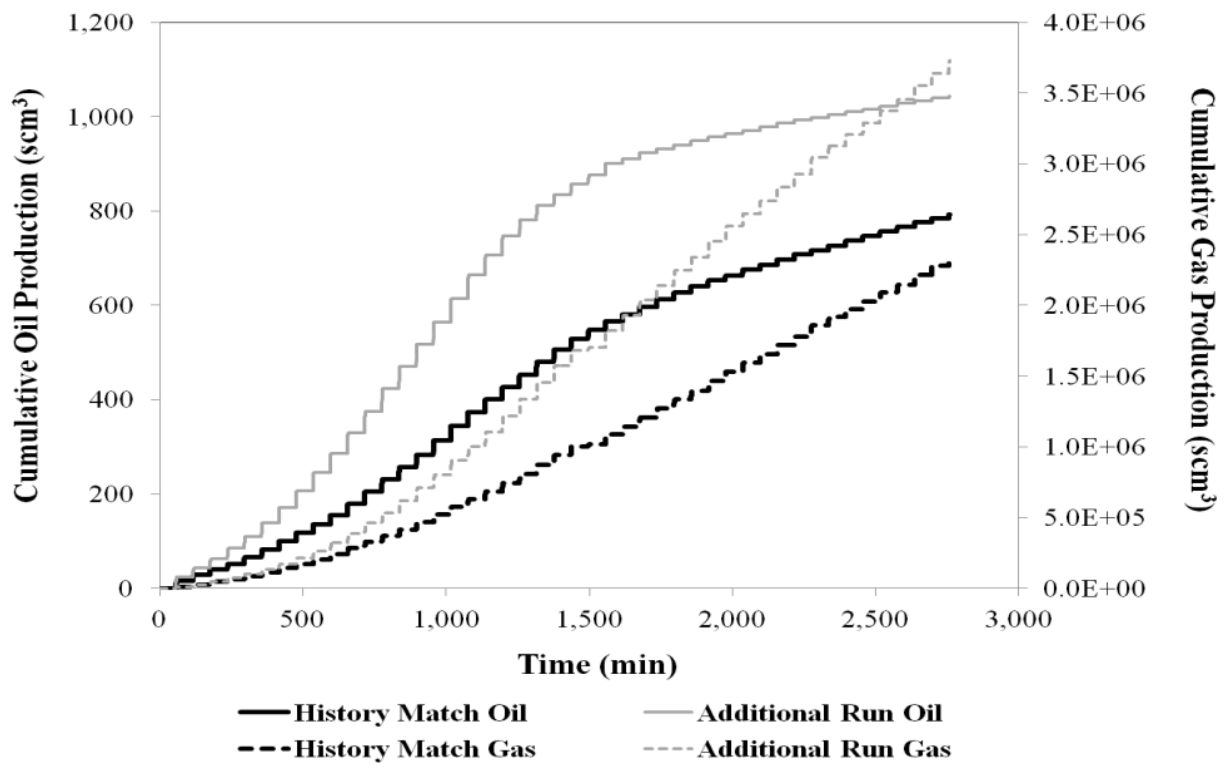

(a)

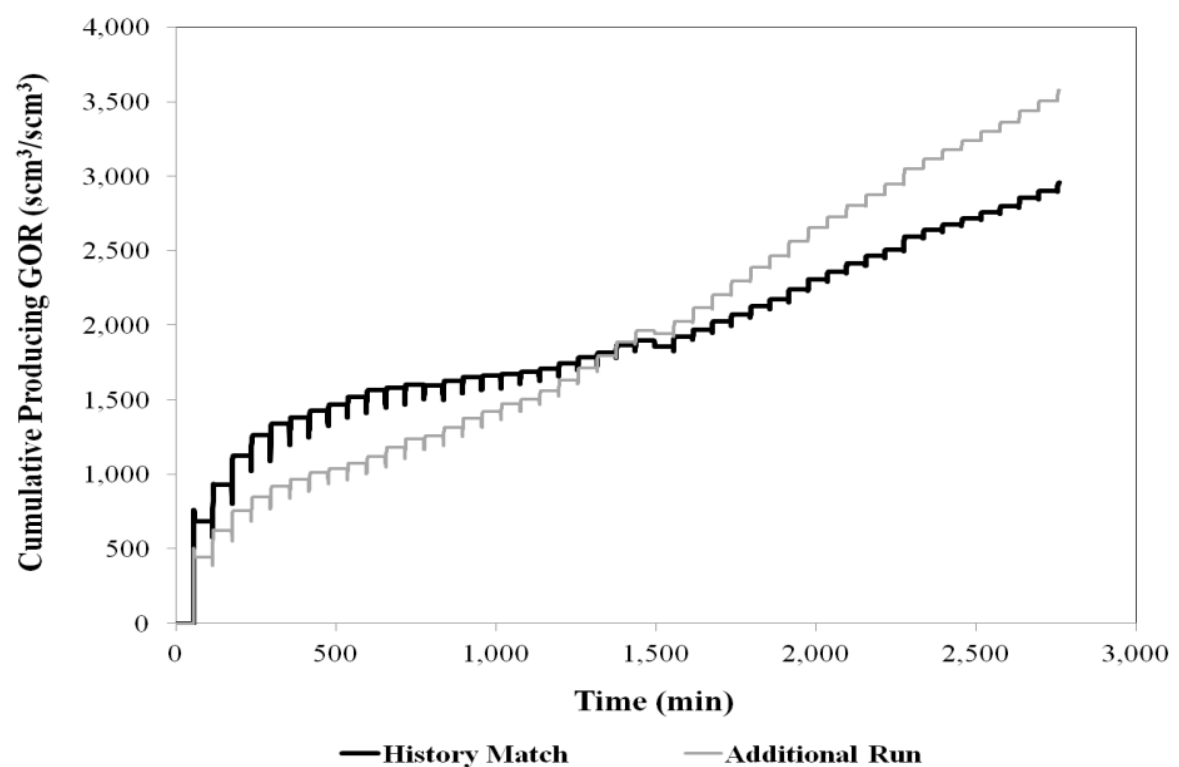

(b)

Figure 6-4 Additional run with increased $S_{\text {gcrit }}($ a) cumulative oil and gas productions (b) cumulative producing GOR (Test 7) 
The figures show that the additional simulation runs yielded noticeable deviations in the cumulative oil and gas productions as well as the cumulative producing GOR. This reflects that the recovery process is highly sensitive to the critical gas saturation. The low values of the critical gas saturation are therefore validated as these values yielded the successful history-matching results. In Test 4 , due to the very slowly increasing gas relative permeability, the increase of the critical gas saturation only required reducing the gas relative permeability by maximum $4 * 10^{-4}$. The recovery factor nonetheless improved unexpectedly high about $2 \%$ at the end cycle and even greater in the earlier cycles. In general, the higher critical gas saturation increased both oil and gas productions but decreased the producing GOR. As exceptions, the cumulative producing GOR appears higher in the late cycles of Test 4 and 7. The reduced producing GOR indicates that the solution gas, rather than free gas, mainly accounts for the increase in the gas production. The higher cumulative producing GOR in the late cycles more likely implies that the increase in the cumulative oil production develops a bigger solvent chamber promoting the free gas flow.

It has been provided that the low critical gas saturations are imperative and unique. Therefore, the dominating differences between this study and the study of Ostos et al. (2004) explain the phenomena responsible for the abrupt drop and low level of the critical gas saturation. The differences appear in the recovery process, component of solution gas, and range of the pressure depletion rate, which are tabulated in Table 6-4. 
Table 6-4 Dominating differences between this study and Ostos et al. (2004)

\begin{tabular}{|c|c|c|}
\hline Differences & This study & Ostos et al. (2004) \\
\hline Recovery Process & CSI & Heavy Oil Solution Gas Drive \\
\hline Solution Gas & Propane & Methane \\
\hline Pressure Depletion Rate & $1 \mathrm{kPa} / \mathrm{min} \sim 500 \mathrm{kPa} / 5 \mathrm{~min}$ & $0.2827 \sim 2.2545 \mathrm{kPa} / \mathrm{min}$ \\
\hline
\end{tabular}


As previously discussed, the gas-oil flow in CSI considerably differs from that in heavy oil solution gas drive. The differences mainly arise from the free gas in CSI, which originates from the solvent chamber. The free gas that originates from the solvent chamber is independent of the pseudobubblepoint pressure of oil. As a result, in CSI, the free gas exists even when the dispersed gas bubbles are immobile. Moreover, it eventually adjoins and by cohesion draws the gas bubbles into the continuous gas phase at a sufficiently high pressure depletion rate. A rapid pressure drop puts the free gas under turbulence and also promotes the nucleation of the dispersed gas bubbles. When the turbulence is strong enough, the free gas happens to come in contact with the dispersed gas bubbles. The more the gas bubbles nucleate, the higher the probability is for the free gas to contact the gas bubbles. Consequently, the dispersed gas bubbles merge into the continuous gas phase. During such process, the gas bubbles gain mobility, including the ones that were immobile at a lower depletion rate. The critical gas saturation drops simultaneously and remains low even if the pressure depletion rate further increases.

Propane is highly soluble than methane, yielding a greater amount of the dissolved gas in oil phase. Therefore, in propane-based systems, the dispersed gas bubbles are more heavily populated in the pore bodies. As a result, the free gas has a higher probability to adjoin the gas bubbles and to result in the low critical gas saturation. 
The pressure depletion rates in this study are one or more order of magnitude higher than those in the study of Ostos et al. (2004). The considerably higher pressure depletion rates may have caused the different behavior of the critical gas saturation. However, the argument based on the range of the depletion rate necessitates the analysis that is complex and thus impractical. It requires comparing the pressure propagation in the different systems. The pressure propagation is a function of complexly related well and/or rock properties (i.e. well geometry, dimensions of porous medium, rock permeability, etc.)

In addition to the critical gas saturation, the low mobility gas saturation also exhibits the effect of the pressure depletion rate on the gas relative permeability curve. In this study, the low mobility gas saturation is defined as the gas saturation below which the gas relative permeability is lower than 0.01 . It therefore reflects how low the gas relative permeability evolves. Figure 6-5 displays the low mobility gas saturation changing with the pressure depletion rate as well as the critical gas saturation. 


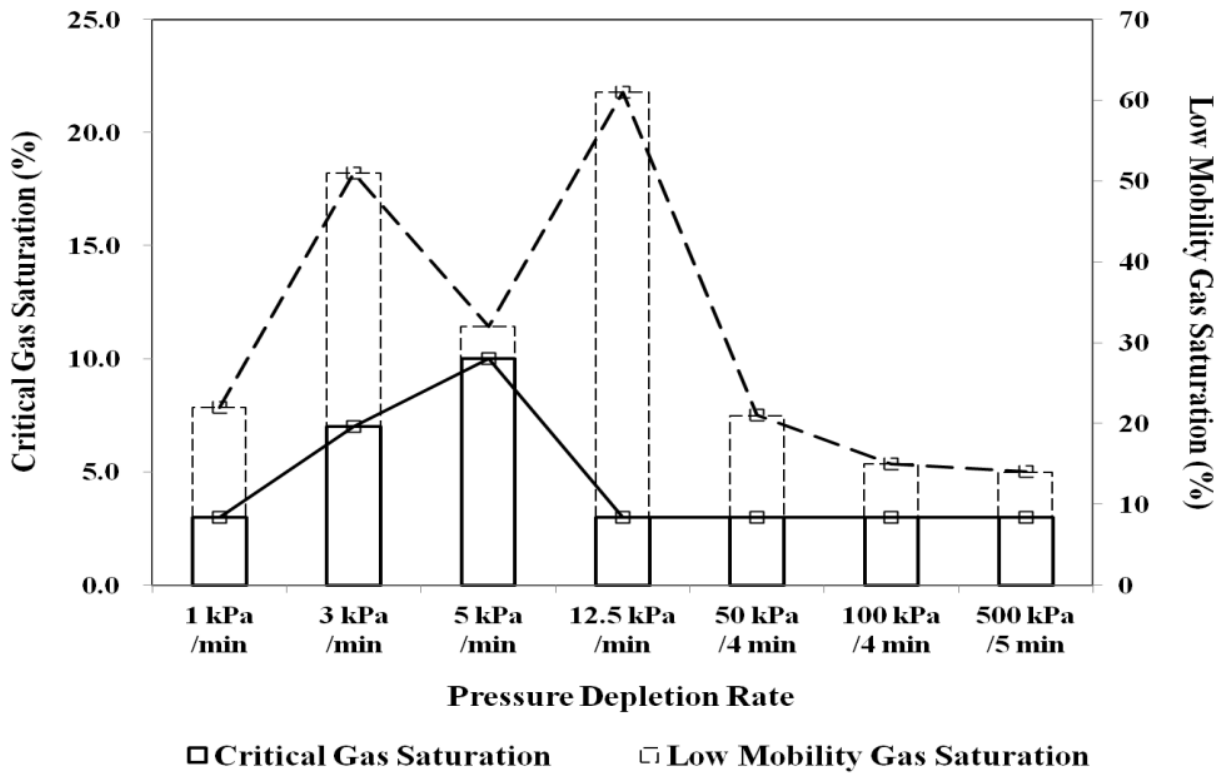

Figure 6-5 Critical gas saturation and low mobility gas saturation 
The figure shows that the low mobility gas saturation in general increases with the pressure depletion rate when the depletion rate ranges from 1 to $12.5 \mathrm{kPa} / \mathrm{min}$. With the further increase in the pressure depletion rate, it however decreases and nearly plateaus at $100 \mathrm{kPa} / \mathrm{min}$. The disagreeing trend at $5 \mathrm{kPa} / \mathrm{min}$ should be neglected because the corresponding gas-liquid relative permeability curves were misled into the distinctive shapes (Figure 5-12) due to the effect of the solvent chamber. The solvent chamber significantly affects the gas-liquid relative permeability curves involving a high critical gas saturation as will be discussed in the following section.

The critical gas and low mobility gas saturations together describe the general characteristics of the gas relative permeability curve. Figure 6-5 illustrates that these two parameters characterize the gas relative permeability curves differently at the low, middle, and high pressure depletion rates.

At the low pressure depletion rates (i.e. $1 \sim 5 \mathrm{kPa} / \mathrm{min}$ ), both critical gas and low mobility gas saturations increase with the depletion rate. In other words, a higher critical gas saturation eventuates in lower gas relative permeabilities. Such trend is natural from the perspective of mobility. A higher critical gas saturation is equivalent to an extended immobility of the gas phase. In fact, it also appears in the study of Ostos et al. (2004) and is recognized effect of the pressure depletion rate on the foamy oil flow in heavy oil solution gas drive. 
At the middle pressure depletion rate (i.e. $12.5 \mathrm{kPa} / \mathrm{min}$ ), the critical gas saturation drops to the lowest value $(3 \%)$ while the low mobility gas saturation increases to the highest level. As previously discussed, the critical gas saturation decreases due to the phenomena that the free gas adjoins and draws the immobile gas bubbles into the continuous gas phase. The depletion rate is therefore sufficiently high that the gas bubbles are mobilized in such ways. However, it is not high enough to improve the gas phase mobility. The gas bubbles that have become mobile initially gain a substantially low mobility which but increases at higher depletion rates.

At the high pressure depletion rates (i.e. $50 \mathrm{kPa} / 4 \mathrm{~min} \sim 500 \mathrm{kPa} / 5 \mathrm{~min}$ ), the critical gas saturation remains constant at the lowest value, while the low mobility gas saturation decreases with the depletion rate and eventually plateaus. This reflects that the decrease in critical gas saturation brings the phenomenon that the gas phase mobility increases with the pressure depletion rate. A possible reason is that the dispersed gas bubbles, being drawn by the free gas, gain mobility coupled with the velocity of the free gas. In such event, a more rapid pressure drop results in higher mobility of the gas bubbles by inducing faster free gas flow. The gas phase mobility consequently increases with the pressure depletion rate. The gas phase mobility, however, increases only up to a certain level. The gas relative permeability curve still remains low (Figure 5-15 and 5-16) indicating that the foamy oil flow does not diminish and maintains its effect. 
The end point of the gas relative permeability curve varies more or less proportional to the low mobility gas saturation. It appears low when the gas relative permeability evolves low and relatively high in the opposite case. Such trend is general and can be easily expected.

The residual oil saturation ranges from 10 to $50 \%$. The end point of the oil relative permeability curve lies in between 0.25 and 1 . The high residual oil saturations was also observed in the study of Ostos et al. (2004). Similarly, the low end points were necessary in the study of Ivory et al. (2009). The end point varies more or less proportional to the low mobility gas saturation. It is low when the gas relative permeability evolves low and relatively high in the opposite case.

In summary, a low pressure depletion rate affects the gas-oil flow in CSI in a way that also appears in heavy oil solution gas drive. However, a sufficiently high depletion rate yields the phenomena that do not occur in heavy oil solution gas drive. The unlike phenomena happen fundamentally because the solvent chamber accommodates the free gas independent of the pseudobubblepoint pressure of oil. The effects of the pressure depletion rate on the gas-oil flow in CSI are as below.

- At low pressure depletion rates, the critical gas saturation increases with the depletion rate to a maximum attainable value in the recovery process. The gas relative permeability evolves lower in accordance with the increasing 
critical gas saturation. Such trend of the gas relative permeability curve also appears in heavy oil solution gas drive.

- As the pressure depletion rate further increases, the critical gas saturation drops to and remains constant at the lowest value while the gas relative permeability initially appears very low but becomes higher. The critical gas saturation decreases as the free gas independent of the pseudobubblepoint pressure of oil adjoins and draws the immobile gas bubbles into the continuous gas phase. The gas relative permeability becomes higher possibly due to the phenomenon that the gas bubbles, being drawn by the free gas, gain a higher mobility with increasing velocity of the free gas.

- The end points of the gas and oil relative permeability curves vary more or less proportional to the low mobility gas saturation. The end points set low if the low mobility gas saturation is high and relatively high in the opposite case.

\subsubsection{Effect of Solvent Chamber}

The solvent chamber has significant effect on the gas-liquid relative permeability curves if the foamy oil develops a high critical gas saturation at a certain pressure depletion rate. In numerical simulations, the solvent chamber happens to insufficiently discharge the free gas when a high critical gas saturation holds an unnecessary amount of the gas phase in the porous medium. Eventually, the solvent chamber in conjunction with the high critical gas saturation causes a 
conflict in describing the simultaneous flow of free gas and foamy oil. Consequently, the gas-liquid relative permeability curves of Test 3 were misled into the shapes that counterbalance the conflict rather than representing the true characteristics of the gas-oil flow. As previously introduced, Test 3 contains the highest critical gas saturation (10\%) among the CSI tests adopted in this study

Figure 6-6 recalls the history-matching result of Test 3 , as well as the tuned gasliquid relative permeability curves in the production stages of the same test. 


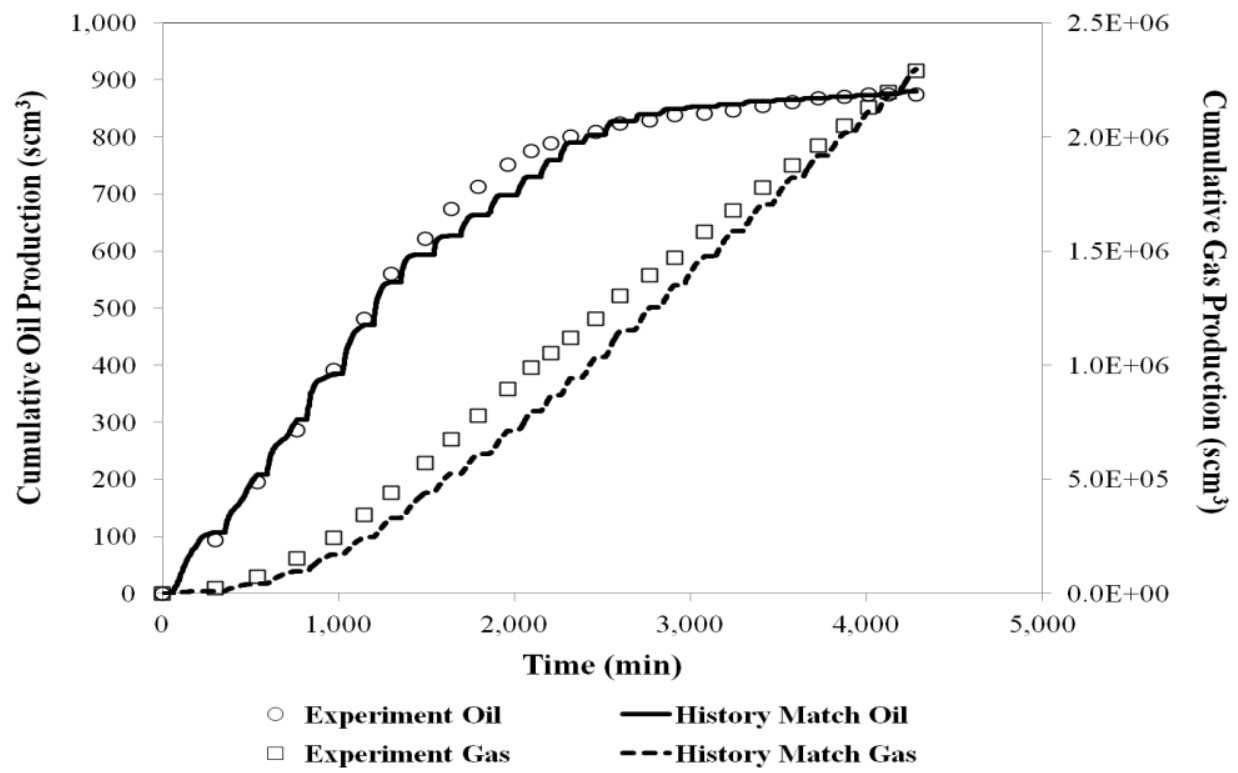

(a)

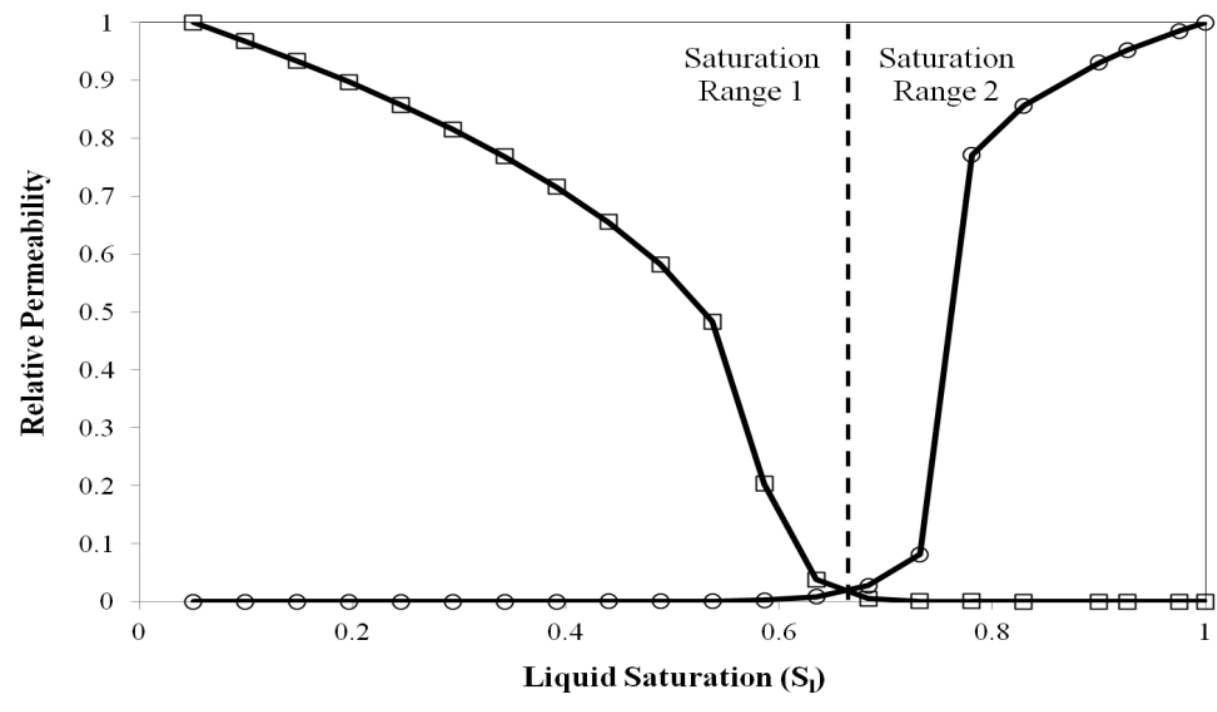

-Production/Oil (Krog_prd) -Production/Gas (Krg_prd)

(b)

Figure 6-6 Simulation results of Test 3 (a) oil and gas production profiles (b) tuned gas-liquid relative permeability curves in production stages 
The gas-liquid relative permeability curves are divided into the saturation range 1 and 2 to distinguish the differently evolving shapes.

The history-matching of Test 3 was only reasonable when the gas-liquid relative permeability curves in the production stages involved the high critical gas saturation. It was also imperative to modify the curves into the shapes as shown in the saturation range 1 and 2 .

The high critical gas saturation was essential to match the oil recovery in the early cycles, which was unexpectedly large relative to the gas production. However, the gas relative permeability curve could not describe the rising gas production after the early cycles due to the high critical gas saturation. The gasliquid fractional flow curves were therefore modified in the saturation range 1 to promote the gas production at the expense of the oil productivity. It was of necessity to increase the gas fractional flow substantially while reducing the oil flow. As a consequence, the gas relative permeability was increased along the convex curve to the maximum end point 1 . The oil relative permeability, on the other hand, was kept at a very low level. The oil relative permeability in the saturation range 2 was then modified to regain the oil recovery. It was increased steeply along the convex curve to the maximum end point 1 .

An additional simulation run was made by replacing the tuned gas-liquid relative permeability curves in the production stages of Test 3 with those of Test 4 (Figure 5-13). The additional run thereby attempted to satisfy the simultaneous 
flow of free gas and foamy oil as much as possible. As previously introduced, Test 4 resulted in the low critical gas saturation (3\%) but involved the highest low mobility gas saturation. Therefore, in the additional run, the critical gas saturation was reduced from the highest (10\%) to the low value (3\%). The gas relative permeability was decreased to the lowest level among the CSI tests adopted in this study. As a result, the low critical gas saturation eases the free gas flow in the solvent chamber. At the same time, the very low gas relative permeabilities compensate the reduced immobility of the foamy oil. Consequently, the additional run was performed in an utmost attempt to satisfy both free gas and foamy oil flows.

Figure 6-7 presents the result of the additional run in comparison to the historymatching result. 


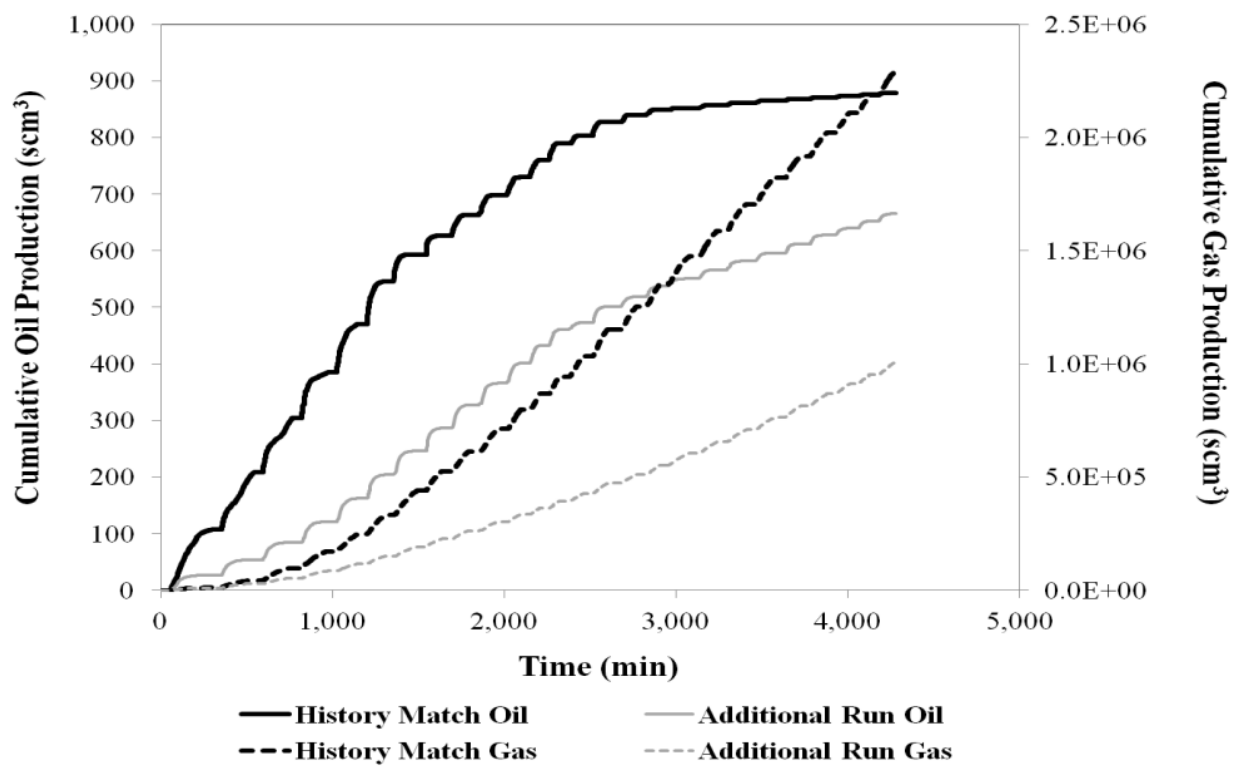

(a)

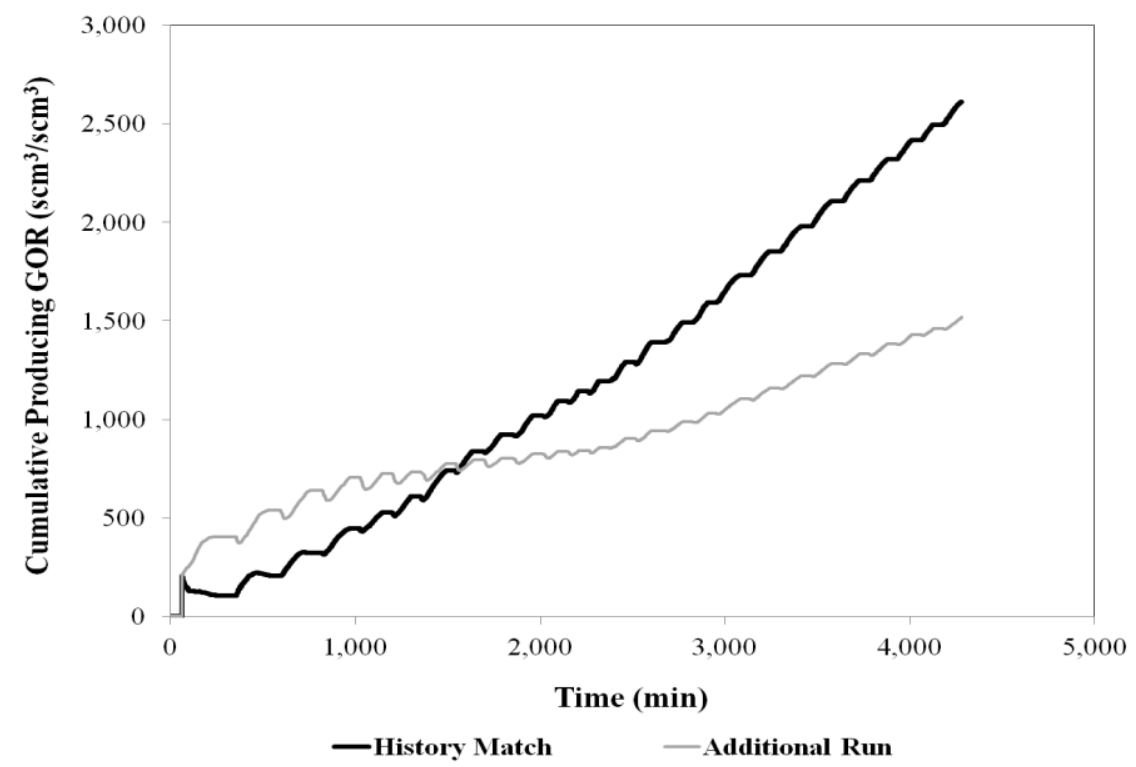

(b)

Figure 6-7 Additional run of Test 3 by replacing the $\mathrm{k}_{\text {rog_prd }}$ and $\mathrm{k}_{\mathrm{rg} \_ \text {prd }}$ curves with the ones of Test 4 (a) cumulative oil and gas productions (b) cumulative producing GOR 
As the figure shows, the additional runs yielded considerable deviations from the history-matching result despite the utmost attempt to satisfy both free gas and foamy oil flows. The unacceptable deviations, reversely, validate the tuned gasliquid relative permeability curves of Test 3 and thus the effect of the solvent chamber. It is again demonstrated that the recovery process is highly sensitive to the critical gas saturation. The decrease in the oil and gas productions as well as the higher cumulative producing GOR are opposed to what was observed in Figure 6-1 to 6-4 with the results of the increased critical gas saturation.

In summary, the effects of the solvent chamber on the gas-liquid relative permeability curves are as below.

- The solvent chamber causes the gas-liquid relative permeability curves involving a high critical gas saturation to form the shapes that inadequately reflect the simultaneous flow of foamy oil and free gas. In numerical simulations, the highly immobile gas phase insufficiently describes the free gas flow in the gas zone. As a consequence, the gas-liquid relative permeability curves were misled into the shapes that counterbalance the conflict rather than representing the true characteristics of the gas-oil flow.

It is recommended to further investigate the feasibility of incorporating a separate set of gas-liquid relative permeability curves in the solvent chamber. In this way, the simultaneous foamy oil and free gas flow can be described without 
misleading the gas-liquid relative permeability curves into the shapes that do not represent the true characteristics of the gas-oil flow.

\subsection{Gas-Liquid Relative Permeability Curves in Injection Stages}

The gas-liquid relative permeability curves in the injection stages vary from test to test (Figure 5-10 to 5-16), indicating that these fractional flow curves are also under the effect of the pressure depletion rate. The gas-liquid relative permeability curves of Test 7 were validated preliminarily through the simultaneous history-matching of Test 7, 8 and 9 . The validated curves in the injection stages were then utilized in a first attempt to history-match the rest tests. However, the reasonable history-matching results could only be attained when the curves were modified as presented in Figure 5-10 to 5-15.

The tuned gas-liquid relative permeability curves of Test 5 and 6 testify the necessity of modifying the curves in the injection stages. These curves are recalled in Figure 6-8. 


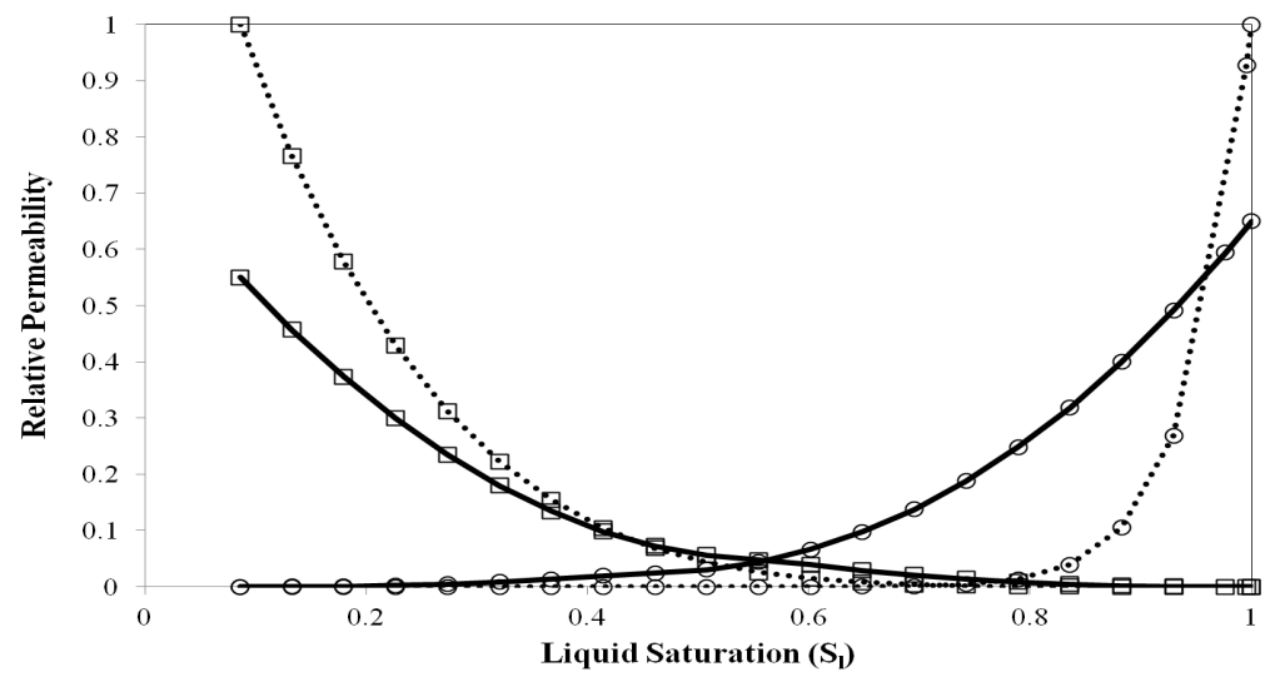

\begin{tabular}{ll}
$\cdots$ Injection/Oil (Krog_inj) & $\cdots$ \\
\hdashline Injection/Gas (Krg_inj)
\end{tabular}

(a)

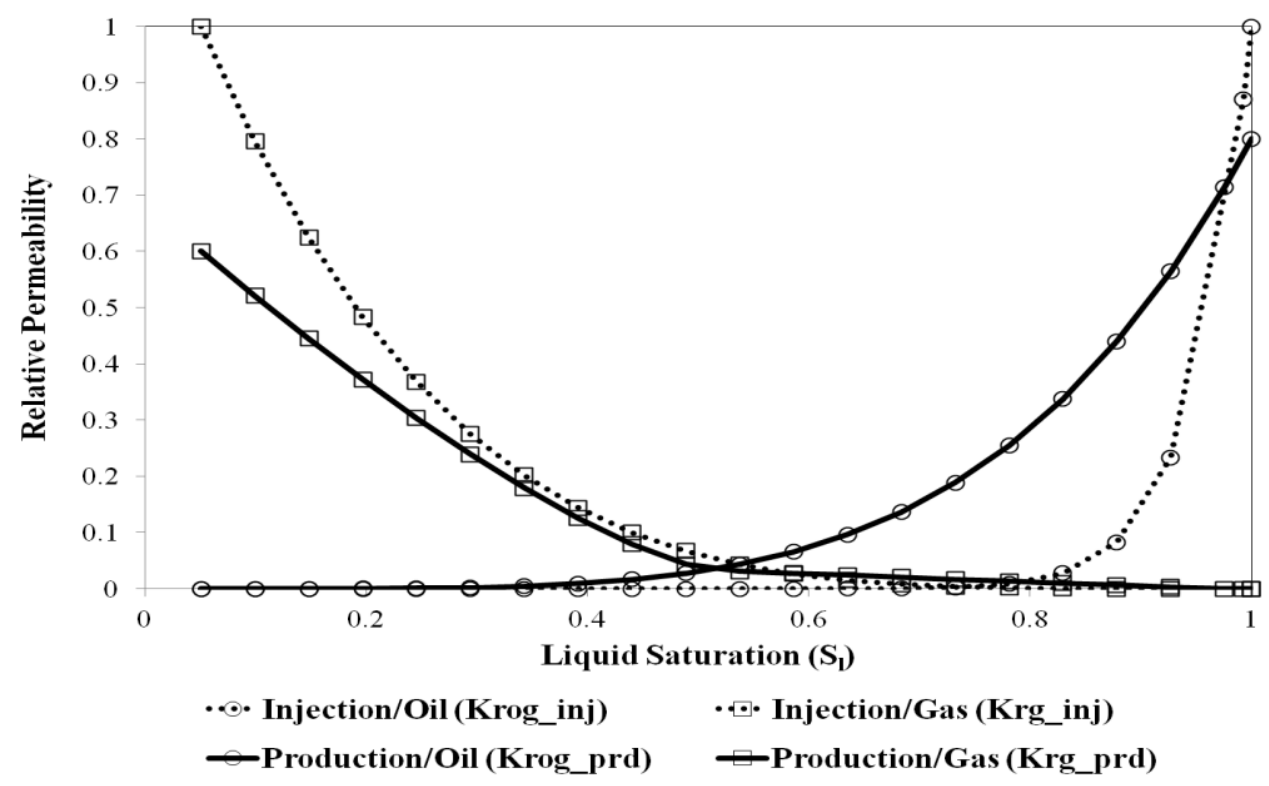

(b)

Figure 6-8 Tuned gas-liquid relative permeability curves for (a) Test $5(50 \mathrm{kPa} / 4$ min) and (b) Test $6(100 \mathrm{kPa} / 4 \mathrm{~min})$ 
As the figure shows, the gas-liquid relative permeability curves of Test 5 and 6 resulted in the resembling shapes. These curves are therefore unique as the resembling shapes history-matched the two different experimental results (Figure 5-5 and 5-6) with a similar nature of the foamy oil. The underlying assumption is that the pressure depletion rates classified next to each other (i.e. high and very high) yielded the comparable properties of the foamy oil. The unique fractional flow curves furthermore verify the necessity of modifying the gas-liquid relative permeability curves in the injection stages.

The gas-liquid relative permeability curves in the injection stages are directly related to the solvent injectivity. The curves of Test 5 were replaced by those of Test 7 to show such relationship to the solvent injectivity. The gas relative permeability of Test 7 evolves noticeably higher than that of Test 5 , representing an improved solvent injectivity. Figure 6-9 recalls the tuned gas-liquid relative permeability curves in the injection stages of Test 7.

Figure 6-10 presents the results of the additional run, such as the oil and gas production profiles as well as the cumulative producing GOR compared to the history-matching results. In addition, Figure 6-11 shows the cumulative gas injection profiles of the additional run together with the history-matching results.. 


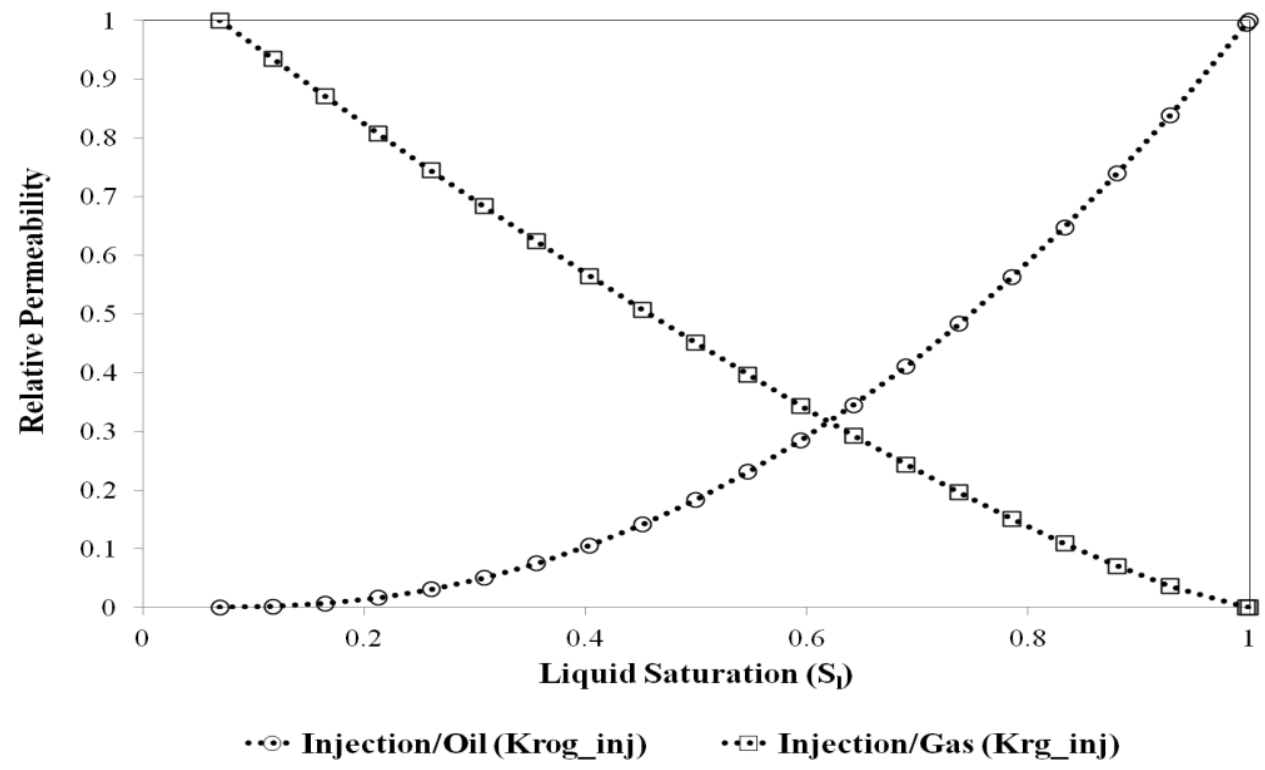

Figure 6-9 Tuned gas-liquid relative permeability curves in injection stages of Test 7 


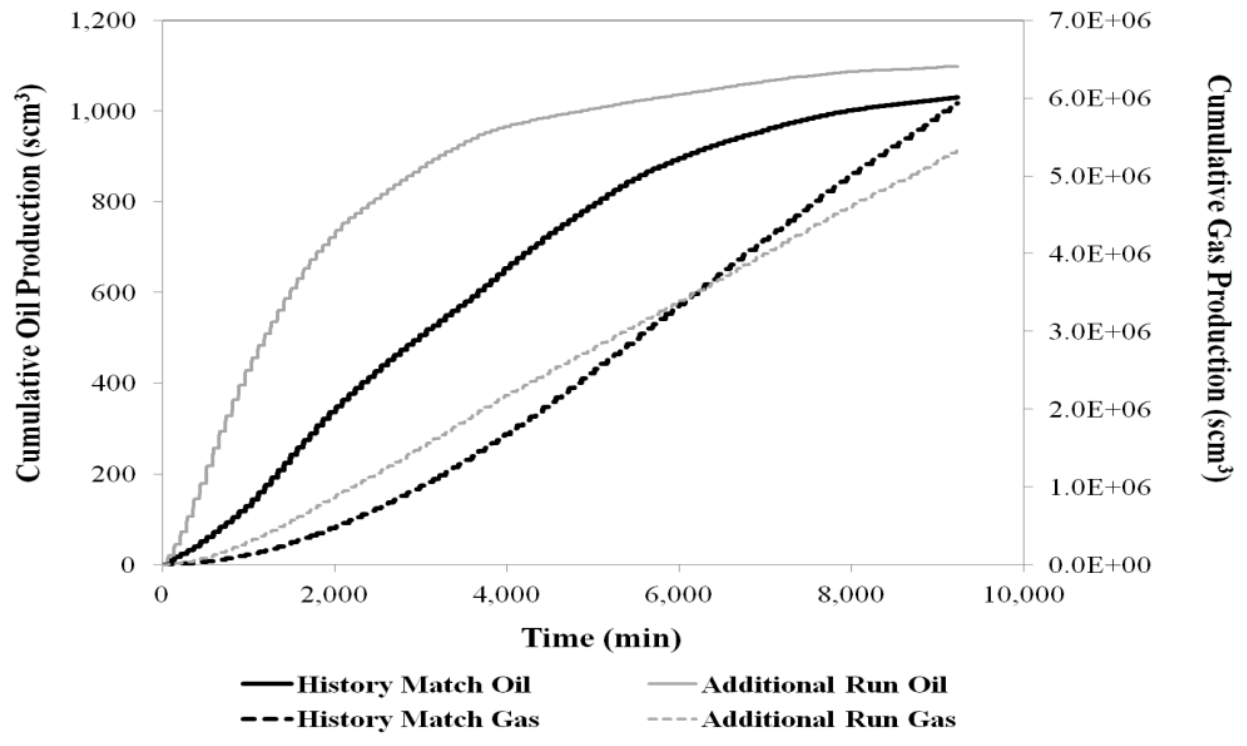

(a)

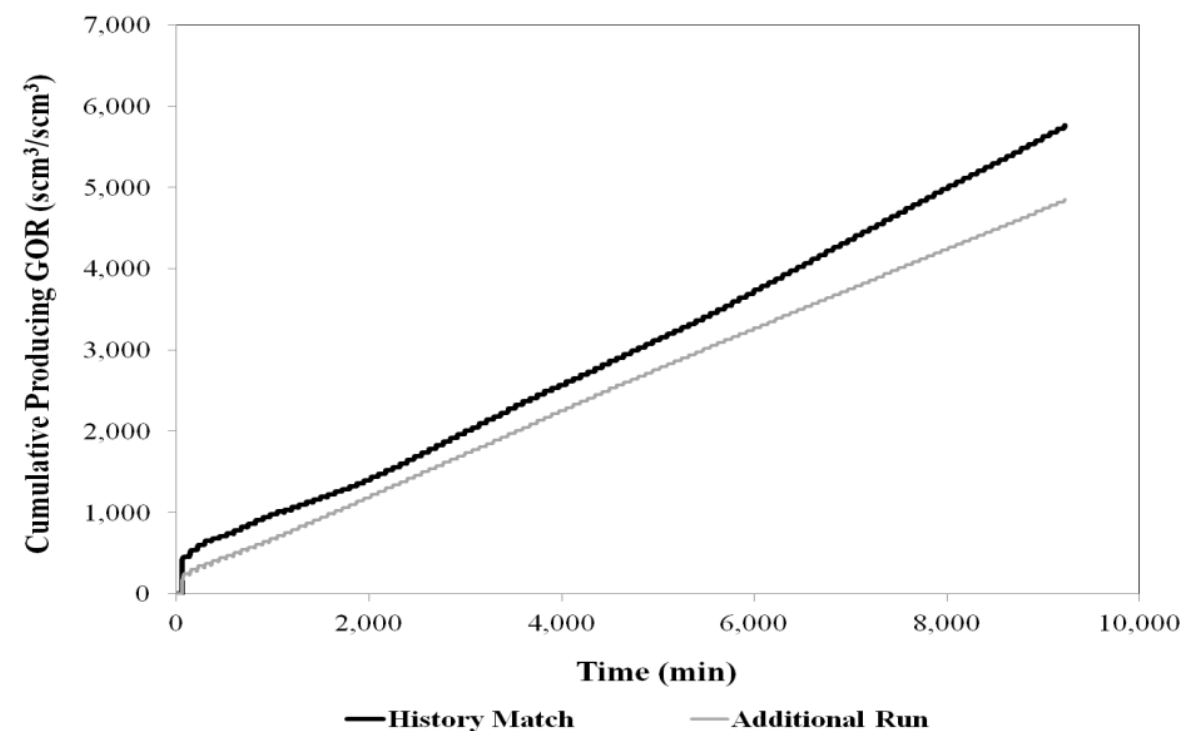

(b)

Figure 6-10 Additional run of Test 5 by replacing the $\mathrm{k}_{\text {rog_inj }}$ and $\mathrm{k}_{\mathrm{rg} \_ \text {inj }}$ curves with the ones of Test 7 (a) cumulative oil and gas productions (b) cumulative producing GOR 


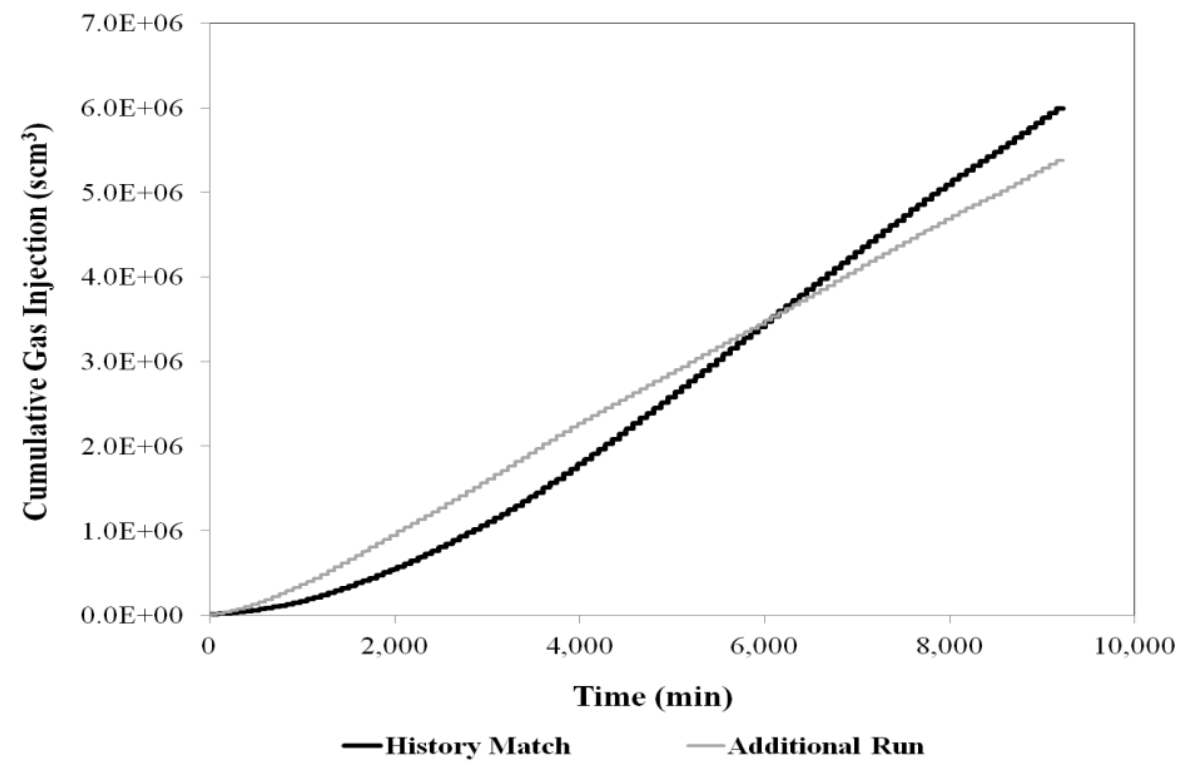

Figure 6-11 Additional run of Test 5 by replacing the krog_inj and krg_inj curves with the ones of Test 7: cumulative gas injection 
With the improved solvent injectivity, the oil production increased fairly high as expected, which ultimately increased the recovery factor by about $4 \%$. In the early cycles, the gas production increased while the cumulative producing GOR decreased. This reflects that the higher oil recovery accompanied a larger quantity of solution gas but not free gas. In the late cycles, the gas production decreased proportionally to the gas injection. The gas injection decreased since the oil phase quantifying less (due to the higher oil recovery) provided a smaller medium for the solvent to be dissolved. In this late period, the highly soluble propane more likely achieved systemwide phase equilibrium with the oil phase. The solvent injection therefore quantified proportionally to the oil phase available as the storage of the dissolved solvent.

Provided that the gas-liquid relative permeability curves in the injection stages directly impact the solvent injectivity, so far, the relationships between the factors affecting the injection stages are identified as below.

(1) The solvent injectivity is interrelated with the gas-liquid relative permeability curves in the injection stages.

(2) The gas-liquid relative permeability curves in the injection stages vary with the pressure depletion rate.

(3) The pressure depletion rate determines the nature of the foamy oil generated during the production stages. 
In the summary of these relationships, it can be postulated that the solvent injectivity is under the effect of the nature of the foamy oil present in the injection stages. The foamy oil exists in the injection stages as it has remained as unproduced in the solvent chamber during a previous production period. As a consequence, the solvent injectivity further establishes a relationship with the pressure depletion rate in the previous production stages.

Ivory et al. (2009) and Chang et al. (2013) also suggested the necessity of incorporating the kinetic foamy oil models in the injection stages. Therefore, the numerical simulation studies of CSI have consistently required accounting for the nonequilibrium phase behavior in the injection stages. However, to the author's knowledge, there has been no study investigating the solvent or gas injectivity as a function of the foamy oil existing in the injection period.

In summary, the gas-liquid relative permeability curves in the injection stages are also under the effect of the pressure depletion rate. This furthermore relates the solvent injectivity to the nature of the foamy oil present in the injection stages. Similarly, the numerical simulation studies of CSI have consistently suggested the need of accounting for the nonequilibrium phase behavior in the injection stages. However, there has been no study investigating the relationship between the solvent injectivity and the nature of the foamy oil in the injection stages. Such investigation is therefore recommended as a future study. 


\section{Chapter 7. Conclusions and Future Works}

This chapter presents the conclusions and the recommendations for the future works, which summarizes the core findings in Chapter 6.

\subsection{Conclusions}

1. The numerical simulations of CSI process necessitate incorporating two sets of gas-liquid relative permeability curves separately for the injection and production stages.

2. The modified-fractional flow model is proved to be applicable in describing the nonequilibrium phase behavior of the foamy oil in both injection and production stages of CSI process.

3. The oil and gas production profiles greatly vary with the pressure depletion rate which thereby shows the effects on the gas-oil flow in CSI process.

4. When the pressure depletion rate is low $(1 \sim 5 \mathrm{kPa} / \mathrm{min})$, the gas fractional flow curve in the production stages behaves as in heavy oil solution gas drive.

(a) The critical gas saturation increases with the pressure depletion rate until a maximum value $(10 \%)$ is attained.

(b) The gas relative permeability evolves lower and eventually results in a lower end point as the pressure depletion rate increases. 
5. In contrast, when the pressure depletion rate is sufficiently high (12.5 $\mathrm{kPa} / \mathrm{min} \sim 500 \mathrm{kPa} / 5 \mathrm{~min}$ ), the gas fractional flow curve in the production stages exhibits the behavior that does not occur in heavy oil solution gas drive.

(a) The critical gas saturation remains constant at a low value (3\%) regardless of the pressure depletion rate.

(b) The gas relative permeability curve and its end point initially appear very low but increases with the pressure depletion rate.

Such distinct behavior happens as the solvent chamber innately existing in CSI process causes the free gas to be independent of the pseudobubblepoint pressure of oil. At a sufficiently high pressure depletion rate, the free gas adjoins and draws the immobile dispersed gas bubbles into the continuous gas phase. The critical gas saturation thereby appears low and remains at the low value even if the pressure depletion rate further increases. On the other hand, the gas phase mobility (i.e. gas relative permeability) increases with the pressure depletion rate possibly in accordance with the increasing velocity of the free gas.

6. When the critical gas saturation is high, the solvent chamber in numerical simulations results in the conflict that the free gas is insufficiently discharged from the gas zone. This consequently misleads the gas-liquid relative 
permeability curves into the shapes that counterbalance the conflict rather than representing the true characteristics of the gas-oil flow.

7. The gas-liquid relative permeability curves in the injection stages are also under the effect of the pressure depletion rate. This further demonstrates that the solvent injectivity is affected by the nonequilibrium nature of the foamy oil existing in the injection stages.

\subsection{Future Works}

1. It is recommended to investigate the feasibility of incorporating another set of two-phase relative permeability curves in the solvent chamber during the production stages. In this way, the free gas discharge is possibly aided without interrupting the nonequilibrium phase behavior of the foamy oil reflected as a high critical gas saturation.

2. It is also suggested to investigate the relationship between solvent injectivity and the nonequilibrium phase behavior of the foamy oil that exists while the solvent is injected. 


\section{List of References}

Bora, R., Maini, B., \& Chakma, A. (2000). Flow Visualization Studies of Solution Gas Drive Process in Heavy Oil Reservoirs Using a Glass Micromodel. SPE Reservoir Evaluation \& Engineering , 3 (3), 224-229, SPE-64226-PA.

Bowers, B., \& Drummond, K. J. (1997). Conventional Crude Oil Resources of the Western Canada Sedimentary Basin. The Journal of Canadian Petroleum Technology, 36 (2), 56-63, PETSOC-97-02-05.

Chang, J., \& Ivory, J. (2013). Field-Scale Simulation of Cyclic Solvent Injection (CSI). Journal of Petroleum Technology, 52 (4), 251-265. SPE-157804-PA.

Das, S. K., \& Butler, R. M. (1998). Mechanism of the Vapor Extraction Process for Heavy Oil and Bitumen. Journal of Petroleum Science and Engineering , 21 (1-2), 43-59.

Dong, M., Huang, S., \& Hutchence, K. (2006). Methane Pressure-Cycling Process with Horizontal Wells for Thin Heavy-Oil Reservoirs. SPE Reservoir Evaluation \& Engineering , 9 (2), 154-164, SPE-88500-PA.

Du, Z., Chan, C., \& Zeng, F. (2013). An Experimental Study of the Post-CHOPS Cyclic Solvent Injection Process. SPE-165524-MS, presented at the SPE Heavy Oil Conference, Calgary, Alberta, Canada, 11-13 June. 
Du, Z., Zeng, F., \& Chan, C. (2014). Effects of Pressure Decline Rate on the Post-CHOPS Cyclic Solvent Injection Process. SPE-170176-MS, presented at the SPE Heavy Oil Conference, Calgary, Alberta, Canada, 10-12 June.

Ivory, J., Chang, J., Coates, R., \& Forshner, K. (2009). Investigation of Cyclic Solvent Injection Process for Heavy Oil Recovery. PETSOC-2009-161, presented at the Canadian International Petroleum Conference, Calgary, Alberta, Canada, 16-18 June.

Javadpour, F., \& Pooladi-Darvish, M. (2004). Network Modelling of ApparentRelative Permeability of Gas in Heavy Oils. Journal of Canadian Petroleum Technology , 43 (4), PETSOC-04-04-01.

Jia, X., \& Zeng, F. (2015b). Gasflooding-Assisted Cyclic Solvent Injection (GACSI) for Enhancing Heavy Oil Recovery. Fuel, 140, 344-353.

Jia, X., Li, J., \& Chen, Z. (2015a). Mathermatical Modeling of Dynamic Mass Transfer in Cyclic Solvent Injection. SPE-174519-MS, presented at the SPE Heavy Oil Conference, Calgary, AB, Canada, 09-11 June.

Kraus, W. P., McCaffrey, W. J., \& Boyd, G. W. (1993). Pseudo-Bubble Point Model For Foamy Oils. PETSOC-93-45, presented at the Annual Technical Meeting, Calgary, Alberta, Canada, 9-12 May. 
Kumar, R., \& Pooladi-Darvish, M. (2002). Solution-Gas Drive in Heavy Oil: Field Prediction and Sensitivity Studies Using Low Gas Relative Permeability. Journal of Canadian Petroleum Technology , 41 (3), PETSOC-02-03-01.

Li, K., \& Horne, R. (2004). Universal Capillary Pressure and Relative Permeability Model from Fractal Characterization of Rock. presented at the Twenty-Ninth Workshop on Geothermal Reservoir Engineering, Stanford University, Stanford, California, 26-28 January.

Li, S., \& Li, Z. (2016). Effect of Temperature on the Gas/Oil Relative Permeability of Orinoco Belt Foamy Oil. SPE Journal , 21 (1), 170-179, SPE174089-PA.

Luo, P., Yang, C., Tharanivasan, A. K., \& Gu, Y. (2007). In Situ Upgrading of Heavy Oil in a Solvent-Based Heavy Oil Recovery Process. Journal of Canadian Petroleum Technology , 46 (9), PETSOC-2005-098.

Maini, B. (2001). Foamy Oil Flow. Journal of Petroleum Technology , 53 (10), 54-64, SPE-68885-JPT.

Maini, B. (1998). Is It Futile to Measure Relative Permeability For Heavy Oil Reservoirs? Journal of Canadian Petroleum Technology , 37 (4), PETSOC-9804-06. 
Maini, B., \& Busahmin, B. (2010). Foamy Oil Flow and Its Role in Heavy Oil Production. Paper CP-1254 presented at the AIP conference proceedings, Montecatini, Italy, 20-25 June.

Ostos, A., \& Maini, B. (2004). Capillary Number in Heavy Oil Solution Gas Drive and Its Relationship with Gas-Oil Relative Permeability Curves. SPE89430-MS, presented at the SPE/DOE Symposium on Improved Oil Recovery, Tulsa, Oklahoma, 17-21 April.

Saskatchewan Energy and Mines. (2000). Annual Reservoir. Regina, SK, Canada.

Saskatchewan Petroleum Research Incentive. (2011). Husky Oil's Mervin Cold Solvent EOR Pilot. Regina, SK, Canada.

Saskatchewan Petroleum Research Incentive. (2006). Plover Lake VAPEX Huff and Puff Pilot. Regina, SK, Canada.

Talabi, O., \& Okazawa, T. (2003). Effect of Rate and Viscosity on Gas Mobility during Solution-Gas Drive in Heavy Oils. SPE-84032-MS, presented at the SPE Annual Technical Conference and Exhibition, Denver, Colorado, 5-8 October.

Tharanivasan, A. K., Yang, C., \& Gu, Y. (2004). Comparison of Three Different Interface Mass Transfer Models used in the Experimental Measurement of Solvent Diffusivity in Heavy Oil. Journal of Petroleum Science and Engineering , 44, 269-282. 
Zhang, M., Du, Z., Zeng, F., \& Xu, S. (2014). Upscaling Study of Cyclic Solvent Injection Process for Post-CHOPS Reservoirs through Numerical Simulation. SPE-170142-MS, presented at the SPE Heavy Oil Conference, Calgary, Alberta, Canada, 10-12 June.

Zhou, X., Zeng, F., Zhang, L., \& Wang, H. (2016). Foamy Oil Flow in Heavy Oil-Solvent Systems tested by Pressure Depletion in a Sandpack. Fuel, 171, 210-223. 


\section{Appendix I: Grid Block Coordinates}

$* * \$ * * * * * * * * * * * * * * * * * * * * * * * * * * * * * * * * * * * * * * * * * * * * * * * * * * * * * * * * * * * * * *$
$* * \$$ Definition of fundamental corner point grid
$* * \$ * * * * * * * * * * * * * * * * * * * * * * * * * * * * * * * * * * * * * * * * * * * * * * * * * * * * * * * * *$

GRID CORNER 121010

CORNERS

$* * \$ \mathrm{X}$-Coordinates

**\$ Base Coordinates Start

$0.00002 * 2.54002 * 5.08002 * 7.62002 * 10.1600$

$2 * 12.70002 * 15.24002 * 17.78002 * 20.32002 * 22.8600$

$2 * 25.40002 * 27.940030 .4800$

$* * \$$ Base Coordinates End

**\$ The Base Coordinates are repeated 399 times to complete the X-Coordinates

**\$ Y-Coordinates

$168 * 4.572048 * 6.096048 * 7.620048 * 9.1440168 * 10.6680$

$120 * 3.048048 * 4.572048 * 6.096048 * 7.620048 * 9.1440$

$48 * 10.6680120 * 12.1920$

$120 * 3.048048 * 4.572048 * 6.096048 * 7.620048 * 9.1440$

$48 * 10.6680120 * 12.192072 * 1.524048 * 3.048048 * 4.5720$

$48 * 6.096048 * 7.620048 * 9.144048 * 10.668048 * 12.1920$

$72 * 13.7160$

$72 * 1.524048 * 3.048048 * 4.572048 * 6.096048 * 7.6200$

$48 * 9.144048 * 10.668048 * 12.192072 * 13.716024 * 0.6362$

$48 * 1.524048 * 3.048048 * 4.572048 * 6.096048 * 7.6200$

$48 * 9.144048 * 10.668048 * 12.192048 * 13.716024 * 14.6038$

$24 * 0.636248 * 1.524048 * 3.048048 * 4.572048 * 6.0960$

$48 * 7.620048 * 9.144048 * 10.668048 * 12.192048 * 13.7160$

$24 * 14.603824 * 0.154048 * 1.524048 * 3.048048 * 4.5720$

$48 * 6.096048 * 7.620048 * 9.144048 * 10.668048 * 12.1920$

$$
\text { A-1 }
$$




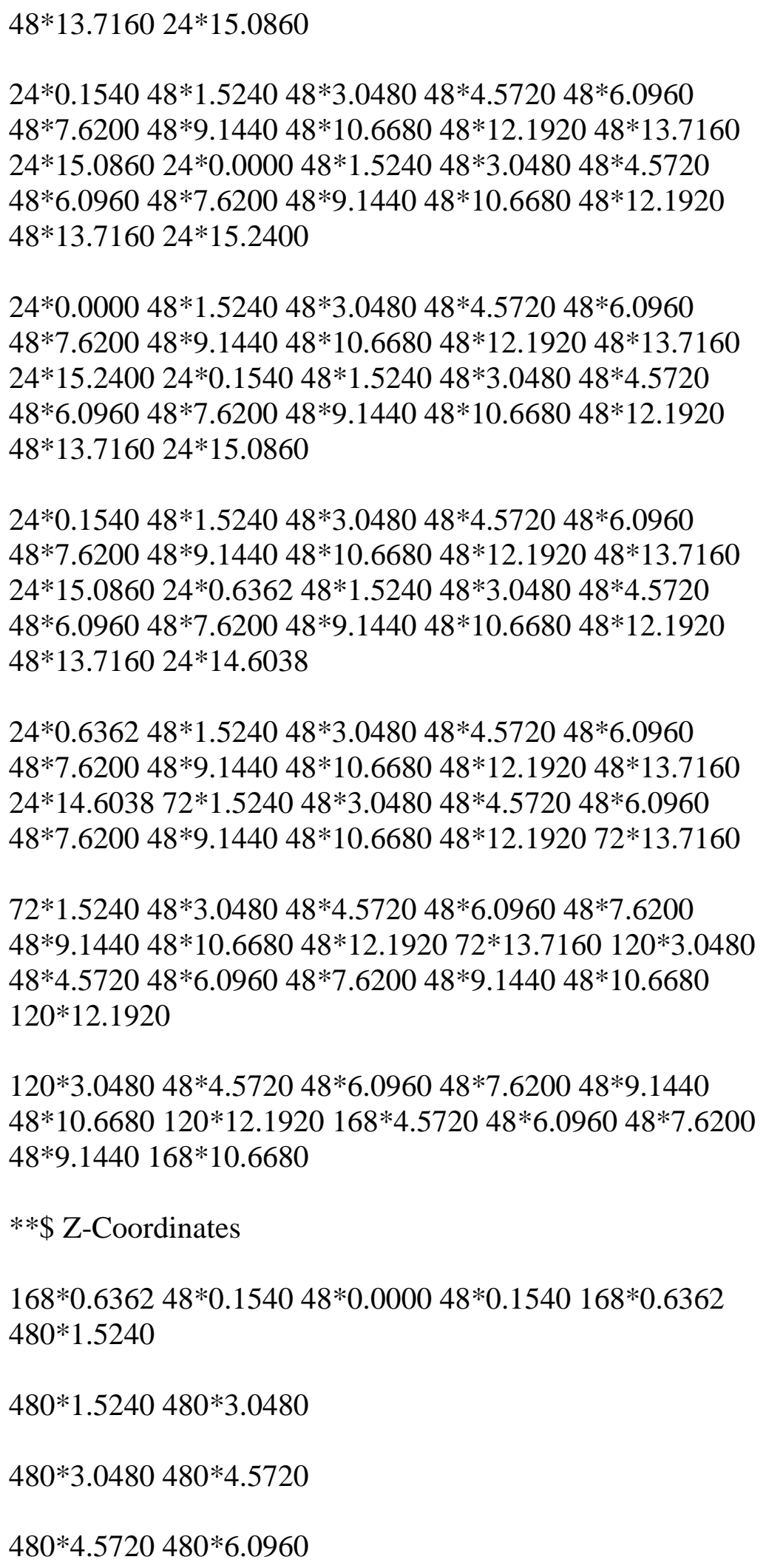


$480 * 6.0960480 * 7.6200$

$480 * 7.6200480 * 9.1440$

$480 * 9.1440480 * 10.6680$

$480 * 10.6680480 * 12.1920$

$480 * 12.1920480 * 13.7160$

$480 * 13.7160168 * 14.603848 * 15.086048 * 15.240048 * 15.0860$

$168 * 14.6038$

**\$ Refining grid blocks for the tests with the injector and producer (mimic

**\$ wormhole) at center - Test 1 to 7

REFINE 1,6,6 INTO 122

CORNERS RG 1,6,6

**\$ X-Coordinates

0.00002 .54000 .00002 .54000 .00002 .54000 .00002 .5400

0.00002 .54000 .00002 .54000 .00002 .54000 .00002 .5400

0.00002 .54000 .00002 .54000 .00002 .54000 .00002 .5400

0.00002 .54000 .00002 .54000 .00002 .54000 .00002 .5400

$* * \$$ Y-Coordinates

$2 * 7.62004 * 7.81002 * 9.14402 * 7.62004 * 7.81002 * 9.1440$

$2 * 7.62004 * 7.81002 * 9.14402 * 7.62004 * 7.81002 * 9.1440$

**\$ Z-Coordinates

$8 * 7.620016 * 7.81008 * 9.1440$

REFINE 2,6,6 INTO 122

CORNERS RG 2,6,6

$* * \$ \mathrm{X}$-Coordinates

2.54005 .08002 .54005 .08002 .54005 .08002 .54005 .0800

2.54005 .08002 .54005 .08002 .54005 .08002 .54005 .0800

2.54005 .08002 .54005 .08002 .54005 .08002 .54005 .0800 
2.54005 .08002 .54005 .08002 .54005 .08002 .54005 .0800

**\$Y-Coordinates

$2 * 7.62004 * 7.81002 * 9.14402 * 7.62004 * 7.81002 * 9.1440$

$2 * 7.62004 * 7.81002 * 9.14402 * 7.62004 * 7.81002 * 9.1440$

**\$ Z-Coordinates

$8 * 7.620016 * 7.81008 * 9.1440$

REFINE 3,6,6 INTO 122

CORNERS RG 3,6,6

$* * \$ \mathrm{X}$-Coordinates

5.08007 .62005 .08007 .62005 .08007 .62005 .08007 .6200

5.08007 .62005 .08007 .62005 .08007 .62005 .08007 .6200

5.08007 .62005 .08007 .62005 .08007 .62005 .08007 .6200

5.08007 .62005 .08007 .62005 .08007 .62005 .08007 .6200

**\$ Y-Coordinates

$2 * 7.62004 * 7.81002 * 9.14402 * 7.62004 * 7.81002 * 9.1440$

$2 * 7.62004 * 7.81002 * 9.14402 * 7.62004 * 7.81002 * 9.1440$

**\$ Z-Coordinates

$8 * 7.620016 * 7.81008 * 9.1440$

REFINE 4,6,6 INTO 122

CORNERS RG 4,6,6

$* * \$ \mathrm{X}$-Coordinates

7.620010 .16007 .620010 .16007 .620010 .16007 .620010 .1600

7.620010 .16007 .620010 .16007 .620010 .16007 .620010 .1600

7.620010 .16007 .620010 .16007 .620010 .16007 .620010 .1600

7.620010 .16007 .620010 .16007 .620010 .16007 .620010 .1600

$* * \$ Y$-Coordinates

$2 * 7.62004 * 7.81002 * 9.14402 * 7.62004 * 7.81002 * 9.1440$

$2 * 7.62004 * 7.81002 * 9.14402 * 7.62004 * 7.81002 * 9.1440$ 
$* * \$$ Z-Coordinates

$8 * 7.620016 * 7.81008 * 9.1440$

**\$ Refining grid blocks for the test with the injector and producer (mimic

**\$ wormhole) at bottom - Test 8

REFINE 1,6,9 INTO 122

CORNERS RG $1,6,9$

**\$ X-Coordinates

0.00002 .54000 .00002 .54000 .00002 .54000 .00002 .5400

0.00002 .54000 .00002 .54000 .00002 .54000 .00002 .5400

0.00002 .54000 .00002 .54000 .00002 .54000 .00002 .5400

0.00002 .54000 .00002 .54000 .00002 .54000 .00002 .5400

**\$ Y-Coordinates

$2 * 7.62004 * 7.81002 * 9.14402 * 7.62004 * 7.81002 * 9.1440$

$2 * 7.62004 * 7.81002 * 9.14402 * 7.62004 * 7.81002 * 9.1440$

**\$ Z-Coordinates

$8 * 12.192016 * 12.38208 * 13.7160$

REFINE 2,6,9 INTO 122

CORNERS RG 2,6,9

**\$ X-Coordinates

2.54005 .08002 .54005 .08002 .54005 .08002 .54005 .0800

2.54005 .08002 .54005 .08002 .54005 .08002 .54005 .0800

2.54005 .08002 .54005 .08002 .54005 .08002 .54005 .0800

2.54005 .08002 .54005 .08002 .54005 .08002 .54005 .0800

**\$ Y-Coordinates

$2 * 7.62004 * 7.81002 * 9.14402 * 7.62004 * 7.81002 * 9.1440$

$2 * 7.62004 * 7.81002 * 9.14402 * 7.62004 * 7.81002 * 9.1440$

**\$ Z-Coordinates 
$8 * 12.192016 * 12.38208 * 13.7160$

REFINE 3,6,9 INTO 122

CORNERS RG 3,6,9

$* * \$ \mathrm{X}$-Coordinates

5.08007 .62005 .08007 .62005 .08007 .62005 .08007 .6200

5.08007 .62005 .08007 .62005 .08007 .62005 .08007 .6200

5.08007 .62005 .08007 .62005 .08007 .62005 .08007 .6200

5.08007 .62005 .08007 .62005 .08007 .62005 .08007 .6200

**\$ Y-Coordinates

$2 * 7.62004 * 7.81002 * 9.14402 * 7.62004 * 7.81002 * 9.1440$

$2 * 7.62004 * 7.81002 * 9.14402 * 7.62004 * 7.81002 * 9.1440$

**\$ Z-Coordinates

$8 * 12.192016 * 12.38208 * 13.7160$

REFINE 4,6,9 INTO 122

CORNERS RG 4,6,9

**\$ X-Coordinates

7.620010 .16007 .620010 .16007 .620010 .16007 .620010 .1600

7.620010 .16007 .620010 .16007 .620010 .16007 .620010 .1600

7.620010 .16007 .620010 .16007 .620010 .16007 .620010 .1600

7.620010 .16007 .620010 .16007 .620010 .16007 .620010 .1600

$* * \$ Y$-Coordinates

$2 * 7.62004 * 7.81002 * 9.14402 * 7.62004 * 7.81002 * 9.1440$

$2 * 7.62004 * 7.81002 * 9.14402 * 7.62004 * 7.81002 * 9.1440$

**\$ Z-Coordinates

$8 * 12.192016 * 12.38208 * 13.7160$

**\$ Refining grid blocks for the test with the injector and producer (mimic $* * \$$ wormhole) at top - Test 9

REFINE 1,6,2 INTO 122 
CORNERS RG 1,6,2

$* * \$ \mathrm{X}$-Coordinates

0.00002 .54000 .00002 .54000 .00002 .54000 .00002 .5400

0.00002 .54000 .00002 .54000 .00002 .54000 .00002 .5400

0.00002 .54000 .00002 .54000 .00002 .54000 .00002 .5400

0.00002 .54000 .00002 .54000 .00002 .54000 .00002 .5400

**\$ Y-Coordinates

$2 * 7.62004 * 7.81002 * 9.14402 * 7.62004 * 7.81002 * 9.1440$

$2 * 7.62004 * 7.81002 * 9.14402 * 7.62004 * 7.81002 * 9.1440$

**\$ Z-Coordinates

$8 * 1.524016 * 2.85808 * 3.0480$

REFINE 2,6,2 INTO 122

CORNERS RG 2,6,2

$* * \$ \mathrm{X}$-Coordinates

2.54005 .08002 .54005 .08002 .54005 .08002 .54005 .0800

2.54005 .08002 .54005 .08002 .54005 .08002 .54005 .0800

2.54005 .08002 .54005 .08002 .54005 .08002 .54005 .0800

2.54005 .08002 .54005 .08002 .54005 .08002 .54005 .0800

**\$ Y-Coordinates

$2 * 7.62004 * 7.81002 * 9.14402 * 7.62004 * 7.81002 * 9.1440$

$2 * 7.62004 * 7.81002 * 9.14402 * 7.62004 * 7.81002 * 9.1440$

**\$ Z-Coordinates

$8 * 1.524016 * 2.85808 * 3.0480$

REFINE 3,6,2 INTO 122

CORNERS RG 3,6,2

$* * \$ \mathrm{X}$-Coordinates

5.08007 .62005 .08007 .62005 .08007 .62005 .08007 .6200

5.08007 .62005 .08007 .62005 .08007 .62005 .08007 .6200 
5.08007 .62005 .08007 .62005 .08007 .62005 .08007 .6200

5.08007 .62005 .08007 .62005 .08007 .62005 .08007 .6200

**\$ Y-Coordinates

$2 * 7.62004 * 7.81002 * 9.14402 * 7.62004 * 7.81002 * 9.1440$

$2 * 7.62004 * 7.81002 * 9.14402 * 7.62004 * 7.81002 * 9.1440$

**\$ Z-Coordinates

$8 * 1.524016 * 2.85808 * 3.0480$

REFINE 4,6,2 INTO 122

CORNERS RG 4,6,2

$* * \$ \mathrm{X}$-Coordinates

7.620010 .16007 .620010 .16007 .620010 .16007 .620010 .1600

7.620010 .16007 .620010 .16007 .620010 .16007 .620010 .1600

7.620010 .16007 .620010 .16007 .620010 .16007 .620010 .1600

7.620010 .16007 .620010 .16007 .620010 .16007 .620010 .1600

**\$ Y-Coordinates

$2 * 7.62004 * 7.81002 * 9.14402 * 7.62004 * 7.81002 * 9.1440$

$2 * 7.62004 * 7.81002 * 9.14402 * 7.62004 * 7.81002 * 9.1440$

**\$ Z-Coordinates

$8 * 1.524016 * 2.85808 * 3.0480$

**\$ Refining grid blocks for observer (model pressure)

REFINE 12,6,6 INTO 122

CORNERS RG 12,6,6

$* * \$ \mathrm{X}$-Coordinates

27.940030 .480027 .940030 .480027 .940030 .480027 .940030 .4800

27.940030 .480027 .940030 .480027 .940030 .480027 .940030 .4800

27.940030 .480027 .940030 .480027 .940030 .480027 .940030 .4800

27.940030 .480027 .940030 .480027 .940030 .480027 .940030 .4800

**\$ Y-Coordinates 
$2 * 7.62004 * 7.81002 * 9.14402 * 7.62004 * 7.81002 * 9.1440$
$2 * 7.62004 * 7.81002 * 9.14402 * 7.62004 * 7.81002 * 9.1440$
$* * \$$ Z-Coordinates
$8 * 7.620016 * 7.81008 * 9.1440$
$* * \$ 0=$ pinched block, $1=$ active block
PINCHOUTARRAY CON 1
*MOD
1:12 $1: 11: 1=0$
$1: 121: 12: 2=0$
$1: 121: 19: 9=0$
$1: 121: 110: 10=0$
$1: 12$ 2:2 $1: 1=0$
$1: 12$ 2:2 $10: 10=0$
$1: 12$ 9:9 $1: 1=0$
$1: 12$ 9:9 $10: 10=0$
$1: 1210: 101: 1=0$
$1: 1210: 102: 2=0$
$1: 1210: 109: 9=0$
$1: 1210: 1010: 10=0$
END-GRID 


\section{Appendix II: PVT Model}

$* * * * * * * * * * * * * * * * * * * * * * * * * * * * * * * * * * * * * * * * * * * * * * * * * * * * * * * * * * * * * * * * * * * *$

THE FOLLOWING SECTION CAN BE USED FOR THE COMPONENT

PROPERTY INPUT INTO STARS

$* * * * * * * * * * * * * * * * * * * * * * * * * * * * * * * * * * * * * * * * * * * * * * * * * * * * * * * * * * * * * * * * * * * *$

** PVT UNITS CONSISTENT WITH *INUNIT *SI

MODEL 3331

COMPNAME 'WATER' 'C3H8' 'Oil'

CMM

00.04410 .389

PCRIT

04245.52606 .98

TCRIT

0.0096 .65501 .85

KV1

000

KV2

000

KV3

000

KV4

000

KV5

000

**\$ Gas-liquid K Value tables

KVTABLIM 1050101070

KVTABLE 'C3H8'

66.794

0.83438

0.83438

0.83438

0.83438

0.83438 


$\begin{array}{llllll}92.865 & 1.0804 & 1.0804 & 1.0804 & 1.0804 & 1.0804 \\ 123.97 & 1.399 & 1.399 & 1.399 & 1.399 & 1.399 \\ 159.84 & 1.8115 & 1.8115 & 1.8115 & 1.8115 & 1.8115 \\ 199.98 & 2.2504 & 1.2617 & 1.2617 & 1.2617 & 1.2617\end{array}$

KVTABLE 'Oil'

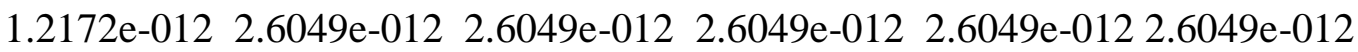

$2.6798 \mathrm{e}-011 \quad 1.8013 \mathrm{e}-011 \quad 1.8013 \mathrm{e}-011 \quad 1.8013 \mathrm{e}-011 \quad 1.8013 \mathrm{e}-0111.8013 \mathrm{e}-011$

$\begin{array}{llllll}4.1424 \mathrm{e}-010 & 1.2456 \mathrm{e}-010 & 1.2456 \mathrm{e}-010 & 1.2456 \mathrm{e}-010 & 1.2456 \mathrm{e}-010 & 1.2456 \mathrm{e}-010\end{array}$

4.7359e-009 8.6131e-010 8.6131e-010 8.6131e-010 8.6131e-010 8.6131e-010

4.1787e-008 5.1862e-009 $6.183 \mathrm{e}-008 \quad 6.183 \mathrm{e}-008 \quad 6.183 \mathrm{e}-008 \quad 6.183 \mathrm{e}-008$

** reference pressure, corresponding to the density

PRSR 101.325

** reference temperature, corresponding to the density

TEMR 22

** pressure at surface, for reporting well rates, etc.

PSURF 101.325

** temperature at surface, for reporting well rates, etc.

TSURF 22

$* * \$$ Surface conditions

SURFLASH KVALUE

MOLDEN

00.012940 .002483

$\mathrm{CP}$

00 1.171e-006

CT1

$00-0.0001359$

CT2

00 2.228e-006

CPT

$002.642 \mathrm{e}-015$ 


\section{VISCTABLE}

*ATPRES 101.325

**\$ temp

$\begin{array}{llll}15 & 0 & 2.139370111 & 4376.2 \\ 20 & 0 & 2.004734123 & 2760.4 \\ 25 & 0 & 1.878571119 & 1789.3 \\ 30 & 0 & 1.760347874 & 1189.5 \\ 35 & 0 & 1.649564717 & 809.53 \\ 40 & 0 & 1.545753425 & 563.08\end{array}$

*ATPRES 2000

**\$ temp

$\begin{array}{lcll}15 & 0 & 0.15901 & 4870 \\ 20 & 0 & 0.15305 & 3075.6 \\ 25 & 0 & 0.14751 & 1995.8 \\ 30 & 0 & 0.14235 & 1328.1 \\ 35 & 0 & 0.13752 & 904.66 \\ 40 & 0 & 0.133 & 629.74\end{array}$




\title{
Appendix III: Gas-Liquid Relative Permeability Tables
}

\author{
**\$ Test 1 \\ ROCKFLUID \\ $* * \$$ Injection Stages \\ RPT 1 WATWET
}

** Sl $\quad \mathrm{krg} \quad \mathrm{krog}$

SLT

$0.03380000000000000000 \quad 1.00000000000000000000$

$0.05857435897435900000 \quad 0.96668715071122100000$

$0.08334871794871800000 \quad 0.93363950043187600000$

$0.10812307692307700000 \quad 0.90086202537129200000$

$0.13289743589743600000 \quad 0.86835993550549300000$

$\begin{array}{lll}0.15767179487179500000 & 0.83613869253183000000\end{array}$

$0.18244615384615400000 \quad 0.80420402976977300000$

$0.20722051282051300000 \quad 0.77256197428405000000$

$0.23199487179487200000 \quad 0.74121887155532400000$

$0.25676923076923100000 \quad 0.71018141308315800000$

0.71018141308315800000

0.28154358974359000000

0.64905211489590900000

0.30631794871794900000

0.61897568755474600000

0.33109230769230800000

0.58923581366105200000

0.35586666666666700000

0.58923581366105200000

0.38064102564102600000

0.55984146918576300000

0.40541538461538500000

0.53080223659939000000

0.43018974358974400000

0.50212837273312100000

0.45496410256410300000

0.47383088750324300000

0.47973846153846200000

0.44592163580929600000

0.50451282051282100000

0.41841342554089500000

0.52928717948718000000

0.39132014546112400000

0.55406153846153800000

0.36465691786003100000

0.57883589743589700000

0.33844028241480500000

0.60361025641025600000

0.31268841984210500000

0.00000000000000000000 0.00000000106266313917 0.00000005299132660158 0.00000052162631551468 0.00000264249374189885 0.00000930221645186780 0.00002601169592754230 0.00006205096816218770 0.00013177200164237500 0.00025604916836651500 0.00046386928458435600 0.00079405459027277200 0.00129711309591297000 0.00203721152012495000 0.00309426665763955000 0.00456615150659332000 0.00657101288132507000 0.00924969756475810000 0.01276828432913380000 0.01732071938685680000 0.02313155303299760000 0.03045877541394520000 0.03959674950767200000 0.05087923953384170000 0.62838461538461500000 0.28742142697349100000 0.06468253312954630000 0.65315897435897400000 0.26266165928579600000 0.08142865573115100000 0.67793333333333300000 0.23843416341793500000 0.10158867569649200000 0.70270769230769200000 0.21476723204418400000 0.12568609878605600000 0.72748205128205100000 0.19169312878906900000 0.15430035069806200000 0.75225641025641000000 0.16924905548828400000 0.18807034642162100000 0.77703076923076900000 0.14747847518147600000 0.22769814523526000000 0.80180512820512800000 0.82657948717948700000 0.12643297585435100000 0.27395269023583100000 0.85135384615384600000 0.10617499158904800000 0.32767363133576800000 0.87612820512820500000 0.08678195573191240000 0.06835300838859610000 0.38977523071540000000 0.46125034976201500000 
$0.90090256410256400000 \quad 0.05102067090498990000$ $0.92567692307692300000 \quad 0.03497340168889220000$ 0.95045128205128200000 0.99940000000000000000 1.00000000000000000000

**\$ Production Stages

\section{RPT 2 WATWET}

** S1

SLT

0.03380000000000000000 0.05857435897435900000 0.08334871794871800000 0.10812307692307700000 0.13289743589743600000 0.15767179487179500000 0.18244615384615400000 0.20722051282051300000 0.23199487179487200000 0.25676923076923100000 0.28154358974359000000 0.30631794871794900000 0.33109230769230800000 0.355866666666666700000 0.38064102564102600000 0.40541538461538500000 0.43018974358974400000 0.45496410256410300000 0.47973846153846200000 0.50451282051282100000 0.52928717948718000000 0.55406153846153800000 0.57883589743589700000 0.60361025641025600000 0.62838461538461500000 0.65315897435897400000 0.67793333333333300000 0.70270769230769200000 0.72748205128205100000 0.75225641025641000000 0.77703076923076900000 0.80180512820512800000 0.82657948717948700000 0.85135384615384600000 0.87612820512820500000
0.02050664379942510000 0.00000000000000000000 0.00000000000000000000 $\mathrm{krg}$

0.50000000000000000000 0.46770067019113400000 0.43668340316215500000 0.40693100178558600000 0.37842602202820100000 0.35115076214860900000 0.32508725108557700000 0.30021723594967800000 0.27652216851833900000 0.25398319061955700000 0.23258111827204100000 0.21229642442854500000 0.19310922014406700000 0.17499923396016700000 0.15794578925972200000 0.14192777930117200000 0.12692363958538900000 0.11291131713867900000 0.09986823620794700000 0.08777125975294880000 0.07659664597802330000 0.06631999896057720000 0.05691621218995620000 0.04835940350505290000 0.04062283947752670000 0.03367884667739270000 0.02749870639702790000 0.02205252816671630000 0.01730909555109620000 0.01323567489412560000 0.00979777319999897000 0.00695882390437765000 0.00467976627837231000 0.00291845984815771000 0.00162882547133842000
0.54317451656899000000 0.63671007310690000000 0.74311040121446200000 0.99650266160392800000 1.00000000000000000000 krog

0.00000000000000000000 0.00000000000000000003 0.00000000000000014615 0.00000000000001998851 0.00000000000065506948 0.00000000000981309100 0.00000000008959305394 0.00000000058121779001 0.00000000293617012595 0.00000001225353266692 0.00000004398450141589 0.00000013976323499504 0.00000040157640542901 0.00000106030921762879 0.00000260515007146141 0.00000601570658903684 0.00001316058101617370 0.00002745649460824860 0.00005492311496949390 0.00010582319095837400 0.00019714851984386800 0.00035630319648587000 0.00062645054555300200 0.00107413365523359000 0.00179995660712300000 0.00295333001095176000 0.00475254660399060000 0.00751176741753454000 0.01167687399030450000 0.01787258568837200000 0.02696376249964850000 0.04013442262839860000 0.05898871157117140000 0.08567887672422630000 0.12306624146844000000

$$
\text { A-14 }
$$


$0.90090256410256400000 \quad 0.00075949043465506500$ $0.92567692307692300000 \quad 0.00025140305671381600$ 0.97000000000000000000 0.97522564102564100000 0.00000000000000000000 0.00000000000000000000 1.00000000000000000000

$* * \$$ Test 2

ROCKFLUID

**\$ Injection Stages

RPT 1 WATWET

** S1

SLT

0.09510000000000000000 0.11830256410256400000 0.14150512820512800000 0.16470769230769200000 0.18791025641025600000 0.21111282051282100000 0.23431538461538500000 0.25751794871794900000 0.28072051282051300000 0.30392307692307700000 0.32712564102564100000 0.35032820512820500000 0.37353076923076900000 0.39673333333333300000 0.41993589743589700000 0.44313846153846200000 0.46634102564102600000 0.48954358974359000000 0.51274615384615400000 0.53594871794871800000 0.55915128205128200000 0.58235384615384600000 0.60555641025641000000 0.62875897435897400000 0.65196153846153800000 0.67516410256410300000 0.69836666666666700000 0.72156923076923100000 0.74477179487179500000 0.76797435897435900000 0.79117692307692300000 $\mathrm{krg}$

1.00000000000000000000 0.89202363433252500000 0.79328003900026200000 0.70319805230111200000 0.62122761484632600000 0.54683954476057400000 0.47952530902980700000 0.41879679081758800000 0.36418605255661100000 0.31524509460664600000 0.27154560925290100000 0.23267872979945400000 0.19825477449080800000 0.16790298497016300000 0.14127125895546400000 0.11802587678289600000 0.09785122143174670000 0.08044949160350410000 0.06554040738073790000 0.05286090793637650000 0.04216484069988680000 0.03322264131144290000 0.02582100360583040000 0.01976253876105550000 0.01486542261784840000 0.01096303001929370000 0.00790355482630617000 0.00554961402294168000 0.00377783401907165000 0.00247841686278146000 0.00155468355520059000
0.17492224855139400000 0.24617786886036100000 0.44335520229435400000 0.47432356377829700000 0.65000000000000000000 krog

0.00000000000000000000 0.00000938071284308044 0.00008384759844418120 0.00030195276214008800 0.00074945474639942400 0.00151698419315142000 0.00269894349955601000 0.00439284265976294000 0.00669884918975403000 0.00971946077970855000 0.01355925542133560000 0.01832469381897640000 0.02412395886750040000 0.03106682242810000000 0.03926453282662470000 0.04882971847739740000 0.05987630431678150000 0.07251943859174380000 0.08687542814532600000 0.10306168076548200000 0.12119665347275400000 0.14139980585171200000 0.16379155770443100000 0.18849325043734700000 0.21562711169633200000 0.24531622284640600000 0.27768448895751800000 0.31285661101012000000 0.35095806007681800000 0.39211505327121700000 0.43645453128386900000 
$0.81437948717948700000 \quad 0.00092259096124753900$ $0.83758205128205100000 \quad 0.00051021784289324400$ $\begin{array}{lll}0.86078461538461500000 & 0.00025721415971694000\end{array}$ $0.88398717948718000000 \quad 0.00011420572448978800$ 0.90718974358974400000 0.93039230769230800000 0.95359487179487200000 0.99820000000000000000 1.00000000000000000000

$* * \$$ Production Stages

\section{RPT 2 WATWET}

** $\mathrm{Sl}$

SLT

0.09510000000000000000 0.11830256410256400000 0.14150512820512800000 0.16470769230769200000 0.18791025641025600000 0.21111282051282100000 0.23431538461538500000 0.25751794871794900000 0.28072051282051300000 0.30392307692307700000 0.32712564102564100000 0.35032820512820500000 0.37353076923076900000 0.39673333333333300000 0.41993589743589700000 0.44313846153846200000 0.46634102564102600000 0.48954358974359000000 0.51274615384615400000 0.53594871794871800000 0.55915128205128200000 0.58235384615384600000 0.60555641025641000000 0.62875897435897400000 0.65196153846153800000 0.67516410256410300000 0.698366666666666700000 0.72156923076923100000 0.74477179487179500000 0.76797435897435900000 0.79117692307692300000
0.00004214307644788230 0.00001157802027636840 0.00000184127598611169 0.00000000000000000000 0.00000000000000000000 $\mathrm{krg}$

0.40000000000000000000 0.33929441839002500000 0.28643109136902100000 0.24058383942935300000 0.20099270063150400000 0.16696039052401300000 0.13784885109449700000 0.11307588919166100000 0.09211190487444500000 0.07447671016190970000 0.05973643867615310000 0.04750054669070340000 0.03741890611854780000 0.02917898999747050000 0.02250315105588590000 0.01714599397013180000 0.01289184195453670000 0.00955229835885906000 0.00696390398436010000 0.00498589087032051000 0.00349803334790674000 0.00239859720869951000 0.00160238789190049000 0.00103889865844848000 0.00065055979356347600 0.00039108996360390400 0.00022395095121570600 0.00012090710811665900 0.00006069100236215680 0.00002777690343809950 0.00001126395400400130
0.48410413734922000000 0.53519219750750500000 0.58984770204247400000 0.64820028799018800000 0.71038022262638000000 0.77651838785032100000 0.84674626539223200000 0.99372771593929000000 1.00000000000000000000 krog

0.00000000000000000000 0.00000000012272690382 0.00000000807534251030 0.00000009348720082187 0.00000053135176254870 0.00000204511909688226 0.00000615139096194186 0.00001560744954293180 0.00003496256600924110 0.00007121386138846510 0.00013456737412243300 0.00023930490272550700 0.00040475712647293300 0.00065638345540561600 0.00102695901913121000 0.00155786916933980000 0.00230051184188648000 0.00331780809949834000 0.00468582115475517000 0.00649548415431708000 0.00885443698893271000 0.01188897237917670000 0.01574609147383540000 0.02059566918614780000 0.02663272948252170000 0.03407983082872940000 0.04318956198981110000 0.05424714837187520000 0.06757316908658870000 0.08352638491232830000 0.10250667731964500000 
$\begin{array}{lll}0.81437948717948700000 & 0.00000387113666715678\end{array}$

$\begin{array}{lll}0.83758205128205100000 & 0.00000104648179043953\end{array}$

0.86078461538461500000

0.88398717948718000000

0.93000000000000000000

0.93039230769230800000

0.95359487179487200000

0.97679743589743600000

1.00000000000000000000

**\$ Test 3

\section{ROCKFLUID}

**\$ Injection Stages

RPT 1 WATWET

** $\mathrm{Sl}$

SLT

0.05050000000000000000

0.07484615384615390000

0.09919230769230770000

0.12353846153846200000

0.14788461538461500000

0.17223076923076900000

0.19657692307692300000

0.22092307692307700000

0.24526923076923100000

0.26961538461538500000

0.29396153846153900000

0.31830769230769200000

0.34265384615384600000

0.36700000000000000000

0.39134615384615400000

0.41569230769230800000

0.44003846153846200000

0.46438461538461500000

0.48873076923076900000

0.51307692307692300000

0.53742307692307700000

0.56176923076923100000

0.58611538461538500000

0.61046153846153900000

0.63480769230769200000

0.65915384615384600000

0.68350000000000000000
0.00000019341855573392

0.00000001782080244227

0.00000000000000000000

0.00000000000000000000

0.00000000000000000000

0.00000000000000000000

0.00000000000000000000 $\mathrm{krg}$

1.00000000000000000000 0.90696371687868700000 0.82039627430019100000 0.74000813040974100000 0.66551513411870500000 0.59663857368058100000 0.53310522712550400000 0.47464741468200900000 0.42100305332795900000 0.37191571362751700000 0.32713467902814200000 0.28641500781116400000 0.24951759791208900000 0.21620925485289000000 0.18626276305893900000 0.15945696086878500000 0.13557681958680300000 0.11441352697828900000 0.09576457566562450000 0.07943385695513920000 0.06523176071026350000 0.05297528199165140000 0.04248813531471090000 0.03360087753687460000 0.02615104059146940000 0.01998327554691070000 0.01494950981036520000
0.12495809872282700000

0.15137203411388800000

0.18229047423021000000

0.21830940040228700000

0.30745007002286200000

0.30832368118024300000

0.36381298137456700000

0.42739821247224200000

0.50000000000000000000 krog

0.00000000000000000000 0.00070744401711047100 0.00279081770509002000 0.00622862468251563000 0.01100958277215550000 0.01712587172093390000 0.02457149346364590000 0.03334158160217330000 0.04343204237090580000 0.05483934346367480000 0.06756037913657210000 0.08159237873772460000 0.09693284180836960000 0.11357949036427500000 0.13153023278239000000 0.15078313581094100000 0.17133640243651400000 0.19318835408081400000 0.21633741606728100000 0.24078210560336600000 0.26652102172983300000 0.29355283683030500000 0.32187628939421000000 0.35149017779819000000 0.38239335492358100000 0.41458472346665700000 0.44806323182774600000 
0.70784615384615400000 0.73219230769230800000 0.75653846153846200000 0.78088461538461500000 0.80523076923076900000 0.82957692307692300000 0.85392307692307700000 0.87826923076923100000 0.90261538461538500000 0.92696153846153900000 0.95130769230769200000 0.98020000000000000000 1.00000000000000000000

**\$ Production Stages

\section{RPT 2 WATWET}

** S1

SLT

0.05050000000000000000 0.07484615384615390000 0.09919230769230770000 0.12353846153846200000 0.14788461538461500000 0.17223076923076900000 0.19657692307692300000 0.22092307692307700000 0.24526923076923100000 0.26961538461538500000 0.29396153846153900000 0.31830769230769200000 0.34265384615384600000

0.36700000000000000000 0.39134615384615400000 0.41569230769230800000 0.44003846153846200000 0.46438461538461500000 0.48873076923076900000 0.51307692307692300000 0.53742307692307700000 0.56176923076923100000 0.58611538461538500000 0.61046153846153900000 0.63480769230769200000 0.65915384615384600000 0.68350000000000000000
0.01090911974526070000 0.00772912157932792000 0.00528438431787252000 0.00345786956447008000 0.00214090488779790000 0.00123350001092276000 0.00064471929729647800 0.00029313109627828000 0.00010736744846871800 0.00002685395215362090 0.00000283254614190235 0.00000000000000000000 0.00000000000000000000
0.48282787048781900000 0.51887766879846900000 0.55621169212478300000 0.59482903929125500000 0.63472884028939400000 0.67591025421250400000 0.71837246738860400000 0.76211469168696600000 0.80713616297741100000 0.85343613972455800000 0.90101390170171500000 0.95913285185050400000 1.00000000000000000000 $\mathrm{krg}$

1.00000000000000000000 0.98400695647671500000 0.96757835156564000000 0.95067961095635600000 0.93327141891736200000 0.91530876303581500000 0.89673971762045600000 0.87750387300425000000 0.85753027646846800000 0.83673468600407100000 0.81501583506359300000 0.79225023648008900000 0.76828476297742500000 0.74292572279769700000 0.71592217542376500000 0.68693929304859500000 0.65551341656881500000 0.62097068589614400000 0.58226524477433500000 0.53761222496881500000 0.48346855229859000000 0.41043989305346200000 0.20347085419005600000 0.09097382330781500000 0.03789722481508000000 0.01450941731729860000 0.00501441507260926000 krog

0.00000000000000000000 0.00000000000000000000 0.00000000000000000087 0.00000000000000035085 0.00000000000002485813 0.00000000000067718259 0.00000000001007813189 0.00000000009882390070 0.00000000071404030489 0.00000000408593622977 0.00000001945181002527 0.00000007979683875416 0.00000028949047134103 0.00000094724226386396 0.00000283867862648980 0.00000788626862384667 0.00002051053376374860 0.00005033945913489430 0.00011736695032873600 0.00026139715080793400 0.00055874577885103800 0.00115087398839456000 0.00229213357325792000 0.00442740520022321000 0.00831550270485378000 0.01522129306725520000 0.02720917054998110000

$$
\text { A-18 }
$$


$0.70784615384615400000 \quad 0.00152646325772181000$ $0.73219230769230800000 \quad 0.00039543882277271400$ $0.75653846153846200000 \quad 0.00008286245271682830$ $0.78088461538461500000 \quad 0.00001297491580843930$ $0.80523076923076900000 \quad 0.00000132763454709288$ 0.82957692307692300000 0.85392307692307700000 0.90000000000000000000 0.90261538461538500000 0.92696153846153900000 0.95130769230769200000 0.97565384615384600000 1.00000000000000000000

**\$ Test 4

\section{ROCKFLUID}

**\$ Injection Stages

\section{RPT 1 WATWET}

** Sl

SLT

0.04170000000000000000 0.06627179487179490000 0.09084358974358970000 0.11541538461538500000 0.13998717948717900000 0.16455897435897400000 0.18913076923076900000 0.21370256410256400000 0.23827435897435900000 0.26284615384615400000 0.28741794871794900000 0.31198974358974400000 0.33656153846153900000 0.36113333333333300000 0.38570512820512800000 0.41027692307692300000 0.43484871794871800000 0.45942051282051300000 0.48399230769230800000 0.50856410256410300000 0.53313589743589700000 0.55770769230769200000 0.58227948717948700000
0.00000006877387956274 0.00000000100146368847 0.00000000000000000000 0.00000000000000000000 0.00000000000000000000 0.00000000000000000000 0.00000000000000000000 0.00000000000000000000 $\mathrm{krg}$

1.00000000000000000000 0.88721134281644800000 0.78460627258921500000 0.69150652878644300000 0.60726208973652400000 0.53125074130856800000 0.46287764015913800000 0.40157487131639500000 0.34680099985598600000 0.29804061640445700000 0.25480387618524800000 0.21662603129941200000 0.18306695590743800000 0.15371066394975200000 0.12816481901099900000 0.10606023589644200000 0.08705037344711800000 0.07081081807267090000 0.05703875742608340000 0.04545244358131070000 0.03579064500137410000 0.02781208649852500000 0.02129487628675940000
0.04758360772863060000 0.08153995427704030000 0.69545220167989700000 0.77127030470408900000 0.81989061581701500000 0.85645193609981200000 0.88605331009380100000 0.93063125236534900000 0.93284021864415200000 0.95215349071298400000 0.96955237250588900000 0.98541063152483400000 1.00000000000000000000 krog

0.00000000000000000000 0.00000000000689734233 0.00000000089208698082 0.00000001533512723176 0.00000011538055426080 0.00000055202196937205 0.00000198341139115454 0.00000584850051396154 0.00001492306533750680 0.00003409517983133890 0.00007139729887289060 0.00013933219152764100 0.00025653003637381600 0.00044977405333022900 0.00075643210292621100 0.00122733173531188000 0.00193011621840643000 0.00295311911808091000 0.00440979504368675000 0.00644374420998466000 0.00923436850193408000 0.01300319676214740000 0.01802091705232330000 
$0.60685128205128200000 \quad 0.01603591910648940000$ $0.63142307692307700000 \quad 0.01185031425662470000$ $0.65599487179487200000 \quad 0.00857073719407112000$ 0.68056666666666700000 0.70513846153846100000 0.72971025641025600000 0.75428205128205100000 0.77885384615384600000 0.80342564102564100000 0.82799743589743600000 0.85256923076923100000 0.87714102564102600000 0.90171282051282100000 0.92628461538461500000 0.95085641025641000000 0.98930000000000000000 1.00000000000000000000

**\$ Production Stages

\section{RPT 2 WATWET}

** Sl

SLT

0.04170000000000000000 0.06627179487179490000 0.09084358974358970000 0.11541538461538500000 0.13998717948717900000 0.16455897435897400000 0.18913076923076900000 0.21370256410256400000 0.23827435897435900000 0.26284615384615400000 0.28741794871794900000 0.31198974358974400000 0.33656153846153900000 0.36113333333333300000 0.38570512820512800000 0.41027692307692300000 0.43484871794871800000 0.45942051282051300000 0.48399230769230800000 0.50856410256410300000 0.53313589743589700000 0.55770769230769200000 0.58227948717948700000
0.00604680314574263000

0.00414441091117978000 0.00274506469719460000 0.00174517139416893000 0.00105531013879969000 0.00059947025186652500 0.00031425259867295200 0.00014802793780630600 0.00006004362321537970 0.00001946657527061050 0.00000434462314417628 0.00000045745239650917 0.00000000000000000000 0.00000000000000000000 $\mathrm{krg}$

0.35000000000000000000 0.30910151007329200000 0.27056526200538300000 0.23442079310249200000 0.20070042693945500000 0.16943980517466400000 0.14067858238757400000 0.11446135693108000000 0.09083895634809950000 0.06987028022816240000 0.05162507122267140000 0.03618835179150710000 0.02366817411848940000 0.01421104144581730000 0.00804030392054296000 0.00563130596517032000 0.00535035934958602000 0.00507121350541407000 0.00479394276796943000 0.00451862844695623000 0.00424535988661858000 0.00397423575572451000 0.00370536563389267000
0.02461515366984440000 0.03317902672911670000 0.04418053214428570000 0.05817277987580730000 0.07580512832808040000 0.09783525280906420000 0.12514218598558200000 0.15874036828992000000 0.19979474625445200000 0.24963695677137200000 0.30978263529430800000 0.38194988601760800000 0.46807895208750600000 0.57035312391723900000 0.69122092369551900000 0.92425498317553300000 1.00000000000000000000 $\operatorname{krog}$

0.00000000000000000000 0.00000000250531706632 0.00000008171297076635 0.00000062746543209183 0.00000266513555558108 0.00000818341156733637 0.00002046529966151440 0.00004442148706598570 0.00008692558186303640 0.00015715091465366900 0.00026690867960760100 0.00043098724897502700 0.00066749253236039300 0.00099818927792218500 0.00144884323138688000 0.00204956408276866000 0.00283514914144019000 0.00384542768864081000 0.00512560596325845000 0.00672661274220548000 0.00870544548122500000 0.01112551698572960000 0.01405700258444650000 
$0.60685128205128200000 \quad 0.00343887198546490000$ $0.63142307692307700000 \quad 0.00317489264829012000$ $\begin{array}{lll}0.65599487179487200000 & 0.00291358401896659000\end{array}$ $\begin{array}{lll}0.68056666666666700000 & 0.00265512519939801000\end{array}$ 0.70513846153846100000 0.72971025641025600000 0.75428205128205100000 0.77885384615384600000 0.80342564102564100000 0.82799743589743600000 0.85256923076923100000 0.87714102564102600000 0.90171282051282100000 0.92628461538461500000 0.97000000000000000000 0.97542820512820500000 1.00000000000000000000

$* * \$$ Test 5

\section{ROCKFLUID}

**\$ Injection Stages

\section{RPT 1 WATWET}

** S1

SLT

0.08610000000000000000 0.10953333333333300000 0.132966666666666700000 0.15640000000000000000 0.17983333333333300000 0.203266666666666700000 0.22670000000000000000 0.25013333333333300000 0.273566666666666700000 0.29700000000000000000 0.32043333333333300000 0.34386666666666700000 0.36730000000000000000 0.39073333333333300000 0.41416666666666700000 0.43760000000000000000 0.46103333333333300000 0.48446666666666700000 0.50790000000000000000
0.00239972350144329000 0.00214762192256418000 0.00189910957371386000 0.00165453669846564000 0.00141433716467214000 0.00117906382319393000 0.00094944767277734400 0.00072650546883524800 0.00051175998079861600 0.00030778168152852500 0.00000000000000000000 0.00000000000000000000 0.00000000000000000000 $\operatorname{krg} \quad \operatorname{krog}$

1.00000000000000000000 0.87663959540654800000 0.76579035207881200000 0.66647219186694800000 0.57775797874380000000 0.49877201721223200000 0.42868855259836000000 0.36673027328242400000 0.31216681492201800000 0.26431326672566900000 0.22252867983830600000 0.18621457790408800000 0.15481346987632800000 0.12780736514902000000 0.10471629108968400000 0.08509681305907860000 0.06854055700981570000 0.04315067207152380000 0.05467273476316880000
0.01757718778134060000 0.02177081636360070000 0.02673043894547430000 0.03255676192947410000 0.03935899686800680000 0.04725521020981120000 0.05637267341678530000 0.06684821343783450000 0.07882856352732000000 0.09247071439653040000 0.10794226568736600000 0.12542177775811200000 0.14509912377179900000 0.16717584207822700000 0.21305633838743700000 0.21939399118332700000 0.25000000000000000000
0.00000000000000000000 0.00000000000000000000 0.00000000000000000000 0.00000000000000000054 0.00000000000000006030 0.00000000000000234233 0.00000000000004658230 0.00000000000058364437 0.00000000000521454274 0.00000000003598467624 0.00000000020255382314 0.00000000096689759804 0.00000000402821720402 0.00000001496964228651 0.00000005047080478884 0.00000015647216558834 0.00000045092899367840 0.00000121871668059840 0.00000311178460918869

$$
\text { A-21 }
$$




$\begin{array}{llll}0.53133333333333300000 & 0.03366233958315290000 & 0.00000755265245244438 \\ 0.55476666666666700000 & 0.02592488683645510000 & 0.00001751589663461210 \\ 0.57820000000000000000 & 0.01968317942278140000 & 0.00003898853155148260 \\ 0.60163333333333300000 & 0.01470833947071090000 & 0.00008361273127834190 \\ 0.62506666666666700000 & 0.01079628962057470000 & 0.00017333128450536200 \\ 0.64850000000000000000 & 0.00776630067659539000 & 0.00034834117211059700 \\ 0.67193333333333300000 & 0.00545954314587237000 & 0.00068038790855224500 \\ 0.69536666666666700000 & 0.00373764289940358000 & 0.00129450386512230000 \\ 0.71880000000000000000 & 0.00248124122151290000 & 0.00240384342838150000 \\ 0.74223333333333300000 & 0.00158855955194723000 & 0.00436447649345992000 \\ 0.76566666666666700000 & 0.00097396927161904700 & 0.00776010522661595000 \\ 0.78910000000000000000 & 0.00056656694148873200 & 0.01353097283564870000 \\ 0.81253333333333300000 & 0.00030875547879379500 & 0.02316712935849510000 \\ 0.83596666666666700000 & 0.00015483185239350600 & 0.03899420667776260000 \\ 0.85940000000000000000 & 0.00006958200991086680 & 0.06459056131936850000 \\ 0.88283333333333300000 & 0.00002688393102884040 & 0.10538885454498800000 \\ 0.90626666666666700000 & 0.00000831996441962455 & 0.16953382948394000000 \\ 0.92970000000000000000 & 0.00000180000894543161 & 0.26909241563190400000 \\ 0.95313333333333300000 & 0.00000019776784674829 & 0.42174381229604200000 \\ 0.99579999000000000000 & 0.00000000000000000000 & 0.92723967656681900000 \\ 1.00000000000000000000 & 0.00000000000000000000 & 1.00000000000000000000\end{array}$

**\$ Production Stages

RPT 2 WATWET

** S1

SLT

0.08610000000000000000
0.10953333333333300000
0.13296666666666700000
0.15640000000000000000
0.17983333333333300000
0.20326666666666700000
0.22670000000000000000
0.25013333333333300000
0.27356666666666700000
0.29700000000000000000
0.32043333333333300000
0.34386666666666700000
0.36730000000000000000
0.39073333333333300000
0.41416666666666700000
0.43760000000000000000
0.46103333333333300000
0.48446666666666700000
0.50790000000000000000 krg

0.55

0.5024233682

0.4571723884

0.4142531072

0.3736719343

0.3354356878

0.2995516486

0.2660276258

0.2348720374

0.2060940109

0.1797035103

0.1557115008

0.1341301668

0.1149732095

0.09825626695

0.0839975372

0.07221875638

0.0629468713

0.05621727662 krog

0

2.1989297e-005

0.0001314827364

0.0003742692806

0.000786187479

0.00139815061

0.002237904611

0.003330920662

0.004700927049

0.006370257965

0.008360097553

0.0106906589

0.01338132011

0.01645073074

0.01963434022

0.02066772655

0.02440305719

0.0265501048

0.03047094741

$$
\text { A-22 }
$$




$\begin{array}{lll}0.53133333333333300000 & 0.05208157122 & 0.03630654049 \\ 0.55476666666666700000 & 0.04753577107 & 0.04416223672 \\ 0.57820000000000000000 & 0.04236883943 & 0.05412330825 \\ 0.60163333333333300000 & 0.03914434969 & 0.06626191954 \\ 0.62506666666666700000 & 0.03398576037 & 0.08064092634 \\ 0.64850000000000000000 & 0.02921497282 & 0.0973161948 \\ 0.67193333333333300000 & 0.02482791322 & 0.1163381296 \\ 0.69536666666666700000 & 0.02082024659 & 0.1377527383 \\ 0.71880000000000000000 & 0.01718733733 & 0.1616024046 \\ 0.74223333333333300000 & 0.01392419967 & 0.1879264687 \\ 0.76566666666666700000 & 0.01102543436 & 0.2167616753 \\ 0.78910000000000000000 & 0.008485145703 & 0.2481425264 \\ 0.81253333333333300000 & 0.006296829718 & 0.2821015654 \\ 0.83596666666666700000 & 0.004453217889 & 0.3186696087 \\ 0.85940000000000000000 & 0.00294604861 & 0.3578759372 \\ 0.88283333333333300000 & 0.001765711836 & 0.3997484571 \\ 0.90626666666666700000 & 0.0009006470832 & 0.4443138356 \\ 0.92970000000000000000 & 0.0003361802801 & 0.4915976174 \\ 0.97000000000000000000 & 0 & 0.5793430945 \\ 0.97656666666666700000 & 0 & 0.5944175432 \\ 1.00000000000000000000 & 0 & 0.65\end{array}$

**\$ Test 6

ROCKFLUID

$* * \$$ Injection Stages

RPT 1 WATWET
** $\mathrm{S} 1$
$\mathrm{krg}$
$\operatorname{krog}$
SLT
0.051000000000000000001 .00000000000000000000
0.00000000000000000000
$0.07533333333333330000 \quad 0.89307626633253000000$
0.00000000000000000000
$\begin{array}{lll}0.09966666666666670000 & 0.79516432044521800000\end{array}$
0.00000000000000000000
$0.12400000000000000000 \quad 0.70571941414086300000$
0.00000000000000000001
$0.14833333333333300000 \quad 0.62421604210641600000$
0.00000000000000000110
$\begin{array}{lll}0.17266666666666700000 & 0.55014777253900200000\end{array}$
0.00000000000000006303
$0.19700000000000000000 \quad 0.48302707451320200000$
0.00000000000000172780
0.22133333333333300000
0.42238514193013000000
0.00000000000002839534
0.24566666666666700000
0.36777171387600000000
0.00000000000032090826
0.27000000000000000000
0.31875489120359900000
0.00000000000272464991
0.29433333333333300000
0.27492094913411400000
0.00000000001846148945
0.31866666666666700000
0.23587414565883300000
0.00000000010422163905
0.34300000000000000000
0.20123652550012300000
0.00000000050605551786
0.36733333333333300000
0.17064771936825200000
0.00000000216510027057
0.39166666666666700000
0.14376473822482600000
0.00000000831671586418 
$0.41600000000000000000 \quad 0.12026176223409800000$ $\begin{array}{lll}0.44033333333333300000 & 0.09982992404969210000\end{array}$ $0.46466666666666700000 \quad 0.08217708604535290000$ $0.48900000000000000000 \quad 0.06702761105338140000$ $\begin{array}{lll}0.51333333333333300000 & 0.05412212612190260000\end{array}$ $\begin{array}{lll}0.53766666666666700000 & 0.04321727874061940000\end{array}$ 0.56200000000000000000 0.58633333333333300000 0.61066666666666700000 0.63500000000000000000 0.65933333333333300000 0.683666666666666700000 0.70800000000000000000 0.73233333333333300000 0.75666666666666700000 0.78100000000000000000 0.80533333333333300000 0.829666666666666700000 0.85400000000000000000 0.87833333333333300000 0.90266666666666700000 0.92700000000000000000 0.95133333333333300000 0.99280000000000000000 1.00000000000000000000

**\$ Production Stages

\section{RPT 2 WATWET}

** S1

SLT

$0.05100000000000000000 \quad 0.60000000000000000000$ $0.07533333333333330000 \quad 0.55990320073073200000$ $\begin{array}{lll}0.09966666666666670000 & 0.52059196544371700000\end{array}$ $\begin{array}{lll}0.12400000000000000000 & 0.48209577649266600000\end{array}$ 0.14833333333333300000 0.17266666666666700000 0.19700000000000000000 0.22133333333333300000 0.24566666666666700000 0.27000000000000000000 0.29433333333333300000 0.31866666666666700000 0.34300000000000000000 0.36733333333333300000 0.39166666666666700000
0.03408548491199050000

0.02651466835819240000 0.02030799005018370000 0.01528356711897240000 0.01127418005437270000 0.00812696690435355000 0.00570310294620576000 0.00387746399158157000 0.00253827108514086000 0.00158671382207388000 0.00093654878228348000 0.00051366855967884300 0.00025563538561679400 0.00011117110233355100 0.00003959164481150180 0.00001016797187872030 0.00000138343480860079 0.00000000000000000000 0.00000000000000000000
0.44444711789681500000

0.40768199973596900000

0.37184061787313600000

0.33696819695845900000

0.30311608705552700000

0.27034322005272900000

0.23871809149348500000

0.20832153657268700000

0.17925075734822400000

0.15162542453668600000

0.12559744855054400000
0.00000002911280160816 0.00000009399085958706 0.00000028263057926893 0.00000079802303793545 0.00000213025763096651 0.00000540718785104295 0.00001311502518067230 0.00003052548831966120 0.00006842963736020280 0.00014821866111186900 0.00031106940169417500 0.00063413647702643400 0.00125844052005572000 0.00243588390349820000 0.00460695932897700000 0.00852685194265770000 0.01546660863822270000 0.02752899409850370000 0.04813709576020610000 0.08277970414287680000 0.14013364605254200000 0.23373306296286400000 0.38442360357300300000 0.87083389000551600000 1.00000000000000000000 $\operatorname{krog}$

0.00000000000000000000 0.00000009248010205940 0.00000189906172787672 0.00001112492190350240 0.00003899688004204060 0.00010317097131145400 0.00022844820606911600 0.00044737916522370200 0.00080079369232174300 0.00133827585181007000 0.00211859674332543000 0.00321011364500493000 0.00469114150273424000 0.00665030121462954000 0.00918684810685076000 


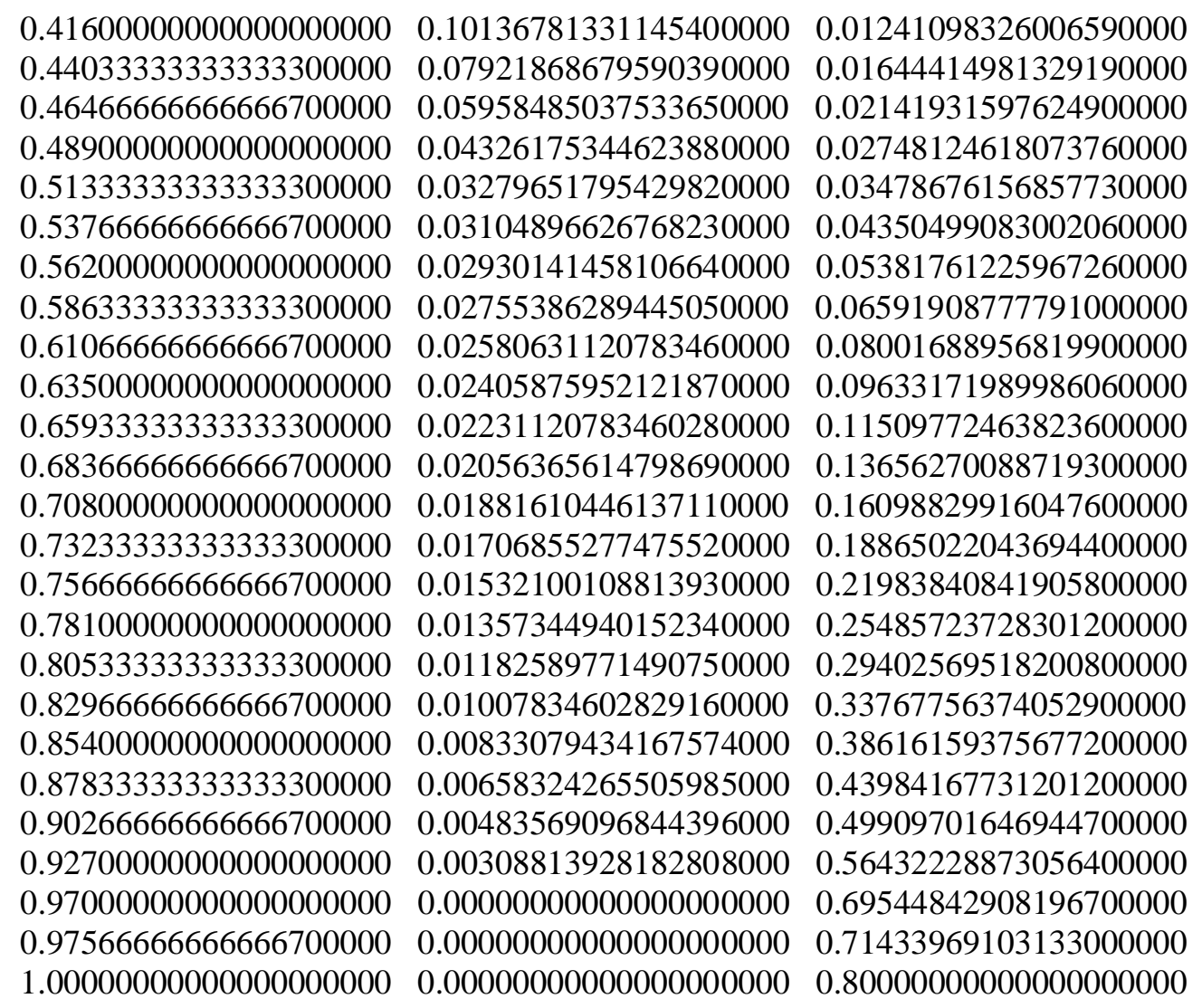

**\$ Test 7

ROCKFLUID

**\$ Injection Stages

RPT 1 WATWET

** S1 $\quad$ krg $\quad$ krog

SLT

$\begin{array}{lll}0.06980 & 1.00000 & 0.00000 \\ 0.10188 & 0.95593 & 0.00062 \\ 0.13395 & 0.91229 & 0.00282 \\ 0.16603 & 0.86911 & 0.00687 \\ 0.19810 & 0.82640 & 0.01292 \\ 0.23018 & 0.78416 & 0.02108 \\ 0.26226 & 0.74242 & 0.03146 \\ 0.29433 & 0.70118 & 0.04413 \\ 0.32641 & 0.66047 & 0.05916 \\ 0.35848 & 0.62031 & 0.07662 \\ 0.39056 & 0.58070 & 0.09656\end{array}$




$\begin{array}{lll}0.42263 & 0.54168 & 0.11904 \\ 0.45471 & 0.50327 & 0.14409 \\ 0.48679 & 0.46549 & 0.17178 \\ 0.51886 & 0.42837 & 0.20213 \\ 0.55094 & 0.39194 & 0.23519 \\ 0.58301 & 0.35624 & 0.27099 \\ 0.61509 & 0.32131 & 0.30957 \\ 0.64717 & 0.28719 & 0.35096 \\ 0.67924 & 0.25394 & 0.39519 \\ 0.71132 & 0.22162 & 0.44230 \\ 0.74339 & 0.19030 & 0.49231 \\ 0.77547 & 0.16008 & 0.54525 \\ 0.80754 & 0.13106 & 0.60114 \\ 0.83962 & 0.10340 & 0.66002 \\ 0.87170 & 0.07727 & 0.72191 \\ 0.90377 & 0.05297 & 0.78683 \\ 0.93585 & 0.03094 & 0.85480 \\ 0.99733 & 0.00000 & 0.99371 \\ 1.00000 & 0.00000 & 1.00000\end{array}$

**\$ Production Stages

RPT 2 WATWET

$\begin{array}{lll}\text { ** S1 } & \text { krg } & k \text { krog } \\ \text { SLT } & & \\ & & \\ 0.06980 & 0.60920 & 0.00000 \\ 0.10188 & 0.56651 & 0.00000 \\ 0.13395 & 0.52537 & 0.00000 \\ 0.16603 & 0.48578 & 0.00000 \\ 0.19810 & 0.44775 & 0.00000 \\ 0.23018 & 0.41127 & 0.00000 \\ 0.26226 & 0.37633 & 0.00000 \\ 0.29433 & 0.34296 & 0.00001 \\ 0.32641 & 0.31113 & 0.00002 \\ 0.35848 & 0.28085 & 0.00005 \\ 0.39056 & 0.25213 & 0.00013 \\ 0.42263 & 0.22496 & 0.00029 \\ 0.45471 & 0.19933 & 0.00060 \\ 0.48679 & 0.17526 & 0.00118 \\ 0.51886 & 0.15274 & 0.00220 \\ 0.55094 & 0.13177 & 0.00393 \\ 0.58301 & 0.11235 & 0.00676 \\ 0.61509 & 0.09447 & 0.01125 \\ 0.64717 & 0.07815 & 0.01819 \\ 0.67924 & 0.06338 & 0.02865 \\ 0.71132 & 0.05015 & 0.04408\end{array}$




$\begin{array}{lll}0.74339 & 0.03847 & 0.06642 \\ 0.77547 & 0.02834 & 0.09818 \\ 0.80754 & 0.01976 & 0.14263 \\ 0.83962 & 0.01272 & 0.20394 \\ 0.87170 & 0.00722 & 0.28738 \\ 0.90377 & 0.00328 & 0.39954 \\ 0.93585 & 0.00087 & 0.54861 \\ 0.97000 & 0.00000 & 0.75925 \\ 1.00000 & 0.00000 & 1.00000 \\ & & \end{array}$

ROCKFLUID

**\$ Injection Stages

RPT 1 WATWET

** S1

$\mathrm{krg}$

krog

SLT

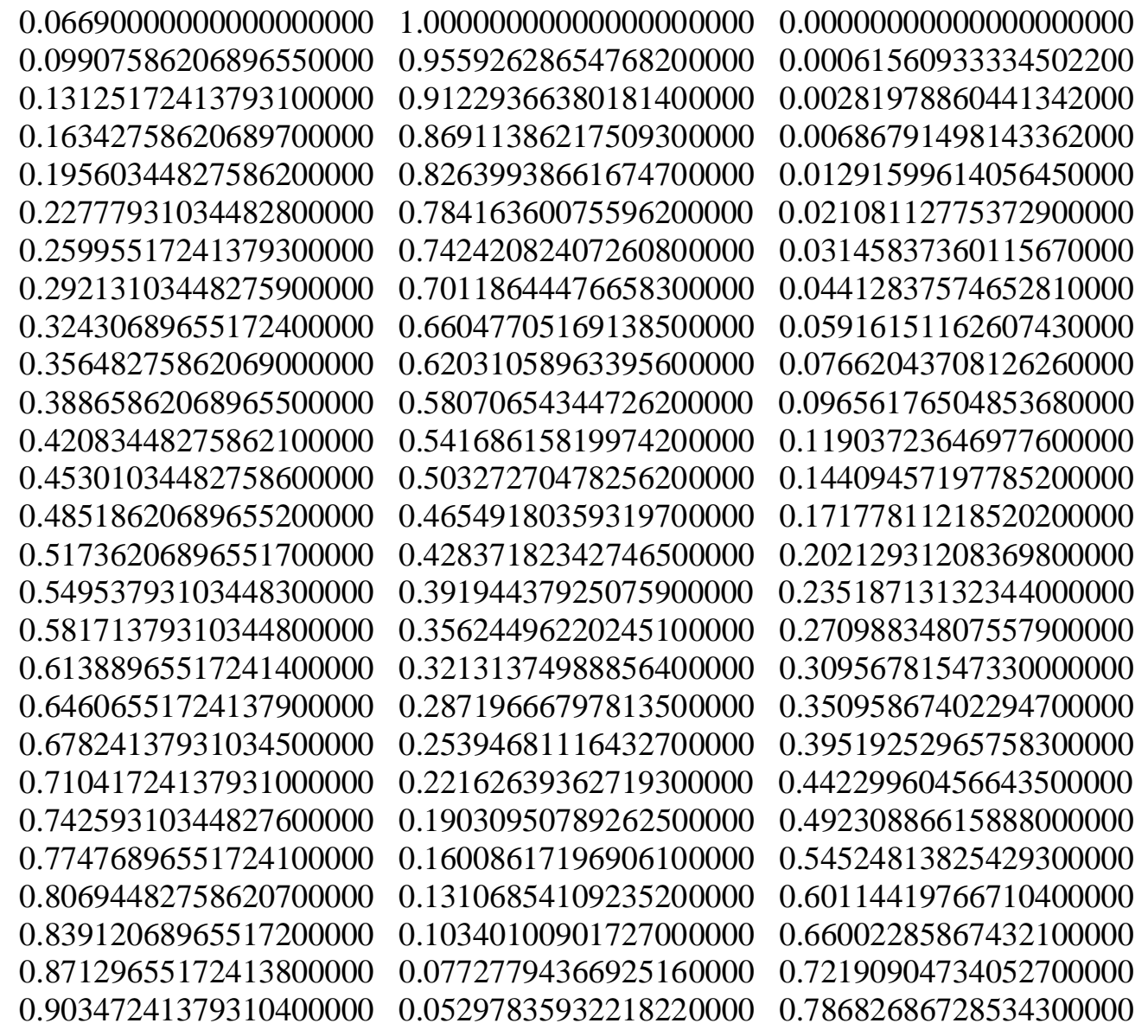


$\begin{array}{lll}0.93564827586206900000 & 0.03094576963317070000\end{array}$ $0.99733000000000000000 \quad 0.00000000000000000000$ $1.00000000000000000000 \quad 0.00000000000000000000$

$* * \$$ Production Stages

\section{RPT 2 WATWET}

** S1

SLT

0.06690000000000000000 0.09907586206896550000

0.13125172413793100000

0.16342758620689700000

0.19560344827586200000

0.22777931034482800000

0.25995517241379300000

0.29213103448275900000

0.32430689655172400000

0.35648275862069000000

0.38865862068965500000

0.42083448275862100000

0.45301034482758600000

0.48518620689655200000

0.51736206896551700000

0.54953793103448300000

0.58171379310344800000

0.61388965517241400000

0.64606551724137900000

0.67824137931034500000

0.71041724137931000000

0.74259310344827600000

0.77476896551724100000

0.80694482758620700000

0.83912068965517200000

0.87129655172413800000

0.90347241379310400000

0.93564827586206900000

0.97000000000000000000

1.00000000000000000000

**\$ Test 9

\section{ROCKFLUID}

**\$ Injection Stages krg $\quad \operatorname{krog}$

0.60920000000000000000

0.56651247264715100000

0.52537721073019400000

0.48579406813691800000

0.44776289305065600000

0.41128352748595100000

0.37635580676505900000

0.34297955892479800000

0.31115460404079500000

0.28088075345304700000

0.25215780887254800000

0.22498556134333300000

0.19936379002688000000

0.17529226076586900000

0.15277072437036000000

0.13179891454983100000

0.11237654538612200000

0.09450330820024240000

0.07817886760189810000

0.06340285640961830000

0.05017486896415470000

0.03849445207481540000

0.02836109232587140000

0.01273306756965740000

0.00723684635030229000

0.00328442922408510000

0.00087425036207513500

0.00000000000000000000

0.00000000000000000000
0.01977419747548630000
0.85479965817781100000

0.99372847452915000000

1.00000000000000000000
0.00000000000000000000

0.00000000000051720560

0.00000000017489070532

0.00000000527469609067

0.00000005913849087524

0.00000038553136155672

0.00000178361432108350

0.00000651259998419494

0.00001999740979178520

0.00005379373060238130

0.00013036566389390800

0.00029035136027271800

0.00060312101241216500

0.00118156684220380000

0.00220220585216933000

0.00393182277155598000

0.00676203251828331000

0.01125329836229750000

0.01819010358844090000

0.02864914061765880000

0.04408255207533560000

0.06641843303356080000

0.09818098245396000000

0.14263287458602800000

0.20394260761043000000

0.28737977704490700000

0.39954141524816900000

0.54861273566786500000

0.75991146243085200000

1.00000000000000000000 
RPT 1 WATWET

$\begin{array}{lll}* * \mathrm{Sl} & \mathrm{krg} & \mathrm{krog} \\ \mathrm{SLT} & & \end{array}$

$0.07250000000000000000 \quad 1.00000000000000000000$ $0.09628205128205130000 \quad 0.96718507419057100000$ $0.12006410256410300000 \quad 0.93461243404397700000$ $\begin{array}{lll}0.14384615384615400000 & 0.90228678693365300000\end{array}$ $0.16762820512820500000 \quad 0.87021306495488500000$ $\begin{array}{lll}0.19141025641025600000 & 0.83839644237977400000\end{array}$ $\begin{array}{lll}0.21519230769230800000 & 0.80684235502142500000\end{array}$ $\begin{array}{lll}0.23897435897435900000 & 0.77555652178040600000\end{array}$ $\begin{array}{lll}0.26275641025641000000 & 0.74454496869536200000\end{array}$ $\begin{array}{lll}0.28653846153846200000 & 0.71381405587904400000\end{array}$ $0.31032051282051300000 \quad 0.68337050779373900000$ $0.33410256410256400000 \quad 0.65322144740966900000$ $0.35788461538461500000 \quad 0.62337443490096600000$ $\begin{array}{lll}0.38166666666666700000 & 0.59383751167263100000\end{array}$ $0.40544871794871800000 \quad 0.56461925068646500000$ $\begin{array}{lll}0.42923076923076900000 & 0.53572881427558800000\end{array}$ $\begin{array}{lll}0.45301282051282000000 & 0.50717602092089300000\end{array}$ $\begin{array}{lll}0.47679487179487200000 & 0.47897142282947900000\end{array}$ $0.50057692307692300000 \quad 0.45112639663395700000$ $\begin{array}{ll}0.52435897435897400000 & 0.42365325016387300000\end{array}$ $0.54814102564102600000 \quad 0.39656534908558200000$ $\begin{array}{lll}0.57192307692307700000 & 0.36987726835154500000\end{array}$ $\begin{array}{ll}0.59570512820512800000 & 0.34360497497284900000\end{array}$ $\begin{array}{lll}0.61948717948717900000 & 0.31776605082495800000\end{array}$ $\begin{array}{lll}0.64326923076923100000 & 0.29237996731791200000\end{array}$ $\begin{array}{lll}0.66705128205128200000 & 0.26746842828666800000\end{array}$ $0.69083333333333300000 \quad 0.24305580416206100000$ $\begin{array}{lll}0.71461538461538500000 & 0.21916969067019700000\end{array}$ $\begin{array}{lll}0.73839743589743600000 & 0.19584164123282000000\end{array}$ $\begin{array}{lll}0.76217948717948700000 & 0.17310814796151200000\end{array}$ $\begin{array}{lll}0.78596153846153800000 & 0.15101198928893500000\end{array}$ $\begin{array}{lll}0.80974358974359000000 & 0.12960413798802800000\end{array}$ $\begin{array}{lll}0.83352564102564100000 & 0.10894656348129800000\end{array}$ $\begin{array}{lll}0.85730769230769200000 & 0.08911653938134150000\end{array}$ $\begin{array}{lll}0.88108974358974400000 & 0.07021366182299910000\end{array}$ $\begin{array}{lll}0.90487179487179500000 & 0.05237220457413860000\end{array}$ $\begin{array}{lll}0.92865384615384600000 & 0.03578536484585640000\end{array}$ $\begin{array}{lll}0.95243589743589700000 & 0.02076144841864090000\end{array}$ $0.99733000000000000000 \quad 0.00000000000000000000$ 1.00000000000000000000 0.00000000000000000000

0.00000000000000000000 0.00032123115206923400 0.00147139410162216000 0.00358374722782670000 0.00673969690779518000 0.01100034484281230000 0.01641529627111790000 0.02302663103396540000 0.03087107278659470000 0.03998131597830690000 0.05038690180968310000 0.06211482922545770000 0.07518999933262240000 0.08963554950945530000 0.10547311138835100000 0.12272301450722900000 0.14140445008631700000 0.16153560484163500000 0.18313377182326900000 0.20621544332550100000 0.23079638959114400000 0.25689172610624100000 0.28451597162005200000 0.31368309854410700000 0.34440657702821600000 0.37669941374399300000 0.41057418620298800000 0.44604307327964200000 0.48311788248703000000 0.52181007445707000000 0.56213078500029800000 0.60409084505888400000 0.64770079881695100000 0.69297092019172000000 0.73991122789586700000 0.78853149923401800000 0.83884128277353700000 0.89084991001071500000 0.99369067395963900000 1.00000000000000000000

**\$ Production Stages 
RPT 2 WATWET

$\begin{array}{lll}* * \mathrm{Sl} & \mathrm{krg} & \mathrm{krog}\end{array}$

$0.07250000000000000000 \quad 0.60920000000000000000$

$0.09628205128205130000 \quad 0.57730369491922000000$

$0.12006410256410300000 \quad 0.54626604203224700000$

$0.14384615384615400000 \quad 0.51608698209375000000$

$0.16762820512820500000 \quad 0.48676645418073000000$

$0.19141025641025600000 \quad 0.45830439559458200000$

$0.21519230769230800000 \quad 0.43070074175433000000$

$\begin{array}{lll}0.23897435897435900000 & 0.40395542607991200000\end{array}$

$\begin{array}{lll}0.26275641025641000000 & 0.37806837986426300000\end{array}$

$\begin{array}{lll}0.28653846153846200000 & 0.35303953213270500000\end{array}$

$\begin{array}{lll}0.31032051282051300000 & 0.32886880948789900000\end{array}$

$0.33410256410256400000 \quad 0.30555613593833500000$

$\begin{array}{lll}0.35788461538461500000 & 0.28310143270790600000\end{array}$

$\begin{array}{lll}0.38166666666666700000 & 0.26150461802371200000\end{array}$

$0.40544871794871800000 \quad 0.24076560687862200000$

$\begin{array}{lll}0.42923076923076900000 & 0.22088431076442600000\end{array}$

$\begin{array}{lll}0.45301282051282000000 & 0.20186063737050700000\end{array}$

$\begin{array}{lll}0.47679487179487200000 & 0.18369449024183000000\end{array}$

$\begin{array}{lll}0.50057692307692300000 & 0.16638576838855200000\end{array}$

$\begin{array}{lll}0.52435897435897400000 & 0.14993436583769400000\end{array}$

$\begin{array}{lll}0.54814102564102600000 & 0.13434017111478000000\end{array}$

$\begin{array}{lll}0.57192307692307700000 & 0.11960306664005900000\end{array}$

$0.59570512820512800000 \quad 0.10572292801941100000$

$\begin{array}{lll}0.61948717948717900000 & 0.09269962320391860000\end{array}$

$\begin{array}{lll}0.64326923076923100000 & 0.08053301148347580000\end{array}$

$0.66705128205128200000 \quad 0.06922294226759330000$

$0.69083333333333300000 \quad 0.05876925358874280000$

$\begin{array}{lll}0.71461538461538500000 & 0.04917177023695440000\end{array}$

$\begin{array}{lll}0.73839743589743600000 & 0.04043030139339440000\end{array}$

$\begin{array}{lll}0.76217948717948700000 & 0.03254463756531700000\end{array}$

$\begin{array}{lll}0.78596153846153800000 & 0.02551454651636960000\end{array}$

$\begin{array}{lll}0.80974358974359000000 & 0.01933976769730620000\end{array}$

$\begin{array}{lll}0.83352564102564100000 & 0.01402000433276050000\end{array}$

$\begin{array}{lll}0.85730769230769200000 & 0.00955491162327301000\end{array}$

$\begin{array}{lll}0.88108974358974400000 & 0.00594407799151932000\end{array}$

$\begin{array}{lll}0.90487179487179500000 & 0.00318699245991945000\end{array}$

$\begin{array}{lll}0.92865384615384600000 & 0.00128297949198453000\end{array}$

$0.97000000000000000000 \quad 0.00000000000000000000$

$\begin{array}{lll}0.97621794871794900000 & 0.00000000000000000000\end{array}$

1.00000000000000000000

0.00000000000000000000

0.00000000000000000000

0.00000000000004292109

0.00000000001451356869

0.00000000043772860245

0.00000000490769676902

0.00000003199390091742

0.00000014801592180002

0.00000054045792219801

0.00000165951518159247

0.00000446415378484765

0.00001081859103968520

0.00002409524510352020

0.00005005090593515600

0.00009805410466254390

0.00018275337069744200

0.00032628823675996900

0.00056115745685838400

0.00093387191989704500

0.00150953315324657000

0.00237749209970747000

0.00365825700298505000

0.00551183373769015000

0.00814769675785422000

0.01183660400193050000

0.01692448457210170000

0.02384864379208730000

0.03315654633189800000

0.04552745446211720000

0.06179721515681710000

0.08298650669619160000

0.11033287262086300000

0.14532688835346800000

0.18975282352379100000

0.24573418100593200000

0.31578451189461500000

0.40286392410793900000

0.51044172100094800000

0.75863191316417700000

0.80393806285791000000

1.00000000000000000000 